\title{
Quantum Clocks are More Accurate Than Classical Ones
}

\author{
Mischa P. Woods $\odot,{ }^{1,2, *, \dagger}$ Ralph Silva,,${ }^{1,3, \dagger}$ Gilles Pütz®,${ }^{1}$ Sandra Stupar, ${ }^{1}$ and Renato Renner® ${ }^{1}$ \\ ${ }^{1}$ Institute for Theoretical Physics, ETH Zurich, Switzerland \\ ${ }^{2}$ Department of Computer Science, University College London, United Kingdom \\ ${ }^{3}$ Département de Physique Appliquée, Université de Genève, Switzerland
}

(Received 28 January 2020; revised 20 September 2021; accepted 21 December 2021; published 3 February 2022)

\begin{abstract}
A clock is, from an information-theoretic perspective, a system that emits information about time. One may therefore ask whether the theory of information imposes any constraints on the maximum precision of clocks. Here we show a quantum-over-classical advantage for clocks or, more precisely, the task of generating information about what time it is. The argument is based on information-theoretic considerations: we analyze how the accuracy of a clock scales with its size, measured in terms of the number of bits that could be stored in it. We find that a quantum clock can achieve a quadratically improved accuracy compared to a purely classical one of the same size.
\end{abstract}

DOI: 10.1103/PRXQuantum.3.010319

\section{INTRODUCTION}

Timekeeping is one of the oldest ways in which humanity has organized its activities, dating back to ancient civilizations that observed the solar cycles. Eventually, we invented our own devices to mark the passage of time, and the advancements in these clocks allowed for revolutionary capabilities such as maritime navigation, and enabled the industrial revolution. The best clocks today are very sophisticated and need a quantum description to understand how they work [1]. The next generation of quantum clocks will enable new applications, such as faster telecommunications, non-satellite-based GPS systems, and also foster advances in fundamental physics, e.g., in the context of gravitational-wave detection [2].

However, quantum theory suggests that there is a limit to the maximum precision of clocks. In contrast to position, momentum, and energy, time cannot be made into an ideal observable [3-5]. Furthermore, a clock must not only evolve with time, but also emit information about its state to the outside world [6], like in the case of a ticking watch, or bell tower. It is thus vulnerable to the disturbance inherent to any quantum measurement [7]; as can be seen in the settings of autonomous quantum control [8] and thermodynamics [9].

\footnotetext{
*mischa.woods@gmail.com

${ }^{\dagger}$ M.W. and R.S. contributed equally to the results.
}

Published by the American Physical Society under the terms of the Creative Commons Attribution 4.0 International license. Further distribution of this work must maintain attribution to the author(s) and the published article's title, journal citation, and DOI.
So we currently find ourselves at an interesting juncture: on the one hand, clocks are increasingly more precise - and just as pendulum clocks enabled the industrial revolution, the next generation of atomic clocks will do the same for a new technological age. However, on the other hand, quantum mechanics suggests that there must be a limit to their increasing accuracy. As an analogy, consider the birth of thermodynamics in the late 18th century: even as heat engines were developed and improved upon, Clausius, Carnot, and others found fundamental limits to their efficiency by relating it to temperature and heat. In the case of clocks a natural question is thus as follows: can we relate their precision to physical variables such as entropy, energy, size, or information contents, and by doing so, quantify the fundamental limits to their accuracy?

We investigate the effect of the size of a clock, motivated by the general observation that the disturbance suffered by large mechanical clocks by the act of reading-off time appears insignificant, while tiny clocks are more prone to be disturbed. There are a number of ways to quantify the size of a clock, e.g., by its mass [10]. We take an information-theoretic approach, and consider the size of the state space of the clock, which is the number of perfectly distinguishable states that it can be in, or alternatively, the dimension $d$ of its associated Hilbert space. Indeed, a clock of size $d$ is a clock that could in principle store at $\operatorname{most} \log _{2} d$ bits of information in its internal state, and thus $d$ is a measure of its information contents.

Moreover, it is interesting to ask whether quantum features in clocks could provide an advantage. In order to make a comparison, one can introduce the notion of a classical clock as a quantum clock that has lost its quantum properties through decoherence. 
This paper proves a fundamental connection between the size of a quantum or classical clock and its attainable accuracy. Namely we find that there exist quantummechanical clocks based on Ref. [8], whose accuracy represents a quadratic improvement over the best classical clocks of the same size.

To clarify what is meant by "a clock" in this work, we distinguish between two types of devices for measuring time: timepieces that output time information on request, like a stopwatch, and clocks that output time information autonomously, like a chiming clock. They serve different purposes. Stop watches are used to measure a time interval between events triggered by external processes (e.g., between the event that a train leaves the station at $A$ and the event that it arrives at $B$ ) [11]. Conversely, chiming clocks "generate" events themselves, which may then be used to trigger external events (e.g., that the train leaves the station at $A$ ), see Fig. 1.

This work is concerned with the second type of timekeeping. Hence, from now on (and with the exception of the review of earlier work at the beginning of Sec. II) we use the term clock for devices that output information about time autonomously [12]. Specifically, we take a clock to be a device that generates a sequence of individual events, which we call ticks. For the purpose of this discussion, we assume that the ticks are the only information output by the clock.

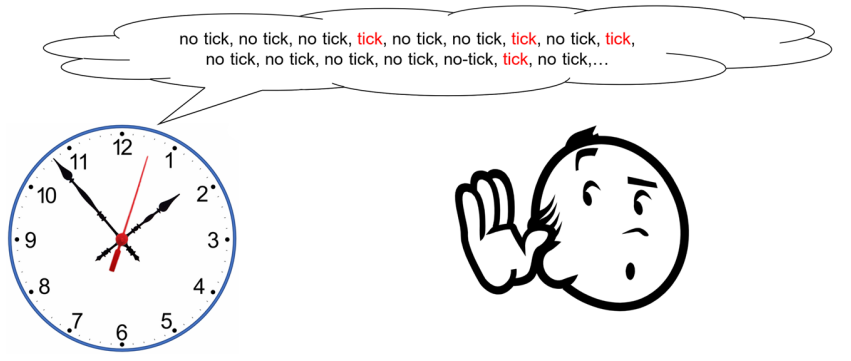

FIG. 1. Illustration of a (chiming) clock. A (chiming) clock produces a continuous stream of "no-tick", "tick" information. The "no ticks" represent the silence between ticks. Note that the silence between ticks is just as important as the ticks themselves for the functioning of a clock. To illustrate how (chiming) clocks and stopwatches serve different purposes, consider two examples: case (a) two people agree to meet at a given time in the future, say $38 \mathrm{~h}$ from now. If one has a (chiming) clock, one can simply count the emitted ticks until the time interval of $38 \mathrm{~h}$ has passed. However, in contrast, if one has a stopwatch there is no external trigger one can use and measuring the stopwatch at a random (unknown) time, would result in a measure zero probability of getting (even approximately) the outcome of $38 \mathrm{~h}$ later. Case (b) a race takes place and one wants to know the time of the winner. Here there is an external trigger (the winner crossing the finish line), and a stopwatch will suffice. In case (b) a (chiming) clock will also suffice of course. So a (chiming) clock may be used to replace a stopwatch but not vice versa.
The accuracy of a clock is then defined via the regularity of its ticks. We ask the simple operational question: how many ticks can a clock output before the uncertainty in its next tick has grown to be as large as the interval between ticks? This accuracy measure, introduced in Ref. [9], is referred to as $R$ (Sec. II E).

We use the term quantum clock for a clock whose dynamics is not subject to any constraints other than those imposed by quantum theory. Their internal state can therefore be represented by a density operator in a $d$-dimensional Hilbert space, and the transition from the clock's state at a time $t_{1}$ to its state at time $t_{2}$ corresponds to a trace-preserving completely positive map. We also consider the special case of classical clocks, where decoherence is assumed to occur on a timescale that is much shorter than the processes responsible for the generation of ticks. Their state space is therefore restricted to a fixed set consisting of $d$ perfectly distinguishable states and their probabilistic mixtures - the "classical" states. In this case, a state transition from time $t_{1}$ to $t_{2}$ is most generally represented by a stochastic map.

Our main results are bounds on the accuracy, which depends on the clock's size $d$. On the one hand, we prove that, for any fixed $\eta>0$, there exist quantum clocks whose accuracy scales as

$$
R_{\text {quantum }} \geq \Omega\left(d^{2-\eta}\right) .
$$

That is, quantum clocks can have an accuracy that grows essentially quadratically in the clock's size for large $d$. We prove this statement by construction, showing that the socalled quasi-ideal clocks proposed in Ref. [8] can achieve this scaling under the appropriate circumstances. On the other hand, we prove that the accuracy of any classical clock is upper bounded by

$$
R_{\text {classical }} \leq d,
$$

and show that a simple stochastic clock, studied in Ref. [13] in the context of the alternate ticks game, saturates this bound. Combining Eqs. (1) and (2), we conclude that for large size $d$, quantum clocks outperform classical ones quadratically in terms of their accuracy $R$.

\section{MODELING CLOCKS}

To motivate our framework for describing clocks, we first have a look at existing models that have been considered in the literature and discuss their features and limitations. (An extensive review on prior literature regarding clocks and the general issue of time in quantum mechanics can be found in Refs. [14,15].)

Pauli regarded an "ideal clock" as a device that has an observable $T$ whose value is in one-to-one correspondence to the time parameter $t$ in the quantum-mechanical 
equation of motion. The observable $T$ would need to satisfy $(d / d t) T=\mathbb{1}$. Furthermore, since neither $T$ nor the Hamiltonian of the system, $H$, should depend on time explicitly, they would need to satisfy the commutation relation $i[H, T]=\mathbb{1}[16]$. Pauli then argued that this implies that $H$ has as its spectrum the full real line [17]. Since such Hamiltonians are unphysical, he concluded that an observable $T$ with the desired properties, and hence an ideal clock, cannot exist $[3,5,18]$. As such, these objects are referred to as idealized clocks.

This raises the question whether one can at least approximate an idealized clock. Salecker and Wigner [10] and Peres [19] considered finite-dimensional constructions. Specifically, they showed that for any dimension $d$ and for any fixed time interval $\Delta$ there exists a clock, which we refer to as the SWP clock, whose Hamiltonian satisfies

$$
\forall k \in\{0, \ldots, d-1\}: \quad e^{i H \Delta}\left|\theta_{k}\right\rangle=\left|\theta_{k+1}(\bmod d)\right\rangle,
$$

where $\left\{\left|\theta_{i}\right\rangle\right\}_{i=0}^{d-1}$ is the SWP basis-an orthonormal basis of the clock's Hilbert space. Hence, if the clock was initialized to state $\left|\theta_{0}\right\rangle$ and if one did read the clock at a time $t \in\{0, \Delta, 2 \Delta, \ldots\}$ by applying a projective measurement with respect to the SWP basis, the outcome would be precise information about time $n(\bmod d)$. However, in between these particular points in time, the amplitudes of the basis states are in general all nonzero [20]. Hence, if the clock was measured, say, at $t=5 / 2 \Delta$, the outcome would be uncertain [21]. In addition, such a measurement would disturb the clock's state, effectively resetting it to a random time. This problem was resolved in recent work by some of us, with the introduction of the so-called quasi-ideal clock [8], which is able to approximate the dynamical behavior of Pauli's idealized clock while maintaining a finite dimension.

The constructions from Refs. [10,19], however, do not include a mechanism to output time information autonomously. Hence, to use the terminology introduced earlier, they are stopwatches rather than chiming clocks. To extract time information from them, one would have to apply measurements from the outside. But then the outcome depends on when and how these measurements are performed. Thus, in order to reasonably talk about their accuracy - in terms of operationally motivated quantities - we need a more complete description. In Ref. [8], a potential term was added to the Hamiltonian. In the case that this potential is pure imaginary, it will allow us to model information about time being extracted autonomously. This feature, together with the definition of quantum clocks as outlined in the following section, will allow for the accuracy of quantum clocks to be bounded.

\section{A. Quantum clocks}

The modeling of clocks that we use here follows the operational approach introduced in Ref. [6] with some adjustments. We now explain this setup in detail.

A $d$-dimensional quantum clock consists of a (generally open) quantum system $C$, which we call the clockwork. The transition of a clockwork's state $\rho_{C, t}$ at some time $t$ to its state $\rho_{C, t+\Delta}$ at a later time $t+\Delta$ can hence most generally be described by a trace-preserving completely positive map

$$
\mathcal{M}_{C \rightarrow C}^{\Delta}: \quad \rho_{C, t} \mapsto \rho_{C, t+\Delta},
$$

which depends on $\Delta \in \mathbb{R}_{\geq 0}$ but not on $t \in \mathbb{R}$. Note that these maps form a family parameterized by $\Delta \in \mathbb{R}_{\geq 0}$. For the particular choice $\Delta=0$ it is the identity map,

$$
\mathcal{M}_{C \rightarrow C}^{(0)}=\mathcal{I}_{C}
$$

Furthermore, the maps are mutually commutative under composition, i.e.,

$$
\mathcal{M}_{C \rightarrow C}^{\Delta+\Delta^{\prime}}=\mathcal{M}_{C \rightarrow C}^{\Delta^{\prime}} \circ \mathcal{M}_{C \rightarrow C}^{\Delta}=\mathcal{M}_{C \rightarrow C}^{\Delta} \circ \mathcal{M}_{C \rightarrow C}^{\Delta^{\prime}},
$$

for any $\Delta, \Delta^{\prime} \in \mathbb{R}_{\geq 0}$. In other words, the evolution of $C$ is determined by a one-parameter family of maps, $\left\{\mathcal{M}_{C \rightarrow C}^{\Delta}\right\}_{\Delta \in \mathbb{R}_{\geq 0}}$, and which are Markovian. The Markovianity assumption is necessary, otherwise the generators of the dynamics could change at regular intervals, providing an unaccounted timing resource for the clock.

Assuming that the energy that drives the clockwork's evolution is finite, we may additionally assume that the clockwork's state changes at a finite speed. This means that the function $\Delta \mapsto \mathcal{M}_{C \rightarrow C}^{\Delta}$ is continuous. But, using Eqs. (3) and (4), this is in turn equivalent to the requirement that

$$
\lim _{\Delta \rightarrow 0} \mathcal{M}_{C \rightarrow C}^{\Delta}=\mathcal{I}_{C}
$$

which may be regarded as a strengthening of Eq. (3).

Since we assume that the clockwork's evolution is time independent, its description in terms of the entire family $\mathcal{M}_{C \rightarrow C}^{\Delta}$, for $\Delta \in \mathbb{R}_{\geq 0}$, is highly redundant. Indeed, using Eq. (4) we may write

$$
\mathcal{M}_{C \rightarrow C}^{\Delta}=\lim _{\delta \rightarrow 0}\left(\mathcal{M}_{C \rightarrow C}^{\delta}\right)^{\left\lfloor\frac{\Delta}{\delta}\right\rfloor},
$$

where we use the notation

$$
\left(\mathcal{M}_{C \rightarrow C}^{\delta}\right)^{k}=\underbrace{\mathcal{M}_{C \rightarrow C}^{\delta} \circ \cdots \circ \mathcal{M}_{C \rightarrow C}^{\delta}}_{k \text { times }} .
$$

It thus suffices to specify the evolution map for arbitrarily small time parameters, which we in the following denote 
by $\delta$. (The evolution is thus governed by the Lindblad equation, a fact that we exploit in Sec. II B.)

The maps $\mathcal{M}_{C \rightarrow C}^{\delta}$ describe the evolution of the state on $C$. But, as argued above, we are generally interested in the information that the clock transmits to the outside. This can be included in our description by virtue of extensions of the maps $\mathcal{M}_{C \rightarrow C}^{\delta}$. That is, we consider maps $\mathcal{M}_{C \rightarrow C T_{i}}^{\delta}$ whose target space is a composite system, consisting of $C$ and an additional system $T_{i}$, such that

$$
\mathcal{M}_{C \rightarrow C}^{\delta}=\operatorname{tr}_{T_{i}} \circ \mathcal{M}_{C \rightarrow C T_{i}}^{\delta} .
$$

We call $T_{i}$ tick registers, alluding to the idea that the basic elements of information emitted by a clock are its "ticks," see Fig. 2. Note that while the model of a clock considered here involves an unbounded sequence of finitedimensional registers that carry the time information it generates, one can show, see [22], that one is able to achieve the precision as reported here for quantum and classical clocks with a single finite register attached to the clock. The model in [22] can achieve this by only utilising a new register state when the clock ticks, in contrast to requiring a new qubit register, $T_{i}$, for every infinitesimal time step. Therefore, its register only needs to be as large as the number of ticks one wishes to record

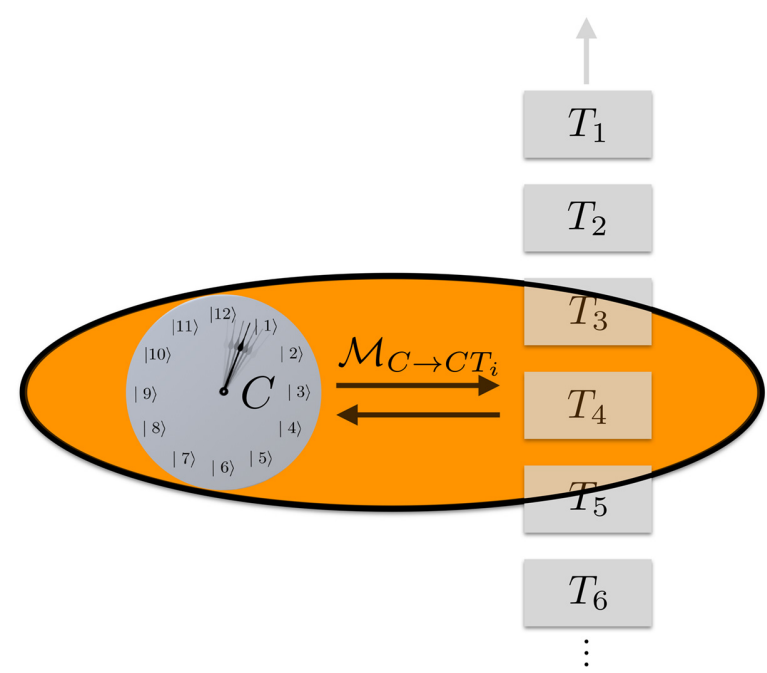

FIG. 2. Information-theoretic clock model. A clock is modeled as a device that emits information about time. This flow of information is described by small discrete time steps $\delta$, specified by maps $\mathcal{M}_{C \rightarrow C T_{i}}^{\delta}$ [6]. In each time step $\delta$ the clockwork $C$ interacts with a tick register $T_{i}, i \in \mathbb{N}$. The latter models the part of the information that the clock emits to the outside and hence is unavailable to the clock at any later step. Since we are considering the case in which time is continuous, in our analysis we take the continuous limit in which each time step is infinitesimal. Conversely, $C$ must include all information that remains accessible to the clock over more than one time step. The emission of information into the registers $\left\{T_{i}\right\}_{i}$ induces quantum back action on $C$, and thus a degradation of the information it contains. with it. Furthermore, this alternative model has a master equation description for the entire register and clockwork; and hence, contrary to the model considered here, does not require additional degrees of freedom to account for the alignment of the clockwork with a new register $T_{i}$ at every infinitesimal time step.

After these general remarks, we are now ready to state the technical definition. In the following we let all the tick registers $T_{i}$ be isomorphic to a single-tick register denoted $T$.

Definition 1: (From Ref. [6].) A (quantum) clock is a pair $\left(\rho_{C}^{0},\left\{\mathcal{M}_{C \rightarrow C T}^{\delta}\right\}_{\delta}\right)$, consisting of a density operator $\rho_{C}^{0}$ on a d-dimensional Hilbert space $C$ together with a family of trace-preserving and completely positive maps $\mathcal{M}_{C \rightarrow C T}^{\delta}$ from $C$ to $C \otimes T$, where $T$ is an arbitrary system, such that the following limits exist and take on the value

$$
\lim _{\Delta \rightarrow 0} \lim _{\delta \rightarrow 0}\left(\operatorname{tr}_{T} \circ \mathcal{M}_{C \rightarrow C T}^{\delta}\right)^{\left\lfloor\frac{\Delta}{\delta}\right\rfloor}=\mathcal{I}_{C} .
$$

Using Eq. (6), it is easy to see that any family of maps whose reduction to $C$ satisfies Eqs. (4) and (5) also satisfies Eq. (9). The converse, however, is not necessarily true. Nevertheless, given a family of maps $\left\{\mathcal{M}_{C \rightarrow C T}^{\delta}\right\}_{\delta}$ as in Definition 1, one may always define a family of maps

$$
\left\{\overline{\mathcal{M}}_{C \rightarrow C}^{\Delta}\right\}_{\Delta}=\left\{\lim _{\delta \rightarrow 0}\left(\operatorname{tr}_{T} \circ \mathcal{M}_{C \rightarrow C T}^{\delta}\right)^{\left\lfloor\frac{\Delta}{\delta}\right\rfloor}\right\}_{\Delta},
$$

which meet both Eqs. (4) and (5). In this sense, specifying a map that satisfies Eq. (9) is indeed sufficient to define the continuous and time-independent evolution of a clock.

The definition does not yet impose any constraints on the tick register, $T$. Since we want to compare different clocks, it will, however, be convenient to assume that $T$ contains two designated orthogonal states, $|1\rangle$ and $|0\rangle$, which we interpret as "tick" and "no tick", respectively. The idea is that ticks are the most basic units of time information that a clock can emit. Roughly speaking, a tick indicates that a certain time interval has passed since the last tick.

To know if the clock has ticked after the application of the map $\mathcal{M}_{C \rightarrow C T}^{\delta}$, one has to measure the tick register in the "tick" basis $\{|0\rangle,|1\rangle\}$. In general, this represents an additional map on the clockwork and register, as Definition 1 allows for the tick register to be coherent in the tick basis, and even entangled with the clockwork system $C$. However, in this work we are only concerned with the probability distribution of ticks (as we characterize the performance of the clock from this alone), and so we incorporate the additional measurement into the map $\mathcal{M}_{C \rightarrow C T}^{\delta}$ itself. This is equivalent to requiring the map to restrict the state of the clockwork and tick register to be block-diagonal states in the basis $\{|0\rangle,|1\rangle\}$.

Furthermore, we consider the behavior of the tick register in the limit $\delta \rightarrow 0$. In principle, the probability of a 
tick in this limit need not be zero. However, such a clock would correspond to one that has a probability of ticking on every application of the map $\mathcal{M}_{C \rightarrow C T}^{\delta}$ independently of the state of the clockwork system, and thus does not provide any information about time [23].

Following the above considerations, we continue with clocks whose maps $\mathcal{M}_{C \rightarrow C T}^{\delta}$ provide states on $C T$ that are diagonal in the tick basis $\{|0\rangle,|1\rangle\}$, and also satisfy the limit

$$
\operatorname{tr}_{C}\left[\lim _{\delta \rightarrow 0} \mathcal{M}_{C \rightarrow C T}^{\delta}\left(\rho_{C}\right)\right]=|0\rangle\left\langle\left. 0\right|_{T} .\right.
$$

One may feel inclined to think of a clock whose "ticks" convey additional information, such as the number of previous ticks produced by the clock. For example, often a church bell will produce different chimes to specify the passing of different hours. To treat this within our model, one may think of a (classical) counter, which merely counts the number of tick registers in the state $|1\rangle$. This way, if a tick occurs, one can read the counter and discern the time. Clearly the counter does not need any additional timing devices to function. Importantly, since such a counter interacts only with the tick registers and not the clockwork, it does not directly affect the evolution of the clockwork system $C$.

This concludes our discussion of the generic model of clocks. Real life clocks may also be subject to additional constraints, such as unavoidable decoherence or power constraints [9,24]. Since we are considering finitedimensional maps from the clockwork to itself, which are continuous, this naturally leads to a finite power consumption, and decoherence is addressed later with our classical clock case. We furthermore comment on aspects of the clock model in the conclusions, Sec. V.

\section{B. Representation in terms of generators}

As explained above, the specification of the individual maps of the family $\left\{\mathcal{M}_{C \rightarrow C T}^{\delta}\right\}_{\delta}$ is redundant. The following lemma, which is basically a variant of the Lindblad representation theorem [25], asserts that the family can equivalently be specified in terms of generators.

Lemma 1: Let $\left(\rho_{C}^{0},\left\{\mathcal{M}_{C \rightarrow C T}^{\delta}\right\}_{\delta}\right)$ be a clock with a classical tick register, having as a basis the states $\{|0\rangle,|1\rangle\}$. Then there exists a Hermitian operator $H$ as well as two families of orthogonal operators $\left\{L_{j}\right\}_{j=1}^{m}$ and $\left\{J_{j}\right\}_{j=1}^{m}$ on C such that

$$
\begin{aligned}
& \mathcal{M}_{C \rightarrow C T}^{\delta}\left(\rho_{C}\right)=\rho_{C} \otimes|0\rangle\left\langle\left. 0\right|_{T}\right. \\
& -\delta\left(i[H, \rho]+\sum_{j=1}^{m} \frac{1}{2}\left\{L_{j}^{\dagger} L_{j}+J_{j}^{\dagger} J_{j}, \rho\right\}-L_{j} \rho L_{j}^{\dagger}\right) \\
& \otimes|0\rangle\left\langle\left. 0\right|_{T}+\delta \sum_{j=1}^{m} J_{j} \rho J_{j}^{\dagger} \otimes \mid 1\right\rangle\left\langle\left. 1\right|_{T}+F_{C \rightarrow C T}^{\delta}\left(\rho_{C}\right),\right.
\end{aligned}
$$

for $\delta>0$, and where $F_{C \rightarrow C T}^{\delta}=O\left(\delta^{2}\right)$. Conversely, given any Hermitian operator $H$ and orthogonal families of operators $\left\{L_{j}\right\}_{j=1}^{m}$ and $\left\{J_{j}\right\}_{j=1}^{m}$ on C, Eq. (12) defines a clock $\left(\rho_{C}^{0},\left\{\mathcal{M}_{C \rightarrow C T}^{\delta}\right\}\right)$ with a classical tick register.

In the case of classical clocks with basis $\left\{\left|c_{n}\right\rangle\right\}, H$ is the zero operator and the operators $L_{j}$ and $J_{j}$ can all be chosen to be proportional to operators of the form $\left|c_{m}\right\rangle\left\langle c_{n}\right|$.

The proof of this Lemma, which is provided in Appendix A 1, follows the description in Sec. 3.5.2 of Ref. [26]. We call $\rho_{C}^{0}$ the initial state of the clockwork. Furthermore, the operators $J_{j}$ are called tick generators [27].

In addition to determining when the clock ticks, the tick generators $J_{j}$ also define the clockwork's state after a tick. Clocks for which this state coincides with the initial state $\rho_{C}^{0}$ are of special interest, for they have a particularly appealing mathematical structure and are optimal in terms of their accuracy in the case of classical clocks.

Definition 2: $A$ reset clock is a quantum clock $\left(\rho_{C}^{0},\left\{\mathcal{M}_{C \rightarrow C T}^{\delta}\right\}_{\delta}\right)$ whose tick generators induce a mapping to the clock's initial state [28], i.e.,

$$
\sum_{j=1}^{m} J_{j} \rho J_{j}^{\dagger} \propto \rho_{C}^{0} \quad \forall \rho \in \mathcal{S}\left(\mathcal{H}_{C}\right)
$$

One may also use the Lindbladian generators to describe the evolution of the clockwork system $C$ as continuous, parametrized by a real variable $t$. From Lemma 1, the following differential equation governs the evolution of the clockwork:

$$
\begin{aligned}
\frac{d}{d t} \rho_{C}(t)= & \lim _{\delta \rightarrow 0} \frac{\operatorname{tr}_{T}\left[\mathcal{M}_{C \rightarrow C T}^{\delta}\left[\rho_{C}(t)\right]\right]-\rho_{C}(t)}{\delta} \\
= & -i\left[H, \rho_{C}(t)\right]+\sum_{j=1}^{m} L_{j} \rho_{C}(t) L_{j}^{\dagger}+J_{j} \rho_{C}(t) J_{j}^{\dagger} \\
& -\frac{1}{2}\left\{L_{j}^{\dagger} L_{j}+J_{j}^{\dagger} J_{j}, \rho_{C}(t)\right\} .
\end{aligned}
$$

For the tick register, one may take the same limit to find the probability density of a tick being recorded, via the probability that the register is in the state $|1\rangle$,

$$
\begin{aligned}
P(t) & =\lim _{\delta \rightarrow 0} \frac{\operatorname{tr}_{C T}\left[|1\rangle\left\langle\left. 1\right|_{T} \mathcal{M}_{C \rightarrow C T}^{\delta}\left[\rho_{C}(t)\right]\right]\right.}{\delta} \\
& =\operatorname{tr}_{C}\left[\sum_{j=1}^{m} J_{j} \rho_{C}(t) J_{j}^{\dagger}\right] .
\end{aligned}
$$

This limit and the sequence of ticks is discussed in more detail in Sec. II E. 
Furthermore, consider the case of a clock in which one focuses on a single tick, and tracks the state of the clock only up to the first tick. In this case one can remove the "tick" channel $\sum_{j=1}^{m} J_{j} \rho_{C} J_{j}^{\dagger}$ from the Lindbladian of the clock in Eq. (14), as it represents the state of the clockwork after a tick (see Lemma 1). Thus the description of the entire family of tick generators $\left\{J_{j}\right\}_{j=1}^{m}$ is redundant. Labeling the state of the clockwork for just a single tick as $\rho_{C}^{(1)}$, its dynamics are given by [taking Eq. (14) with the tick channel removed]

$$
\begin{aligned}
\frac{d}{d t} \rho_{C}^{(1)}(t)= & -i\left[H, \rho_{C}^{(1)}(t)\right]-\left\{V, \rho_{C}^{(1)}(t)\right\} \\
& +\sum_{j=1}^{m} L_{j} \rho_{C}^{(1)}(t) L_{j}^{\dagger}-\frac{1}{2}\left\{L_{j}^{\dagger} L_{j}, \rho_{C}^{(1)}(t)\right\},
\end{aligned}
$$

where

$$
V=\frac{1}{2} \sum_{j=1}^{m} J_{j}^{\dagger} J_{j}
$$

is an arbitrary positive operator representing the ticking dynamics of the clockwork. In this case, the probability density of the first tick being recorded is, from Eq. (15),

$$
P^{(1)}(t)=\operatorname{tr}_{C}\left[2 V \rho_{C}^{(1)}(t)\right] .
$$

This proves useful in the case of reset clocks. As we discuss later, the ticks of a reset clock are a sequence of independent and identically distributed random variables, and thus the first tick suffices to characterize such a clock.

\section{Example}

When describing a clock, one may want to distinguish between the intrinsic evolution of the state of the clockwork and the mechanism that transfers information about this state to the outside. A rather generic way to do this is to describe the evolution of the clockwork by a Hamiltonian $\hat{H}_{C}$ on the system $C$, and the transfer of information to the outside by a continuous measurement of the system's state with respect to a fixed basis $\left\{\left|t_{i}\right\rangle\right\}_{i=0}^{d-1}$, which we refer to as the time basis. In order to ensure that the measurement does not disturb the clock's state too much, the coupling between clockwork and measurement mechanism must be weak. We quantify it in the following by assigning a coupling parameter $V_{i} \in \mathbb{R}_{\geq 0}$ to each of the elements $\left|t_{i}\right\rangle$ of the time basis and consider a reset clock (Definition 2). We could then define a quantum clock $\left(\left|\psi_{0}\right\rangle\left\langle\psi_{0}\right|,\left\{\mathcal{M}_{C \rightarrow C T}^{\delta}\right\}_{\delta}\right)$ with initial state $\left|\psi_{0}\right\rangle\left\langle\psi_{0}\right|$ and maps

$$
\begin{aligned}
\mathcal{M}_{C \rightarrow C T}^{\delta}: & \rho_{C} \mapsto e^{-i \delta \hat{H}_{C}} \hat{M}_{0}^{\delta} \rho_{C} \hat{M}_{0}^{\delta \dagger} e^{i \delta \hat{H}_{C}} \otimes|0\rangle\langle 0| \\
& +\sum_{j=0}^{d-1} e^{-i \delta \hat{H}_{C}} \hat{M}_{1, j}^{\delta} \rho_{C} \hat{M}_{1, j}^{\delta} e^{i \delta \hat{H}_{C}} \otimes|1\rangle\langle 1|,
\end{aligned}
$$

where $\hat{M}_{1, j}^{\delta}=\sqrt{2 \delta V_{j}}\left|\psi_{0}\right\rangle\left\langle t_{j}\right|$ and $\hat{M}_{0}^{\delta}=\sqrt{\mathbb{1}_{C}-2 \delta \hat{V}_{C}}$ with $\hat{V}_{C}=\sum_{i=0}^{d-1} V_{i}\left|t_{i}\right\rangle\left\langle t_{i}\right|$.

For sufficiently small $\delta$, the quantities $\left\{\hat{M}_{1, i}^{\delta} \dagger \hat{M}_{1, i}^{\delta}\right\}_{i=0}^{d-1}$ together with $\hat{M}_{0}^{\delta \dagger} \hat{M}_{0}^{\delta}$ form a positive-operator valued measure (POVM), since

$$
\hat{M}_{0}^{\delta \dagger} \hat{M}_{0}^{\delta}+\sum_{i=0}^{d-1} \hat{M}_{i, 1}^{\delta \dagger} \hat{M}_{i, 1}^{\delta}=\left|\mathbb{1}_{C}-2 \delta \hat{V}_{C}\right|+2 \delta \hat{V}_{C} .
$$

As such, one can interpret Eq. (19), in the following light. The initial state of the clockwork $\rho_{C}$ is measured via the POVMs, followed by allowing the clockwork to freely evolve according to its internal Hamiltonian $\hat{H}_{C}$ for an infinitesimal time step $\delta$ and repeating the process. In accordance with Eq. (19) one would then associate the outcome "no tick" with the POVM element $\hat{M}_{0}^{\delta \dagger} \hat{M}_{0}^{\delta}$ and the "tick" outcome with the elements $\left\{\hat{M}_{1, i}^{\delta} \dagger^{\dagger} \hat{M}_{1, i}^{\delta}\right\}_{i=0}^{d-1}$. Since the POVM defines a measurement with classical outcome, one may regard the tick as a classical value, i.e., the tick register could be assumed to be classical in this case.

Furthermore, by expanding in $\delta$, Eq. (19) can be written in the form

$$
\begin{aligned}
\mathcal{M}_{C \rightarrow C T}^{\delta}: \quad \rho_{C} & \mapsto \rho_{C} \otimes|0\rangle\langle 0| \\
& -\delta\left(i\left[\hat{H}_{C}, \rho\right]+\sum_{j=1}^{m} \frac{1}{2}\left\{J_{j}^{\dagger} J_{j}, \rho\right\}\right) \otimes|0\rangle\langle 0| \\
& +\delta \sum_{j=1}^{m} J_{j} \rho J_{j}^{\dagger} \otimes|1\rangle\langle 1|+O\left(\delta^{2}\right),
\end{aligned}
$$

where we define $J_{j}=\sqrt{2 V_{j}}\left|\psi_{0}\right\rangle\left\langle t_{j}\right|$. With the further identifications $H=\hat{H}_{C}$, and $\left\{L_{j}\right\}_{j=1}^{m}$ with the set of zero operators, we see that Eq. (20) is in the form prescribed by Lemma 1. This ensures that the map $\mathcal{M}_{C \rightarrow C T}^{\delta}$ is indeed a clock, according to our definition, Eq. (9). Consequently, it is easily verified that the $J_{j}$ operators satisfy Eq. (13) and the clock is thus a reset clock. It also follows from Sec. B that in the continuous limit of clocks, the probability of not getting a "tick" in the time interval $[0, t]$ followed by a tick in the interval time $t, t+\delta t$ is

$$
\delta t P^{(1)}(t)=\delta t \operatorname{tr}_{C}\left[2 V \rho_{C}^{(1)}(t)\right]=\delta t \operatorname{tr}_{C}\left[2 \hat{V}_{C} \rho_{C}^{(1)}(t)\right],
$$

where $\rho_{C}^{(1)}(t)=\left|\bar{\psi}_{t}\right\rangle\left\langle\bar{\psi}_{t}\right|$, with

$$
\left|\bar{\psi}_{t}\right\rangle=e^{-i t \hat{H}_{C}-t \hat{V}_{C}}\left|\psi_{0}\right\rangle .
$$

\section{Classical clocks as a special case}

Classical physics is widely believed to arise from quantum mechanics due to a mechanism called decoherence. It 
is a naturally occurring process caused by phenomena in which the quantum state becomes incoherent in some preferred basis [29-31]. Roughly speaking, a classical clock may be regarded as a clock that satisfies Definition 1, but whose state space is restricted to classical states due to decoherence effects, which happen on a timescale much shorter than the times between ticks.

We allow for any preferred basis. Let us denote it by an arbitrary fixed orthonormal basis $\left\{\left|c_{k}\right\rangle\right\}_{k=0}^{d-1}$, of the Hilbert space $C$ of the clockwork:

$$
\mathcal{C}_{\left\{\left|c_{k}\right\rangle\right\}}=\left\{\rho_{C}=\sum_{i=0}^{d-1} p_{i}\left|c_{i}\right\rangle\left\langle c_{i}\right|: p_{i} \geq 0, \sum_{i=0}^{d-1} p_{i}=1\right\} .
$$

Definition 3: A clock $\left(\rho_{C}^{0},\left\{\mathcal{M}_{C \rightarrow C T}^{\delta}\right\}_{\delta}\right)$ is called classical if there exists a basis $\left\{\left|c_{k}\right\rangle\right\}_{k}$ (called the classical basis) such that

$$
\rho_{C}^{0} \in \mathcal{C}_{\left\{\left|c_{k}\right\rangle\right\}} \text { and } \operatorname{tr}_{T} \circ \mathcal{M}_{C \rightarrow C T}^{\delta}\left(\mathcal{C}_{\left\{\left|c_{k}\right\rangle\right\}}\right) \subseteq \mathcal{C}_{\left\{\left|c_{k}\right\rangle\right\}}
$$

for all $\delta \geq 0$.

Since the dynamics is restricted to a single basis, we require only the vector of diagonal elements in that basis to describe the clock, and we label this by

$$
v_{C}=\sum_{m} v_{C, m} \mathbf{e}_{m}
$$

where $\mathbf{e}_{m}$ represents the basis vector $\left|c_{m}\right\rangle_{C}\left\langle c_{m}\right|$ and $v_{C, m}=$ $\left\langle c_{m}\left|\rho_{C}\right| c_{m}\right\rangle$ are the diagonal elements of the clock in the preferred basis.

With these definitions in hand, we find that the clock generators take on the simple form of stochastic generators, namely the following.

Corollary 1: Let $\left(v_{C}^{0},\left\{\mathcal{M}_{C \rightarrow C T}^{\delta}\right\}_{\delta}\right)$ be a classical clock and suppose that the tick register has basis $\{|0\rangle,|1\rangle\}$. Then there exist $d \times d$-matrices $\mathcal{N}$ and $\mathcal{T}$ such that

$$
\begin{aligned}
\mathcal{M}_{C \rightarrow C T}^{\delta}\left(v_{C}\right)= & v_{C} \otimes|0\rangle\langle 0|+\delta\left(\mathcal{N} v_{C} \otimes|0\rangle\langle 0|\right. \\
& \left.+\mathcal{T} v_{C} \otimes|1\rangle\langle 1|\right)+O\left(\delta^{2}\right),
\end{aligned}
$$

with $\mathcal{T}$ being an entrywise positive matrix, and the sum $\mathcal{N}+\mathcal{T}$ being an infinitesimal generator (also known as a transition rate matrix). More precisely,

$$
\begin{aligned}
& \mathcal{N}_{m n} \begin{cases}\leq 0 & \text { for } m=n, \\
\geq 0 & \text { for } m \neq n,\end{cases} \\
& \mathcal{T}_{m n} \geq 0,
\end{aligned}
$$

for any $m, n$ and

$$
\sum_{m=1}^{d} \mathcal{N}_{m n}+\sum_{m=1}^{d} \mathcal{T}_{m n}=0
$$

for any $n$ [32].

See Appendix A 3 for a proof of this corollary. Analogous to the quantum case, we see that $\mathcal{T}$ is the classical version of the tick generator.

In the case of quantum clocks, we used the Lindbladian generators rather than the maps to describe the evolution of the clockwork as continuous and parametrized by $t$ (Sec. II B). We can do the same for classical clocks, by taking the limit $\delta \rightarrow 0$, as in Eq. (14). However, in the classical case, since the state is always diagonal with respect to a fixed orthonormal basis, we require only the dynamics of the vector of diagonal elements, which is seen to be

$$
\frac{d}{d t} v_{C}(t)=(\mathcal{N}+\mathcal{T}) v_{C}(t)
$$

As in the quantum case, in the continuous limit we replace the register by a probability density of a tick being recorded, Eq. (15), found to be

$$
P(t)=\left\|\mathcal{T} v_{C}(t)\right\|_{1}
$$

Furthermore, if one is focused on a single tick, as in Eq. (16), the reduced dynamics of the state of the clock for a single tick, $v_{C}^{(1)}$ is simply

$$
\frac{d}{d t} v_{C}^{(1)}(t)=\mathcal{N} v_{C}(t)
$$

\section{E. Accuracy of clocks}

As mentioned in the introduction, we use the regularity of the tick output of a clock as a measure for its accuracy. We now introduce definitions that allow us to express this quantity formally in terms of the clock maps.

A clock (Definition 1) after $N$ applications of the map $\mathcal{M}_{C \rightarrow C T}^{\delta}$ gives rise to a probability distribution $P_{T_{j}}\left(t_{N}\right)$ corresponding to the probability that $j-1$ ticks have occurred during the $N-1$ applications of the map, and the $j^{\text {th }}$ has occurred at the $N^{\text {th }}$ application of the map. In the limiting case of continuous clocks discussed in Sec. II B, the probability $P_{T_{j}}\left(t_{N}\right)$, converges to a probability density, such that $P_{T_{j}}(t) \delta t$ is the probability that $j-1$ ticks have occurred in the interval $[0, t)$ and the $j^{\text {th }}$ has occurred during the interval $[t, t+\delta t]$ for infinitesimal $\delta t>0$. Such probability densities are also known as delay functions. In particular, we call $P_{T_{j}}(t)$ the delay function associated with the $j^{\text {th }}$ tick. 
The expected time $\mu_{j}$ of the $j^{\text {th }}$ tick and its variance $\sigma_{j}^{2}$ are then given by

$$
\begin{aligned}
\mu_{j} & =\int_{0}^{\infty} d t P_{T_{j}}(t) t, \\
\sigma_{j}^{2} & =\int_{0}^{\infty} d t P_{T_{j}}(t)\left(t-\mu_{j}\right)^{2},
\end{aligned}
$$

for any $j \in \mathbb{N}_{>0}$. Based on these quantities, we can now define the clock accuracies $R_{j}$. Note that this is different from the clock accuracy $R$, which are defined below for the particular case of reset clocks.

Definition 4: The clock accuracies $\left(R_{j}\right)_{j \in \mathbb{N}_{>0}}$ of a clock $\left(\rho_{0},\left\{\mathcal{M}_{C \rightarrow C T}^{\delta}\right\}_{\delta}\right)$ is a sequence of real numbers, where the $j^{\text {th }}$ element $R_{j}$ is the accuracy of the $j^{\text {th }}$ tick,

$$
R_{j}=\frac{\mu_{j}^{2}}{\sigma_{j}^{2}} .
$$

We use this definition to define a partial ordering of clocks. For any two clocks $A$, and $B$, with clock accuracies $\left(R_{j}^{A}\right)_{j \in \mathbb{N}_{>0}}$ and $\left(R_{j}^{B}\right)_{j \in \mathbb{N}_{>0}}$, respectively, we say that $A$ is more accurate than $B$ if and only if every tick of $A$ is more accurate than the corresponding tick of $B$, i.e., if and only if $R_{j}^{A}>R_{j}^{B} \forall j \in \mathbb{N}_{>0}$. It is this definition that we refer to when we later prove that quantum clocks are more accurate than classical ones.

The characterization of clocks provided by Definition 4 has a number of nice properties. Firstly, it is scale invariant, meaning that the values $R_{j}$ are invariant under the mapping $t$ to $t / a$, for constants $a>0$. In other words, it is a measure of the accuracy of each tick, and is not affected by whether these ticks took place over a short or long time scale. Physically, this means that for every clock with accuracies $\left(R_{j}\right)_{j \in \mathbb{N}_{>0}}$, and mean tick times $\mu_{1}, \mu_{2}, \mu_{3}, \ldots$, there is another clock with the same accuracy, but with the ticks occurring on average at times $a \mu_{1}, a \mu_{2}, a \mu_{3}, \ldots$ The new clock is constructed from the old clock by mapping $t$ to $t / a$, which is equivalent to rescaling the generators $\left\{L_{j}\right\}_{j=1}^{m}$, $\left\{J_{j}\right\}_{j=1}^{m}$ and the Hamiltonian $H$, introduced in Lemma 1, by constant factors.

Furthermore, we can now appreciate the simplicity of reset clocks (Definition 2). Since every time such a clock produces a tick, it is reset to its initial state, the ticks represent a sequence of independent events, which are identically distributed. It thus follows that the delay function of the $j^{\text {th }}$ tick, $P_{T_{j}}(t)$, is the convolution of the delay function associated with the $1^{\text {st }}$ tick $P_{T_{1}}(t)$, with itself $j$ times. This in turn, gives rise to a simple relationship between the accuracies $\left(R_{j}\right)_{j \in \mathrm{N}_{>0}}$ of reset clocks (see Appendix B1 a, and Remark 6 for a detailed argument)

$$
R_{j}=j R_{1},
$$

and takes on a particularly satisfactory interpretation. Namely, the accuracy of the first tick $R_{1}$, is the number of ticks the clock generates (on average), before the next tick has a standard deviation equal to the mean time between ticks, $\mu_{1}$. As such, roughly speaking, the clock's useful lifetime is $\mu_{1} R_{1}$, beyond which one can no longer distinguish between subsequent ticks. To compare two reset clocks, it follows that one needs only to compare their $R_{1}$ values. Given the special significance of $R_{1}$, we sometimes simply refer to it as $R$.

A similar interpretation is also possible for the $R_{j}$ value of later ticks. For the purpose of illustration, suppose that the mean time between ticks, $\mu_{1}$ is one second. Then $R_{60}$ corresponds to the number of minutes (on average) that the clock can generate until the tick corresponding to the next minute has a standard deviation, which is equal to one minute. As such, while according to Eq. (31), $R_{60}$ is 60 times larger than $R_{1}$, this is not to say that "the 60th tick is more accurate than the first tick."

\section{FUNDAMENTAL LIMITATIONS FOR CLASSICAL AND QUANTUM CLOCKS}

In this section, we state our findings and explain their relevance and connection to related fields. There are two main theorems. The following one, which is about limitations on classical clocks, and Theorem 2, which shows how quantum clocks can outperform classical clocks.

Theorem 1: (Upper bound for classical clocks). For every d-dimensional classical clock, the clock accuracies $\left(R_{j}\right)_{j \in \mathbb{N}>0}$ satisfy

$$
R_{j} \leq j d
$$

for all $d \in \mathbb{N}_{>0}$. Furthermore, for every dimension $d \in$ $\mathbb{N}_{>0}$, there exists a reset clock whose accuracies $\left(R_{j}\right)_{j \in \mathbb{N}_{>0}}$ saturate the bound Eq. (32),

$$
R_{j}=j d .
$$

Proof. See Sec. II E for a proof of the above inequalities and Sec. C 1 for an explicit construction of an optimal $d$ dimensional reset clock, which saturates bound Eq. (32). This clock is further discussed in Fig. 3(a).

While the proof is a bit involved, there is an intuitive explanation to why reset clocks are optimal. If the clock is reset to its initial state after the first tick, then one can simply choose the initial state and dynamical map, which has the highest possible accuracy for the first tick. Intuitively, 
(a)

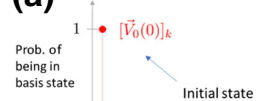

(b)

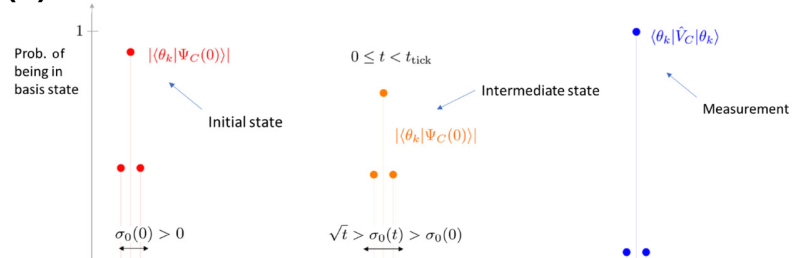

(c)

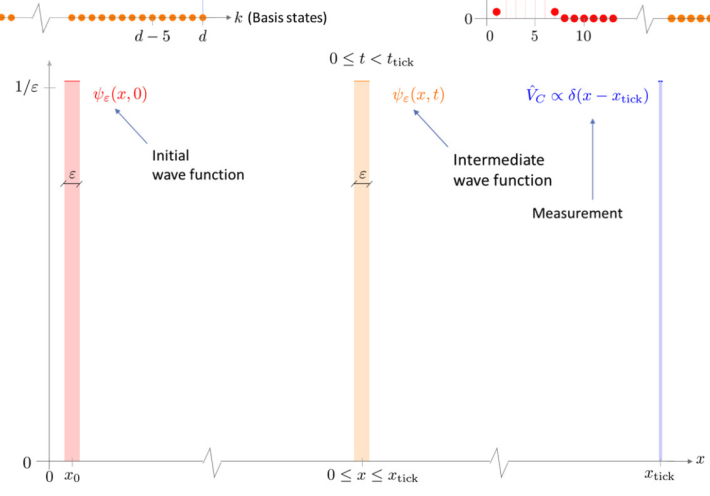

FIG. 3. Comparison of distributions of two clocks states before the first tick has occurred. (a) An optimal $d$-dimensional classical clock. (b) The quasi-ideal (quantum) clock [8]. (c) Pauli's idealized quantum clock. In a nutshell, the purpose of these figures is to highlight how classical clocks disperse more than quantum clocks. In contrast, a hypothetical perfect clock would not disperse at all. Intuitively, this is why quantum clocks can be more accurate than classical ones - but not perfect. $k$ labels orthogonal states; corresponding to real vectors in (a) and pure quantum states in (b). (a) The $d$-dimensional probability vector $V_{0}(t) \in \mathcal{P}_{d}$ associated with the clock having not ticked, starts off at $t=0$ with certainty at the first site, $V_{0}(0)=\mathbf{e}_{1}$ (red plot). Its mean $\mu_{0}(t)$ then moves with uniform velocity towards the right with a standard deviation $\sigma_{0}(t)$ increasing with $\sqrt{t}$ (orange plot). The tick generator $\mathcal{T}$, is chosen so that the clock can only "tick" from the last site, $\mathbf{e}_{d}$ and the clock is reset (blue plot). This clock, whose full details are reserved for Appendix C, will "tick" once it has reached the site $n=d$, at which point it will have dispersed considerably, and as such, its accuracy is limited to $R_{1}=d$. Furthermore, since it is a reset clock, later ticks are optimally accurate, $R_{j}=j d$. (b) The quasi-ideal clock starts in a distribution in the time basis, which is highly peaked around $\left|\theta_{0}\right\rangle$, resulting in a small standard deviation $\sigma_{0}$ in the time basis (red plot). The amplitudes of its distribution shift and move in time towards a large concentration around $\left|\theta_{d-1}\right\rangle$, where a "tick" is measured with high probability and the clock is reset to its initial state (blue plot). During the time intervals between ticks this clock will disperse, but less than the classical clock in (a) due to quantum interference. This results in a smaller standard deviation $\sigma_{0}(t)$ than in (a) (orange plot). Furthermore, it will also be disturbed by time measurements, causing further unwanted dispersion. However, there is a trade-off - the smaller the standard deviation of the initial state, the more precise initial time measurements will be, but the larger the dispersion due to dynamics and measurements will be too, making later time measurements less reliable. Even so, due to quantum constructive and destructive interference, quantum mechanics allows for a $d$-dimensional state to disperse less as it travels to the region where a tick has the highest probability of occurring, than an optimal $d$-dimensional classical clock, such as in (a). As such, this quantum clock can surpass the classical bound; see Theorem 2. (c) The idealized clock of Pauli starts with an arbitrarily highly peaked wave function at position $x=0$ (red plot). It then moves according to $\psi(t, x)=\psi(0, x-t)$; towards $x=x_{\text {tick }}$ (orange plot). At all times, its standard deviation is a constant $\epsilon>0$, which can be chosen to be arbitrarily small. It is not disturbed at all by time measurements, and "ticks" exactly at time $t_{\text {tick }}=x_{\text {tick }}$ (blue plot), resulting in perfect accuracy $R_{1}=\infty$. Furthermore, one can add additional Dirac delta distributions to the potential centred around $2 x_{\text {tick }}, 3 x_{\text {tick }}, \ldots$ without effecting the standard deviation of the idealized clock. This results in perfect accuracy for all later ticks; $R_{2}=R_{3}=\ldots=\infty$.

the only way a nonreset clock could have a superior accuracy for later ticks than this one, would be for one to adjust the mean time of the following tick in the sequence to be longer or shorted than the previous one to make up for any lost or gained time due to the previous tick being "early" or "late." However, determining whether the clock ticked too early or late would require an additional clock, which is not available within the model.
The optimal reset clocks, which saturate the bound in Eq. (32), provide insight into our results. For these classical clock examples, the clock starts at one end of a length- $d$ nearest-neighbor chain, with the tick generator's support region located at the other end. The clock dynamics produce a classical continuous biased random walk along this chain, see Fig. 3(a) for details. The error in telling the time is a consequence of the state dispersing as it travels along 
the chain. Indeed, for these clocks, the standard result from random-walk theory, which predicts that the standard deviation in a state is proportional to the square root of the distance traveled, approximately holds. This dispersive behavior achieves $R_{1}=d$ in the optimal case.

Before making a comparison with the quantum clocks described in this paper, it is illustrative to compare this result with a recent clock in the literature [9]. Here a quantum clock is powered by two thermal baths, at different temperatures. This temperature difference drives a random walk of an atomic particle up a $d$-dimensional ladder, which spontaneously decays back to the initial state when reaching the top of the ladder, emitting a "tick" in the decay process. As such, it is a reset clock whose accuracy $R_{1}$ depends on the entropy generated by the clock. Interestingly, in the limit of weak coupling and vanishing frequency of ticks, it is found [9] that the clock dynamics becomes classical, represented by a biased random walk up the ladder. The accuracy of the clock is then

$$
R \approx d\left(\frac{p_{\uparrow}-p_{\downarrow}}{p_{\uparrow}+p_{\downarrow}}\right) \approx d\left(\frac{e^{\Delta S / d}-1}{e^{\Delta S / d}+1}\right),
$$

where $p_{\uparrow}, p_{\downarrow}$ are the probabilities of moving up and down the ladder, induced by the thermal baths, and $\Delta S$ is the entropy generated by the clock for each instance that it ticks. As such, as far as the criterion of dimensionality is concerned, this classical thermodynamic clock is always less accurate than the optimal classical clock in Theorem 1 by a constant factor, and approaches only optimal accuracy in the limit of infinite entropy generation $\Delta S \rightarrow \infty$.

On the other hand, we can also compare the classical clock in Fig. 3(a) with the behavior of the idealized clock of Pauli, introduced in Sec. II. Here the clock Hamiltonian is the generator of translations, and it is not disturbed by continuous measurements, thus leading to no dispersion, and a clock accuracy of $R_{1}=\infty$, see Fig. 3(c). Of course, as previously discussed, this high accuracy is unfortunately an artefact of requiring both infinite energy and dimension.

The important question is whether one can do better than the classical clock, and achieve higher accuracy with a quantum clock. For this task, we consider the quasi-ideal clock introduced in Sec. II, which is formed by taking a complex Gaussian amplitude superposition of the SWP clocks, namely

$$
\left|\Psi_{\text {quasi-ideal }}\right\rangle:=\sum_{k \in \mathcal{S}_{d}\left(k_{0}\right)} A e^{-\pi / \sigma_{0}^{2}\left(k-k_{0}\right)^{2}} e^{i 2 \pi n_{0}\left(k-k_{0}\right) / d}\left|\theta_{k}\right\rangle,
$$

where $\mathcal{S}_{d}\left(k_{0}\right)$ is a set of $d$ consecutive integers centered about $k_{0} \in \mathbb{R}, n_{0} \in(0, d)$ determines the mean energy of the clock state and $\sigma_{0}$ its width in the $\left|\theta_{k}\right\rangle$ basis. Its clock Hamiltonian $\hat{H}_{C}$ is the first $d$ levels of a quantum harmonic oscillator with level spacing $\omega ; \hat{H}_{C}=\sum_{n=0}^{d-1} n \omega\left|E_{n}\right\rangle\left\langle E_{n}\right|$.
The dynamics of the clock is generated via the (possibly non-Hermitian) operator $\hat{H}=\hat{H}_{C}+\hat{V}_{C}$, where $\hat{V}_{C}=$ $\sum_{i=0}^{d-1} V_{i}\left|\theta_{i}\right\rangle\left\langle\theta_{i}\right|$ with, $\left\{\left|\theta_{k}\right\rangle\right\}$ the complementary basis to $\left\{\left|E_{k}\right\rangle\right\}$, formed by taking the discrete Fourier transform. As such, $\hat{H}_{C}$ and $\hat{V}_{C}$ are diagonal in complementary bases to each other. This setup was introduced in Ref. [8] with the aim of studying unitary control of other quantum systems. For this objective, the clock underwent unitary dynamics without being measured. Here we use its construct to see how well quantum clocks can measure time. Indeed, the potential $\hat{V}_{C}$ can be chosen to correspond to continuous measurements rather than unitary evolution by making it anti-Hermitian instead of Hermitian. It then follows that one can use the quasi-ideal clock setup to perform continuous measurements on a reset clock as described in Sec. II C. In particular, the dynamics under $\hat{H}=\hat{H}_{C}+\hat{V}_{C}$ takes the same form as Eq. (22) on making the basis identification $\left\{\left|\theta_{k}\right\rangle=\left|t_{k}\right\rangle\right\}_{k}$-which we now make.

If one chooses the width of the Gaussian in Eq. (35) to be $\sigma_{0}=\sqrt{d}$, then the width in the complementary basis $\left\{\left|E_{k}\right\rangle\right\}$ is also $\sqrt{d}$. In such cases, an accuracy $R_{1}$ proportional to $d$ can be achieved. However, if we choose a width that is narrower but not too narrow, namely $\sigma_{0}=d^{\eta / 2}$ for small $\eta>0$, then the quasi-ideal clock is able to mimic approximately the dynamical behavior of the idealized clock, while maintaining finite energy and dimension [8], see Fig. 3(b). The following theorem formalizes this. In addition to $\sigma_{0}=d^{\eta / 2}$, other parameters such as the particular potential $\hat{V}_{C}$ and coefficients $n_{0}, k_{0}$ used in the theorem are specified in the proof.

Theorem 2: (Achievable accuracy for quantum clocks.) Consider the quantum clock construction in Sec. II C and use a quasi-ideal clock. For all fixed constants $0<\eta \leq$ 1 , and appropriately chosen parameters, the quasi-ideal clock's accuracy satisfies

$$
R_{1} \geq d^{2-\eta}+o\left(d^{2-\eta}\right)
$$

in the large d limit, where we use little-o notation. Furthermore, since it is a reset clock; the accuracies $\left(R_{j}\right)_{j \in \mathbb{N}_{>0}}$ of later ticks satisfy

$$
R_{j}=j R_{1}
$$

for all $j, d \in \mathbb{N}_{>0}$.

Proof. See Sec. F for a proof of a slightly more general version of the theorem. The main difficulty of the proof is to come up with a potential $\hat{V}_{C}$, which satisfies all the necessary properties - if its derivatives are too large, the clock dynamics are too disturbed by the continuous measurements, yet if they are not large enough, the measurements will not capture enough time information from the clock. 


\section{DISCUSSION OF THE QUANTUM BOUND: RELATIONSHIP TO RELATED FIELDS AND OPEN PROBLEMS}

In this section, we discuss the relationship of the quantum bound to other concepts and fields, which have been associated with time and clocks in quantum mechanics in the past: time-energy uncertainty relations, quantum metrology, quantum speed limits.

\section{A. Time-energy uncertainty relation}

The time-energy uncertainty relation,

$$
\Delta t \Delta E \geq \frac{1}{2},
$$

has been a controversial concept ever since its conception during the early days of quantum theory, with Bohr, Heisenberg, Pauli, and Schrödinger giving it different interpretations and meanings. It still remains a controversial issue to this day, and multiple interpretations have been found $[33,34]$. Often, at the heart of the controversy, is that in quantum mechanics, as explained in the introduction, Sec. I, time is usually associated with a parameter, rather than an operator.

Since in the present context, we do have operators for time, a lot of this controversy can be circumvented. Indeed, Peres introduced a time operator, $\hat{t}_{c}:=$ $\sum_{k=0}^{d-1}\left(T_{0} / d\right) k\left|\theta_{k}\right\rangle\left\langle\theta_{k}\right|$, where $T_{0}=2 \pi / \omega$ is the period of the clock Hamiltonian $\hat{H}_{C}$ [19]. In Ref. [8] it was shown that the standard deviation of the initial clock state Eq. (35) saturates a time-energy uncertainty relation; $\Delta E \Delta t=1 / 2$ [35], where the standard deviations $\Delta t, \Delta E$ are calculated using the operators $\hat{t}_{c}$ and $\hat{H}_{C}$, respectively. One may be inclined to believe that one can increase the accuracy of the clock, by decreasing $\Delta t$ as a consequence of a larger $\Delta E$. While indeed decreasing $\sigma_{0}$ does have this effect, it would be naive to believe this paints the full picture.

To study this effect as the clock moves around the clockface, let $\Delta E(t)$ be the standard deviation of the clock coefficients dephased in the energy basis, $\left\{\left\langle E_{n}\left|\rho_{C}(t)\right| E_{n}\right\rangle\right\}_{n=0}^{d-1}$. We define the standard deviation in the time basis similarly, but here one has to be careful since the time basis $\left\{\left|\theta_{k}\right\rangle\right\}$ has circular boundary conditions, meaning $\left|\theta_{k}\right\rangle=$ $\left|\theta_{k+1}(\bmod d)\right\rangle$. Consequently, the state will "jump" from the state $\left|\theta_{d-1}\right\rangle$ to $\left|\theta_{0}\right\rangle$ as it completes one period of its motion. We are not interested in the jumps due to the boundary effects, and therefore denote $\Delta C(t)$ the standard deviation of the coefficients $\left\langle\theta_{k}\left|\rho_{C}(t)\right| \theta_{k}\right\rangle$ with the circular boundary conditions replaced with open boundaries [36]. This way, we mimic the hands of a real clock, which visually do not suddenly "jump" at 12 O'Clock. The quantity $\Delta C(t)$ is plotted for the quasi-ideal clock and SWP clocks in Fig. 4(a).

Firstly, if $\sigma_{0}$ is too small, the clock will disperse too much due to its dynamics and time measurements. (a) $\Delta c$

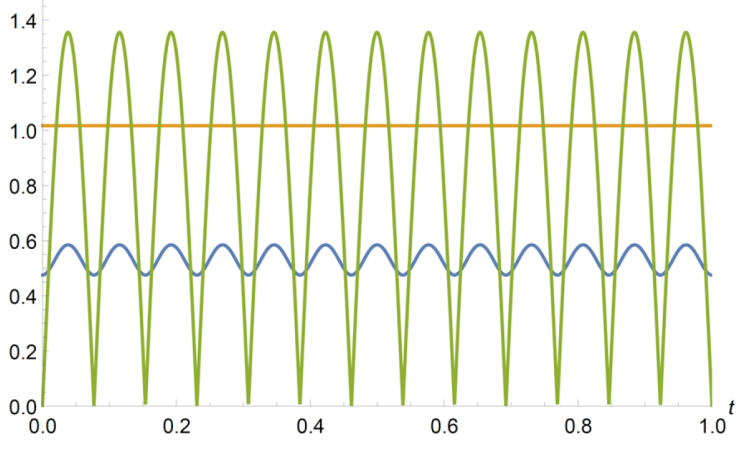

(b)

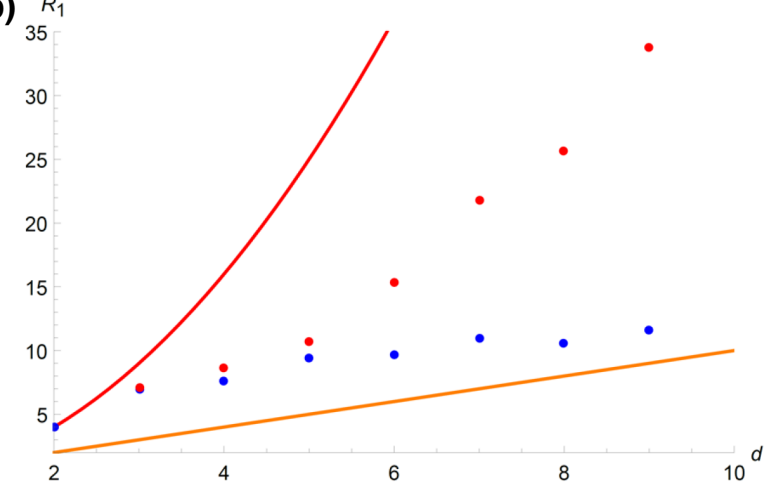

FIG. 4. Comparison of the quasi-ideal and SWP clocks. (a) Standard deviations in the time basis as a function of time. (b) $R_{1}$ as a function of clock dimension $d$. (a) Standard deviation of clock states in the time basis $\Delta C(t)$ for different clocks as a function of time $t$, when time evolved according to their clock Hamiltonian $\hat{H}_{C}=\sum_{n=0}^{d-1} \omega n\left|E_{n}\right\rangle\left\langle E_{n}\right|$. Time runs from zero to one clock period $T_{0}=1$ with clock dimension $d=13$. Initial states are as follows: quasi-ideal clock states for $\sigma_{0}=\sqrt{\bar{d}} \approx 3.61$ (orange), $\sigma_{0}=1.8$ (blue), and a SWP state (green). (b) Numerical optimization of $R_{1}$ for the quasi-ideal quantum clock (red data points) and SWP quantum clock (blue data points) for a set of potentials. Both quasi-ideal and SWP achieve $R_{1}=4$ for $d=2$; however, for large dimensions, the quasi-ideal clock achieves higher accuracy. Red and orange solid lines $\left(R_{1}=d^{2}\right.$ and $R_{1}=d$, respectively) are guides to the eye, which represent the lower asymptotic bound for the quasi-ideal clock and the upper bounds for the optimal classical clock respectively. See Theorems 2 and 1.

Secondly, another way to decrease $\Delta t$ and increase $\Delta E$, is via reducing $T_{0}$, yet this has no effect on the accuracy of the clock. This latter observation is also related to how our measure of accuracy $R_{1}$, is invariant under a rescaling of time, as discussed in Sec. I. In other words, for the lower bound in Eq. (36) to exceed the classically permitted value of $d$, the clock uncertainty in the time basis $\Delta t$, must be smaller than the uncertainty in the energy basis $\Delta E$ during the time in which the clock is running. In Fig. 4(a), the orange plot has $\Delta C(t) \approx \Delta E(t) \approx \sqrt{1 / 2}$. This is suboptimal, since the POVMs used to measure ticks are diagonal in the time basis $\left\{\left|\theta_{k}\right\rangle\right\}$. Furthermore, the blue curve has a 
smaller $\Delta C(t)$, and according to Theorem 2 can achieve $R_{1}>d$. However, if we continue to choose initial clock states with smaller $\Delta C(0)$, we run into a problem, namely in the limit $\sigma_{0} \rightarrow 0^{+}$, we recover the SWP clock state (green), which has a very large $\Delta C(t)$ at later times.

Another interesting aspect regarding time and energy, is what is the internal energy of the clock. We can easily answer this question for the quasi-ideal clock. Note that for $n_{0}=(d-1) / 2$, the mean energy of the quasi-ideal clock is $\omega d / 2+o(d)$; thus Theorem 2 implies that high accuracy can be achieved with a modest linear increase in internal energy.

\section{B. Quantum metrology}

Metrology is the science of measuring unknown parameters. Here, like in other areas of quantum science, it has been shown that allowing for quantum effects can vastly improve the precision of measurements in comparison with the optimal classical protocols. Quantum metrology is now a mature discipline with a vast literature [37]; and as such, it is appropriate to compare our results about clocks with them.

The basic setup in metrology is as follows. When attempting to measure an unknown parameter in a physical system, we prepare a probe state, let it evolve, and finally measure the evolved system. The evolution stage is according to the unitary operator $U_{x}:=e^{-i x \hat{H}}$, where $\hat{H}$ is a known Hamiltonian.

The model of a clock described in this paper differs substantially from the metrology setup above. The easiest way to convey the main difference is via a simple example: for the purpose of illustration, imagine you were told to perform a particular task after $5 \mathrm{~s}$. If you had a clock you could wait for 5 ticks to pass according to it, and perform your designated task. However, in the case of the metrology setup one can estimate only the time when one happened to measure the probe. As discussed in Sec. I, this marks an important division between timekeeping devices, and those based on the standard metrology setups described here, fall into the category of stopwatches and not clocks, according to our definitions. One could of course use a very accurate stopwatch in combination with a not so accurate clock, by measuring the stopwatch when the clock ticks, and resetting the stopwatch immediately after measuring it. While one would know to high accuracy when in time these ticks occurred, their distribution in time would still be the same as if we did not have access to the stopwatch. As such, we can observe that even this combination cannot be substituted for a more accurate clock.

\section{Quantum speed limits}

The quantum speed limit, $\tau_{\mathrm{QSL}}$ is the minimum time $t_{\mathrm{QSL}}$ required for a state $|\psi(0)\rangle$ to become orthogonal to itself, $\left\langle\psi(0) \mid \psi\left(t_{\mathrm{QSL}}\right)\right\rangle=0$ under unitary time evolution, $|\psi(t)\rangle=e^{-i t \hat{H}}$. The celebrated Margolus-Levitin and Mandelstam-Tamm bounds, impose a tight lower limit in terms of the mean and standard deviation of $\hat{H}$ with respect to the initial state $|\psi(0)\rangle$ [38]. It has found many applications in the field of thermodynamics, metrology, and the study of the rate at which information can be transmitted from a quantum system to an external observer $[39,40]$.

One may also be inclined to think that the fundamental limitations on the accuracy of clocks is related to how quickly the initial clock state becomes perfectly distinguishable to itself, when measured by an external observer. Indeed, the quicker states become distinguishable, the faster one can extract timing information from the clock state. Unfortunately, a simple example will reveal how the situation of a clock is too subtle to be captured by such simple arguments. The quantum speed limit $t_{\mathrm{QSL}}$ of the SWP clock is precisely $T_{0} / d=2 \pi /(\omega d)$, since the states satisfy $e^{-i \hat{H}_{C} T_{0} / d}\left|\theta_{k}\right\rangle=\left|\theta_{k+1}\right\rangle$. On the other hand, the quasi-ideal clock has a quantum speed limit of much larger than $T_{0}$. Yet, as described in Fig. 4(b), numerics predict that while the SWP clock has a higher accuracy than the optimal classical clock, it is less accurate than the quasi-ideal clock. The explanation of this is that, contrary to the quasi-ideal clock, the SWP clock - at the expense of a shorter quantum speed limit time - becomes highly spread out in the time basis for times in between becoming orthogonal to itself, thus incurring large $\Delta C(t)$ during these times [see green plot in Fig. 4(a)]. In summary, the quantum speed limit only tells us about how long it takes for a clock state to become distinguishable to itself, but fails to quantify the behavior in between. Since a clock has to produce a continuous stream of "tick," "no-tick" information, the nature of dynamics of the clock at all times is of high importance.

\section{CONCLUSION AND OUTLOOK}

The workings of a clock requires a subtle interplay between two themes, measurement and dynamics - measure the time marked by the clock too strongly, and its dynamics will be very disturbed, adversely affecting later measurements of time. Yet measure too weakly, and you will not gain much information about time at all. Furthermore, the optimal state for minimizing measurement disturbance, may possess a suboptimal dynamical evolution for distinguishing between different times - this poses another trade-off. Finding the optimal clock under measurements and dynamics is a fundamental and challenging problem.

Here we motivate and use a general framework where any clock is regarded as a $d$-dimensional system that autonomously emits information about time (the ticks) to the outside [6], and we show a quantum-over-classical advantage for the accuracy of this time information: to achieve the same accuracy as a classical clock of size $d$ a quantum clock requires only, roughly, dimension $\sqrt{d}$. 
A quantum-over-classical advantage characterized quantitatively by a square root is known for other tasks, in particular, database search (where Grover's algorithm provides an advantage over any classical algorithm) or quantum metrology (where joint measurements provide an advantage over individual measurements). We stress, however, that these results are all of a different kind (see Sec. IV). In the case of database search, the relevant quantity is the number of blackbox accesses to the database. In the case of metrology, the timekeeping devices that one typically encounters, can predict only the time when they happened to be measured; thus, they are more akin to stopwatches than clocks, which autonomously emit a periodic time reference.

Traditionally, quantum metrology and concepts such as the time-energy uncertainty relation, and quantum speed limits; have been associated with characterizing how precisely different physical processes involving time can be carried out. Here we see that while these concepts have a role to play, a more discerning feature between the accuracy of classical and quantum clocks is how continuoustime quantum walks, under the right circumstances, allow for a smaller spread in the mean distribution, compared to classical standard walks, which are limited to a standard deviation, which is proportional to the square root of the mean distance traveled.

Finally, we turn to discussing potential practical applications of our results. Given the description of a quantum clock with its corresponding Hamiltonian, one may understand the error in the clock's signal as arising from two sources. Firstly, the fundamental limitation of the clock due to its dimension, that we expose here, and secondly, the error due to its Hamiltonian not being perfectly stable. It is the second type that is the dominant challenge for current atomic clocks that work by frequency stabilization. In order for our result to become practically relevant, the control over energy levels must increase to the point that the fundamental limitation exposed here becomes dominant.

At the point where this is attained, the accuracy will be higher than that of current clocks, whose fractional error (the inverse of $R$ ) is of the order of $10^{-19}$ [41]. Thus, the effective dimension [42] of a quantum clock that attains this level of accuracy must be at least of the order of $10^{10}$.

These two conditions: stable Hamiltonians and highdimensional coherent control, will allow us to build even more accurate clocks by making full use of quantum properties, as demonstrated in this paper. A dimension of $10^{10}$ is attainable by 33 qubits, which is not entirely outside the realm of possibility, given the current state of affair in quantum-computing technology [43].

Another potential reason for pursuing a quantum clock is to explore the role quantum mechanics plays in gravity. Some theoretical models for quantum gravity predict that the general relativistic effect of time dilation will be slightly altered by quantum theory. For example, one semiclassical approach has predicted that while classical stopwatches are governed by standard time dilation when in the presence of a gravitational field, quantum stopwatches convey a, small yet important, modified time due to quantum fluctuations $[44,45]$. Similar effects have been reported in interferometry too [46]. Therefore, while two clocks of the same precision (one classical, the other quantum) may be just as precise for telling the time, the quantum one has added value as a probe of quantum gravity. Recently, other foundational questions regarding clocks have been made, for example, how they can be derived from axiomatic principles [22], and how the precision of a quantum clock is related to a violation of Leggett-Gargtype inequalities, which provide further insight into their non classical nature [47].

\section{ACKNOWLEDGMENTS}

We thank Carlton Caves, Nicolas Gisin, Dominik Janzing, Christian Klumpp, Yeong-Cherng Liang and Yuxiang Yang for stimulating discussions. We all acknowledge the Swiss National Science Foundation (SNSF) via Grant No. 200020_165843 and via the NCCR QSIT, Foundations Questions Institute via Grant No. FQXi-RFP-1610. M.W. acknowledges funding from his personal FQXi Grant Finite dimensional Quantum Observers (No. FQXiRFP-1623) for the programme Physics of the Observer. R.S. acknowledges funding from the SNSF via Grant No. 200021_169002, which funded him while at the University of Geneva.

\section{Author Contributions}

All authors contributed to the ideas and results within this work. M.W. proved the lower bound for quantum clocks (Theorem 2). R.S. proved the upper bound on classical clocks (Theorem 1). S.S. provided numerics on quantum clocks (Fig. 4). M.W. and R.R. wrote the main text.

\section{APPENDIX A: MODELING OF CLOCKS}

\section{Proof of lemma 1}

Proof. Consider an operator-sum representation of the map $\mathcal{M}_{C \rightarrow C T}^{\delta}$, i.e.,

$$
\begin{aligned}
\mathcal{M}_{C \rightarrow C T}^{\delta}\left(\rho_{C}\right)= & \sum_{j=0}^{m} M_{j} \rho_{C} M_{j}^{\dagger} \otimes|0\rangle\left\langle\left. 0\right|_{T}\right. \\
& +\sum_{j=1}^{n} N_{j} \rho_{C} N_{j}^{\dagger} \otimes|1\rangle\left\langle\left. 1\right|_{T},\right.
\end{aligned}
$$

where $\left\{M_{j}\right\}_{j=0}^{m}$ and $\left\{N_{j}\right\}_{j=1}^{n}$ are families of operators on $C$. We assume without loss of generality that the labeling of 
the operators $M_{j}$ is such that $\left\|M_{0}\right\| \geq\left\|M_{j}\right\|$ for any $j \neq 0$. Define furthermore Hermitian operators $H$ and $K$ such that

$$
K-i H=\frac{1}{\delta}\left(M_{0}-\mathbb{1}\right)
$$

and

$$
\begin{aligned}
& L_{j}=\frac{1}{\sqrt{\delta}} M_{j}, \\
& J_{j}=\frac{1}{\sqrt{\delta}} N_{j} .
\end{aligned}
$$

Furthermore, we can assume without loss of generality that $n=m$. The operators $M_{j}$ and $N_{j}$ can be chosen such that $K, H, L_{j}$, and $J_{j}$ are independent of $\delta$ without loss of generality, which one can prove as follows. From Eq. (A1) it follows that

$$
\mathcal{M}_{C \rightarrow C}^{\delta}\left(\rho_{C}\right)=\sum_{j=0}^{m} M_{j} \rho_{C} M_{j}^{\dagger}+\sum_{j=1}^{n} N_{j} \rho_{C} N_{j}^{\dagger} .
$$

Given the defining properties stated in Sec. II A, Lindblad proved [25] that the map $\mathcal{M}_{C \rightarrow C}^{\delta}\left(\rho_{C}\right)$ can be written as a Lindbladian with semigroup parameter $\delta$ [48]. By Taylor expanding the generic expression for $\mathcal{M}_{C \rightarrow C}^{\delta}\left(\rho_{C}\right)$ when expressed in Lindblad form to first order in $\delta$, and equating zeroth- and first-order terms with the right-hand side of Eq. (A4), the dependency on $\delta$ in Eqs. (A2) and (A3) follows.

Equation (A1) then reads

$$
\begin{aligned}
& \mathcal{M}_{C \rightarrow C T}^{\delta}\left(\rho_{C}\right) \\
& =\left(\mathbb{1}_{C}+\delta K-\delta i H\right) \rho_{C}\left(\mathbb{1}_{C}+\delta K+\delta i H\right) \otimes|0\rangle\left\langle\left. 0\right|_{T}\right. \\
& \quad+\sum_{j=1}^{m} \delta L_{j} \rho_{C} L_{j}^{\dagger} \otimes|0\rangle\left\langle\left. 0\right|_{T}+\sum_{j=1}^{m} \delta J_{j} \rho_{C} J_{j}^{\dagger} \otimes \mid 1\right\rangle\left\langle\left. 1\right|_{T} .\right.
\end{aligned}
$$

We then have to first order in $\delta$

$$
\begin{aligned}
\mathcal{M}_{C \rightarrow C T}^{\delta}\left(\rho_{C}\right) & \\
= & \rho_{C} \otimes|0\rangle\left\langle\left. 0\right|_{T}+\delta(\{K, \rho\}-i[H, \rho]) \otimes \mid 0\right\rangle\langle 0| \\
& +\delta \sum_{j=1}^{m}\left(L_{j} \rho_{C} L_{j}^{\dagger} \otimes|0\rangle\left\langle\left. 0\right|_{T}+J_{j} \rho_{C} J_{j}^{\dagger} \otimes \mid 1\right\rangle\left\langle\left. 1\right|_{T}\right)\right. \\
& +O\left(\delta^{2}\right) .
\end{aligned}
$$

The requirement that $\mathcal{M}_{C \rightarrow C T}^{\delta}$ be trace-preserving furthermore implies that

$$
\begin{gathered}
\left(\mathbb{1}_{C}+\delta K+\delta i H\right)\left(\mathbb{1}_{C}+\delta K-\delta i H\right) \\
+\delta \sum_{j=1}^{m}\left(L_{j}^{\dagger} L_{j}+J_{j}^{\dagger} J_{j}\right)=\mathbb{1}_{C} .
\end{gathered}
$$

Hence, again to first order in $\delta$, we have

$$
2 \delta K=-\delta \sum_{j=1}^{m}\left(L_{j}^{\dagger} L_{j}-J_{j}^{\dagger} J_{j}\right)+O\left(\delta^{2}\right) .
$$

Inserting this into the above yields the claim.

Finally, to prove the converse part of the lemma, we first note that for any Hermitian operator $H$ and families of orthogonal operators $\left\{L_{j}\right\}_{j=1}^{m},\left\{J_{j}\right\}_{j=1}^{m}$ from $\mathcal{H}_{C}$ to $\mathcal{H}_{C}$, the reduced map $\mathcal{M}_{C \rightarrow C}^{\delta}(\cdot)$ in Eq. (12) takes on the following form:

$$
\mathcal{M}_{C \rightarrow C}^{\delta}(\cdot)=\operatorname{tr}_{T} \circ \mathcal{M}_{C \rightarrow C T}^{\delta}(\cdot)=e^{\delta \mathcal{L}}(\cdot)+O\left(\delta^{2}\right),
$$

with the Lindbladian

$$
\begin{aligned}
\mathcal{L}(\cdot)= & -\left(i[H,(\cdot)]+\sum_{j=1}^{m} \frac{1}{2}\left\{L_{j}^{\dagger} L_{j}+J_{j}^{\dagger} J_{j},(\cdot)\right\}-L_{j}(\cdot) L_{j}^{\dagger}\right) \\
& +\sum_{j=1}^{m} J_{j}(\cdot) J_{j}^{\dagger} .
\end{aligned}
$$

We thus have

$$
\begin{aligned}
& \lim _{\Delta \rightarrow 0} \lim _{\delta \rightarrow 0}\left(\operatorname{tr}_{T} \circ \mathcal{M}_{C \rightarrow C T}^{\delta}\right)^{\left\lfloor\frac{\Delta}{\delta}\right\rfloor} \\
& \quad=\lim _{\Delta \rightarrow 0} \lim _{\delta \rightarrow 0}\left[e^{\delta \mathcal{L}}+O\left(\delta^{2}\right)\right]^{\left\lfloor\frac{\Delta}{\delta}\right\rfloor} \\
& \quad=\lim _{\Delta \rightarrow 0} \lim _{\delta \rightarrow 0}\left[e^{\delta\left\lfloor\frac{\Delta}{\delta}\right\rfloor \mathcal{L}}+O\left(\lfloor\Delta / \delta\rfloor \delta^{2}\right)\right] \\
& =\lim _{\Delta \rightarrow 0} e^{\Delta \mathcal{L}}=\mathcal{I}_{C},
\end{aligned}
$$

proving that the map $\mathcal{M}_{C \rightarrow C T}^{\delta}(\cdot)$ satisfies Eq. (9) in Definition 1 and thus is a clock.

\section{Lindbladian semigroup for clock and register qubit}

While Eq. (12) of the main text does not define a dynamical semigroup $C T \rightarrow C T$, one can do so quite simply via

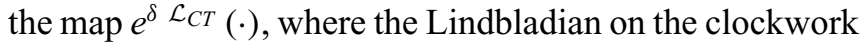
and register is

$$
\begin{aligned}
\mathcal{L}_{C T}(\cdot)= & -i[\tilde{H},(\cdot)]+\sum_{j=1}^{m} \sum_{a \in 0,1} \tilde{L}_{j a}(\cdot) \tilde{L}_{j a}^{\dagger}-\frac{1}{2}\left\{\tilde{L}_{j a}^{\dagger} \tilde{L}_{j a},(\cdot)\right\} \\
& +\sum_{j=1}^{m} \tilde{J}_{j}(\cdot) \tilde{J}_{j}^{\dagger}-\frac{1}{2}\left\{\tilde{J}_{j}^{\dagger} \tilde{J}_{j},(\cdot)\right\},
\end{aligned}
$$

where the extended operators are $\tilde{H}=H \otimes \mathbb{1}_{T}, \tilde{L}_{j 0}=$ $L_{j} \otimes|0\rangle\left\langle\left. 0\right|_{T}, \tilde{L}_{j 1}=L_{j} \otimes \mid 1\right\rangle\left\langle\left. 1\right|_{T}\right.$, and $\left.\tilde{J}_{j}=J_{j} \otimes \mid 1\right\rangle\left\langle\left. 0\right|_{T}\right.$. The clock map is then defined via

$$
\mathcal{M}_{C \rightarrow C T}^{\delta}\left(\rho_{C}\right)=e^{\delta \mathcal{L}_{C T}}\left(\rho_{C} \otimes|0\rangle\left\langle\left. 0\right|_{T}\right) .\right.
$$




\section{Proof of corollary 1}

We prove the corollary using density-matrix notation.

Corollary 1: Let $\left(\rho_{C}^{0},\left\{\mathcal{M}_{C \rightarrow C T}\right\}\right)$ be a classical clock with basis $\left\{|c\rangle_{j}\right\}_{j=1}^{d}$ and suppose that the tick register has basis $\{|0\rangle,|1\rangle\}$. Then there exist $d \times d$-matrices $\mathcal{N}$ and $\mathcal{T}$ such that

$$
\begin{aligned}
\mathcal{M}_{C \rightarrow C T}^{\delta}\left(\rho_{C}\right)= & \rho_{C} \otimes|0\rangle\left\langle 0\left|+\delta \sum_{m, n}\left\langle c_{n}\left|\rho_{C}\right| c_{n}\right\rangle\right| c_{m}\right\rangle\left\langle\left. c_{m}\right|_{C}\right. \\
& \otimes\left(\mathcal{N}_{m n}|0\rangle\left\langle\left. 0\right|_{T}+\mathcal{T}_{m n} \mid 1\right\rangle\left\langle\left. 1\right|_{T}\right)+O\left(\delta^{2}\right) .\right.
\end{aligned}
$$

with

$$
\begin{aligned}
& \mathcal{N}_{m n} \begin{cases}\leq 0 & \text { for } m=n, \\
\geq 0 & \text { for } m \neq n,\end{cases} \\
& \mathcal{T}_{m n} \geq 0,
\end{aligned}
$$

for any $m, n$, and

$$
\sum_{m=1}^{d} \mathcal{N}_{m n}+\sum_{m=1}^{d} \mathcal{T}_{m n}=0
$$

for any $m$.

Proof. Let $L_{j}$ and $J_{j}$ be the operators defined by Lemma 1 and set

$$
\begin{aligned}
\mathcal{N}_{m n}= & -\delta_{m, n}\left\langle c_{m}\left|\sum_{j}\left(L_{j}^{\dagger} L_{j}+J_{j}^{\dagger} J_{j}\right)\right| c_{m}\right\rangle \\
& +\sum_{j}\left|\left\langle c_{m}\left|L_{j}\right| c_{n}\right\rangle\right|^{2}, \\
\mathcal{T}_{m n}= & \sum_{j}\left|\left\langle c_{m}\left|J_{j}\right| c_{n}\right\rangle\right|^{2} .
\end{aligned}
$$

It is then straightforward to verify the claimed expression for the map. Furthermore, for any $n \in\{1, \ldots, d\}$,

$$
\begin{aligned}
\sum_{m=1}^{d}\left(\mathcal{N}_{m n}+\mathcal{T}_{m n}\right)= & -\left\langle c_{n}\left|\sum_{j}\left(L_{j}^{\dagger} L_{j}+J_{j}^{\dagger} J_{j}\right)\right| c_{n}\right\rangle \\
& +\sum_{j, m}\left\langle c_{n}\left|L_{j}^{\dagger}\right| c_{m}\right\rangle\left\langle c_{m}\left|L_{j}\right| c_{n}\right\rangle \\
& +\left\langle c_{n}\left|J_{j}^{\dagger}\right| c_{m}\right\rangle\left\langle c_{m}\left|J_{j}\right| c_{n}\right\rangle=0,
\end{aligned}
$$

which proves Eq. (A13). The non-negativity conditions for $\mathcal{N}_{n m}$ (for $m \neq n$ ) and $\mathcal{T}_{m n}$ (for any $m, n$ ) hold trivially. Together with Eq. (A13) they also imply that $\mathcal{N}_{m n} \leq 0$ for $m=n$.

\section{APPENDIX B: DELAY FUNCTIONS, ACCURACY, AND IID SEQUENCES}

The appendix is structured as follows. In Appendix B 1, we define a delay function, that characterizes the probability distribution, with respect to time, of when an event occurs. This will eventually be applied to the ticks of the clock. We define the moments of the delay function, and introduce the accuracy $R$. We discuss the special case of a convolution of delay functions and the scaling of the accuracy in this case (Remark 6). Finally, in Appendix B 2, we prove a number of important lemmas concerning the accuracy, including one for sequences (i.e., convolutions) of delay functions (Lemma 2), and another for mixtures (Lemma 4).

\section{Delay functions: Definition, and behavior of the accuracy}

Definition 5: By a delay function, we refer to a nonnegative integrable function of time $t \geq 0, \tau: \mathbb{R}_{0}^{+} \longrightarrow$ $\mathbb{R}_{0}^{+}$, that is normalized or subnormalized,

$$
\int_{0}^{\infty} \tau(t) d t=Q \leq 1 .
$$

Definition 6: We define the mean (first moment), second moment, and variance of a delay function with respect to the normalized version of the delay function. That is, given a delay function $\tau(t)$ that integrates to $Q$ [see. $E q$. (B1)], the moments are calculated from $\tau(t) / Q$, which is a normalized probability distribution,

$$
\begin{aligned}
& Q=\left\langle t^{0}\right\rangle=\int_{0}^{\infty} \tau(t) d t, \\
& \mu=\frac{\left\langle t^{1}\right\rangle}{Q}=\int_{0}^{\infty} t \frac{\tau(t)}{Q} d t, \\
& \chi=\frac{\left\langle t^{2}\right\rangle}{Q}=\int_{0}^{\infty} t^{2} \frac{\tau(t)}{Q} d t,
\end{aligned}
$$

while the variance, denoted by $\sigma$, is defined in the usual manner,

$$
\sigma=\sqrt{\chi-\mu^{2}} .
$$

Remark 1: Note that the first and second moments may diverge.

Definition 7: The accuracy of a delay function $\tau(t)$ is defined by

$$
R[\tau]=\frac{\mu^{2}}{\sigma^{2}},
$$

if the first moment $\mu$ of the delay function is finite, and $R=0$ if it diverges. 
For simplicity of expression, we often omit the functional notation $R[\cdot]$, referring to the accuracy by simply $R$, augmented with a subscript or superscript when necessary.

Remark 2: This definition is discussed in the main text (Sec. II E), in the context of sequences of independent events all described by the same delay function. There we see that the ratio above is the average number of events before the uncertainty in the occurrence of the next event equals the average interval between events. For a discussion of the same, see Appendix E 2 a.

Remark 3: There are delay functions of arguably high precision, whose accuracy is not reflected well by $R$ [49]. Consider, for example, the following pathological [50] delay functions parametrized by $\epsilon>0$,

$$
\tau(t)=(1-\epsilon) \delta(t-1)+\epsilon \delta\left(t-1 / \epsilon^{2}\right),
$$

where $\delta(x)$ is the Dirac delta function. This delay function corresponds to a large probability of an event occurring at precisely $t=1$, and a small probability $\epsilon$ of the event occurring much later, at $t=1 / \epsilon^{2}$. For small $\epsilon$, the accuracy $R$ is of the order of $\epsilon$, and goes to zero in the limit $\epsilon \rightarrow 0$, even though the limiting delay function, $\delta(t-1)$, is very accurate. Other notions of accuracy, such as the operational number of alternate ticks " $N$ " [6] may be better suited to characterize these types of delay functions. The precise relationship between $R$ and $N$ is dealt with in detail in Ref. [49] (from which this delay function is sourced).
Remark 4: (The accuracy and $\gamma$ value). The accuracy can alternatively be expressed as

$$
R=\frac{1}{\frac{\chi}{\mu^{2}}-1}=\frac{1}{\gamma-1}, \quad \text { where } \quad \gamma=\frac{\chi}{\mu^{2}} .
$$

$\gamma$ has been introduced because it is usually easier to deal with than $R$, being the ratio between two moments of the delay function. Note that the relationship is bijective, and inverted (the smaller $\gamma$ is, the higher the value of $R$ ). The range of the accuracy is $R \in[0, \infty)$, corresponding to $\gamma \in$ $(1, \infty)$.

Remark 5: (The accuracy is invariant under renormalization). As the accuracy $R$ is defined via the first and second moments of the normalized version of the delay function, changing the normalization of $\tau(t)$ by multiplying it by a positive constant does not affect $R$.

\section{a. Combining delay functions in sequence-convolution}

Consider an arbitrary sequence of delay functions $\left\{\tau_{i}\right\}_{i=1}^{M}$. Then the following convolution of a subsequence

$$
\begin{aligned}
\tau^{(m)}(t)= & \int_{0}^{t} d t_{m-1} \iint \cdots \int_{0}^{t_{3}} d t_{2} \int_{0}^{t_{2}} d t_{1} \tau_{1}\left(t_{1}\right) \tau_{2}\left(t_{2}-t_{1}\right) \\
& \times \tau_{3}\left(t_{3}-t_{2}\right) \cdots \tau_{m}\left(t-t_{m-1}\right) \\
= & \left(\tau_{1} * \tau_{2} * \cdots * \tau_{m}\right)(t),
\end{aligned}
$$

where $*$ denotes convolution, is also a delay function. To see this, note that the integrand above is always non-negative, and thus $\tau^{(m)}$ is also non-negative. Furthermore, one may calculate, by direct integration, that the moments of $\tau^{(m)}$ are given by

$$
\begin{aligned}
& Q^{(m)}=\left\langle t^{0}\right\rangle=\prod_{i=1}^{m} Q_{i}, \\
& \text { where } Q_{i}=\int_{0}^{\infty} \tau_{i}(t) d t \text {, } \\
& \mu^{(m)}=\frac{\left\langle t^{1}\right\rangle}{Q^{(m)}}=\sum_{i=0}^{m} \mu_{i}, \\
& \text { where } \mu_{i}=\frac{1}{Q_{i}} \int_{0}^{\infty} t \cdot \tau_{i}(t) d t \text {, } \\
& \text { and } \quad \chi^{(m)}=\frac{\left\langle t^{2}\right\rangle}{Q^{(m)}}=\left(\sum_{i=0}^{m} \chi_{i}+\sum_{\substack{i, j=0 \\
i \neq j}}^{m} \mu_{i} \mu_{j}\right) \text {, } \\
& \text { where } \chi_{i}=\frac{1}{Q_{i}} \int_{0}^{\infty} t^{2} \cdot \tau_{i}(t) d t \text {. }
\end{aligned}
$$

Since each $Q_{i}$ is within [0,1], it follows that $Q^{(m)}$ is as well, and thus $\tau^{(m)}$ is a delay function (see Definition 5). Note that $\left\{\mu^{(m)}, \chi^{(m)}\right\}$ refer to the moments of the normalized version of the delay function (see Definition 6), and are finite if and only if every one of the corresponding moments of the individual delay functions do not diverge. 
Remark 6: A convolution of delay functions describes the case of a sequence of independent distributed events, and in the particular case of clocks, corresponds to reset clocks, those that go to a fixed state after ticking. In this case, each tick of the clock has an identical delay function with respect to the previous tick (all of the $\tau_{i}$ are the same and equal to $\tau_{1}$ ) and one can use Eq. (B8) to calculate the moments, and thus the accuracy of the $m^{\text {th }}$ tick,

$$
\begin{aligned}
& Q^{(m)}=Q_{1}^{m}, \\
& \mu^{(m)}=m \mu_{1}, \\
& \chi^{(m)}=m \chi+m(m-1) \mu^{2}, \\
& \sigma^{(m)}=\sqrt{m} \sigma_{1},
\end{aligned}
$$

from which we find that the accuracy $R^{(m)}=m R_{1}$.

\section{Lemmas on the accuracy of sequences, mixtures, and scaled delay functions}

Lemma 2: (The accuracy of a convolution of delay functions is limited by the sum of the accuracies.) If a delay function $\tau^{(m)}$ is formed out of the convolution of a sequence of delay functions as in Eq. (B7), then its accuracy $R\left[\tau^{(m)}\right]$ (Definition 7) is upper bounded by the sum of the accuracies of the individual delay functions that form the sequence, i.e.,

$$
R\left[\tau^{(m)}\right] \leq \sum_{i=1}^{m} R_{i},
$$

where $R_{i}$ is the accuracy of the $i$ th delay function $\tau_{i}$ in the sequence. Furthermore, this optimal accuracy is achieved if and only if the individual delay functions satisfy

$$
\frac{\mu_{i}}{R_{i}}=\frac{\mu_{j}}{R_{j}} \quad \forall i, j \in\{1,2, \ldots, m\},
$$

or equivalently, that they satisfy

$$
\frac{\sigma_{i}^{2}}{\mu_{i}}=\frac{\sigma_{j}^{2}}{\mu_{j}} \quad \forall i, j \in\{1,2, \ldots, m\},
$$

where $\mu_{i}$ and $\sigma_{i}$ are the mean and variance of the ith delay function, Eq. (B8).

Proof. We prove the lemma by induction. Consider that we express the delay function of the convolution as

$$
\begin{aligned}
\tau^{(m)} & =\tau^{(m-1)} * \tau_{m}, \\
\text { where } \quad \tau^{(m-1)} & =\tau_{1} * \tau_{2} * \cdots * \tau_{m-1} .
\end{aligned}
$$

Calculating the moments of $\tau^{(m)}$ with respect to the above subdivision, via Eq. (B8), we get

$$
\begin{aligned}
\mu^{(m)}= & \mu^{(m-1)}+\mu_{m}, \\
\chi^{(m)}= & \chi^{(m-1)}+\chi_{m}+2 \mu^{(m-1)} \mu_{m} \\
= & \left(\frac{1}{R^{(m-1)}}+1\right)\left[\mu^{(m-1)}\right]^{2}+\left(\frac{1}{R_{m}}+1\right) \mu_{m}^{2} \\
& +2 \mu^{(m-1)} \mu_{m},
\end{aligned}
$$

where we use Eq. (B6) to re-express the second moments $\chi$ with respect to the accuracies $R$.

Calculating the accuracy for $\tau^{(m)}$, using Eq. (B6) again,

$$
\begin{aligned}
R^{(m)} & =\frac{1}{\frac{\chi}{\mu^{2}}-1} \\
& =\left(1+\frac{\mu^{(m-1)}}{\mu_{m}}\right)^{2}\left[\frac{1}{R_{m}}+\frac{1}{R^{(m-1)}}\left(\frac{\mu^{(m-1)}}{\mu_{m}}\right)^{2}\right]^{-1} .
\end{aligned}
$$

To find the optimal accuracy $R^{(m)}$ given the subaccuracies $R^{(m-1)}, R_{m}$, we optimize the above expression with respect to the ratio of means $\mu^{(m-1)} / \mu_{m}$, which lies in $(0, \infty)$. At the limit points of the ratio, when $\mu^{(m-1)} / \mu_{m} \rightarrow 0$, one recovers $R^{(m)} \rightarrow R_{m}$, whereas when $\mu^{(m-1)} / \mu_{m} \rightarrow \infty$, one recovers $R^{(m)} \rightarrow R^{(m-1)}$.

In between, there is a single extremal (maximum) value, found by differentiating with respect to $\mu^{(m-1)} / \mu_{m}$, corresponding to

$$
R^{(m)}=R^{(m-1)}+R_{m},
$$

when the means satisfy

$$
\frac{\mu^{(m-1)}}{R^{(m-1)}}=\frac{\mu_{m}}{R_{m}}=\frac{\mu^{(m-1)}+\mu_{m}}{R^{(m-1)}+R_{m}}=\frac{\mu^{(m)}}{R^{(m)}} .
$$

Thus in general $R^{(m)} \leq R^{(m-1)}+R_{m}$.

One continues by induction, maximizing the accuracy of $R^{(m-1)}$ by splitting $\tau^{(m-1)}$ into the convolution of $\tau^{(m-2)}$ and $\tau_{m-1}$. Analogously to the above, one obtains that

$$
R^{(m)} \leq R^{(m-2)}+R_{m-1}+R_{m},
$$

with equality if and only if

$$
\frac{\mu^{(m-2)}}{R^{(m-2)}}=\frac{\mu_{m-1}}{R_{m-1}}=\frac{\mu_{m}}{R_{m}} .
$$

Proceeding in the same manner, one recovers the lemma. 
Definition 8: We define the partial norm $P_{t}[\tau]$ of a delay function $\tau$ (Definition 5) to be the finite integral

$$
P_{t}[\tau]=\int_{0}^{t} \tau\left(t^{\prime}\right) d t^{\prime}
$$

Thus $P_{t}[\tau] \leq Q \leq 1$ for all $t$.

Lemma 3: Given a delay function $\tau^{(m)}$ that is a convolution of a sequence of delay functions [Eq. (B7)], the partial norm (Definition 8) of $\tau^{(m)}$ is upper bounded by the products of the corresponding partial norms of the individual delay functions in the sequence, i.e.,

$$
P_{t}\left[\tau^{(m)}\right] \leq \prod_{i=0}^{m} P_{t}\left[\tau_{i}\right]
$$

Proof. Proof by induction. For a sequence of a single delay function, the statement of the lemma holds trivially (and is an equality). Next consider that the statement is proven for a sequence of $m$ arbitrary delay functions. Appending a single delay function $\tau_{m+1}$, we have that the convolution and its partial norm are given by

$$
\begin{aligned}
\tau^{(m+1)}(t) & =\left(\tau^{(m)} * \tau_{m+1}\right)(t), \\
P_{t}\left[\tau^{(m+1)}\right] & =\int_{0}^{t}\left(\int_{0}^{t^{\prime}} \tau_{m+1}\left(t^{\prime}-t^{\prime \prime}\right) \tau^{(m)}\left(t^{\prime \prime}\right) d t^{\prime \prime}\right) d t^{\prime} .
\end{aligned}
$$

In the integral above, the argument of $\tau^{(m)}$ runs within the interval $\left[0, t^{\prime}\right]$, which is contained in $[0, t]$, as $t^{\prime}$ itself runs within $[0, t]$. Furthermore, the argument of $\tau_{m+1}$, which is $\left(t^{\prime}-t^{\prime \prime}\right)$, is also constrained to be within the interval $[0, t]$. Thus we can upper bound the above integral by

$$
\begin{aligned}
P_{t}\left[\tau^{(m+1)}\right] & \leq \int_{0}^{t} \int_{0}^{t} \tau_{m+1}(x) \tau^{(m)}(y) d y d x, \\
\therefore P_{t}\left[\tau^{(m+1)}\right] & \leq P_{t}\left[\tau_{m+1}\right] P_{t}\left[\tau^{(m)}\right] \\
& \leq P_{t}\left[\tau_{m+1}\right] \prod_{i=0}^{m} P_{t}\left[\tau_{i}\right]=\prod_{i=0}^{m+1} P_{t}\left[\tau_{i}\right] .
\end{aligned}
$$

\section{a. Combining delay functions in mixtures}

Another manner in which one can combine delay functions is by mixing them in a convex combination.

Lemma 4: (The accuracy of a mixture is bounded by the best accuracy from among its components). Let a delay function be given by a sum of delay functions,

$$
\tau(t)=\sum_{i=1}^{m} \tau_{i}(t)
$$

where each $\tau_{i}$ is a subnormalized delay function (with nonzero zeroth moment), and the sum is also either normalized or subnormalized. Then the accuracy $R$ of the mixture is upper bounded by

$$
R[\tau] \leq \max _{i} R_{i}
$$

where $R_{i}$ is the accuracy of the ith delay function $\tau_{i}$. Furthermore, this inequality is only saturated in the case that every delay function $\tau_{i}$ has the same mean and accuracy, i.e.,

$$
\mu_{i}=\mu_{j} \quad \text { and } \quad R_{i}=R_{j} \quad \forall i, j \in\{1,2, \ldots, m\} .
$$

Proof. We prove the statement by induction. Split the set of delay functions that comprise the mixture into two subsets, and label the partial sums as $\tau^{(1)}(t)=\sum_{i=1}^{k} \tau_{i}(t)$ and $\tau^{(2)}(t)=\sum_{i=k+1}^{m} \tau_{i}(t)$, and thus

$$
\tau(t)=\tau^{(1)}(t)+\tau^{(2)}(t)
$$

We label the zeroth and (normalized) first moments of the two delay functions as $\left\{Q^{(1)}, \mu^{(1)}\right\}$ and $\left\{Q^{(2)}, \mu^{(2)}\right\}$, respectively [Eq. (B2)], and take their respective $\gamma$ values [Eq. (B6)] to be $\left\{\gamma^{(1)}, \gamma^{(2)}\right\}$. Note that both $Q^{(1)}, Q^{(2)}>0$. Thus for the composite delay function, calculating the moments explicitly from Eq. (B2),

$$
\begin{aligned}
Q & =\left\langle t^{0}\right\rangle=Q^{(1)}+Q^{(2)}, \\
\mu & =\frac{\left\langle t^{1}\right\rangle}{Q}=\frac{Q^{(1)} \mu^{(1)}+Q^{(2)} \mu^{(2)}}{Q^{(1)}+Q^{(2)}}, \\
\chi=\frac{\left\langle t^{2}\right\rangle}{Q} & =\frac{Q^{(1)} \chi^{(1)}+Q^{(2)} \chi^{(2)}}{Q^{(1)}+Q^{(2)}} \\
& =\frac{Q^{(1)}\left[\mu^{(1)}\right]^{2} \gamma^{(1)}+Q^{(2)}\left[\mu^{(2)}\right]^{2} \gamma^{(2)}}{Q^{(1)}+Q^{(2)}} .
\end{aligned}
$$

Calculating the $\gamma$ value of the composite delay function,

$$
\gamma=\left(Q^{(1)}+Q^{(2)}\right) \frac{Q^{(1)}\left[\mu^{(1)}\right]^{2} \gamma^{(1)}+Q^{(2)}\left[\mu^{(2)}\right]^{2} \gamma^{(2)}}{\left(Q^{(1)} \mu^{(1)}+Q^{(2)} \mu^{(2)}\right)^{2}} .
$$


Denote $q^{(1)}=Q^{(1)} /\left(Q^{(1)}+Q^{(2)}\right)$ and $q^{(2)}=Q^{(2)} /\left(Q^{(1)}+\right.$ $\left.Q^{(2)}\right)$, so that $q^{(1)}, q^{(2)}>0$ and $q^{(1)}+q^{(2)}=1$,

$$
\begin{aligned}
\gamma & =\frac{q^{(1)}\left[\mu^{(1)}\right]^{2} \gamma^{(1)}+q^{(2)}\left[\mu^{(2)}\right]^{2} \gamma^{(2)}}{\left(q^{(1)} \mu^{(1)}+q^{(2)} \mu^{(2)}\right)^{2}} \\
& \geq \frac{q^{(1)}\left[\mu^{(1)}\right]^{2} \gamma^{(1)}+q^{(2)}\left[\mu^{(2)}\right]^{2} \gamma^{(2)}}{q^{(1)}\left[\mu^{(1)}\right]^{2}+q^{(2)}\left[\mu^{(2)}\right]^{2}}
\end{aligned}
$$

by the convexity of the square. Next, denote $p^{(1)}=$ $q^{(1)}\left[\mu^{(1)}\right]^{2} /\left(q^{(1)}\left[\mu^{(1)}\right]^{2}+q^{(2)}\left[\mu^{(2)}\right]^{2}\right) \quad$ and $\quad p^{(2)}=q^{(2)}$ $\left[\mu^{(2)}\right]^{2} /\left(q^{(1)}\left[\mu^{(1)}\right]^{2}+q^{(2)}\left[\mu^{(2)}\right]^{2}\right)$, so that $p^{(1)}, p^{(2)}>0$ and $p^{(1)}+p^{(2)}=1$,

$$
\gamma \geq p^{(1)} \gamma^{(1)}+p^{(2)} \gamma^{(2)} \geq \min \left\{\gamma^{(1)}, \gamma^{(2)}\right\}
$$

As $\gamma$ is inversely related to $R$ [Eq. (B6)], it follows that

$$
R \leq \max \left\{R^{(1)}, R^{(2)}\right\}
$$

To saturate the upper bound, note that the inequality in Eq. (B41) is only an equality if

$$
\left(q^{(1)} \mu^{(1)}+q^{(2)} \mu^{(2)}\right)^{2}=q^{(1)}\left[\mu^{(1)}\right]^{2}+q^{(2)}\left[\mu^{(2)}\right]^{2},
$$

which by the strict convexity of the square function, is only satisfied when $\mu^{(1)}=\mu^{(2)}$. In this case, one has that

$$
\gamma=q^{(1)} \gamma^{(1)}+q^{(2)} \gamma^{(2)},
$$

which is equal to the minimum from among $\left\{\gamma^{(1)}, \gamma^{(2)}\right\}$ if and only if $\gamma^{(1)}=\gamma^{(2)}$. Thus to saturate the upper bound, both the mean and the accuracy of both delay functions must be equal, in which case, the mixture has the same mean and accuracy.

Proceeding by further splitting $\tau^{(1)}$ and $\tau^{(2)}$ until one recovers the original mixture, one arrives at the statement of the lemma.

\section{b. Scaling the timescale of a delay function- the invariance of the accuracy}

Lemma 5: If $\tau(t)$ is a delay function (Definition 5), then so is

$$
\tau^{\prime}(t)=a \tau(a t), \quad a>0 .
$$

Furthermore, the zeroth moment of $\tau^{\prime}$ is the same as that of $\tau$, the (normalized) first and second moments [Eq. (B2)] scale as $\left\{\mu / a, \chi / a^{2}\right\}$, and the accuracy $R$ is left unchanged.
Proof. Since $a>0, \tau^{\prime}(t)$ is a non-negative function. Calculating the zeroth moment,

$$
\begin{aligned}
Q^{\prime} & =\int_{0}^{\infty} a \tau(a t) d t \\
& =\int_{0}^{\infty} \tau(s) d s, \quad \text { where } s=a t \\
& =Q \leq 1 .
\end{aligned}
$$

Thus $\tau^{\prime}$ is also a delay function. For arbitrary moments, via the same change of variable, one finds that

$$
\frac{\left\langle t^{n}\right\rangle^{\prime}}{Q^{\prime}}=\frac{1}{a^{n}} \frac{\left\langle t^{n}\right\rangle}{Q}
$$

Thus $\mu^{\prime}=\mu / a$ and $\chi^{\prime}=\chi / a^{2}$. Finally, with respect to the $\gamma$ value [Eq. (B6)],

$$
\gamma^{\prime}=\frac{\chi^{\prime}}{\mu^{\prime 2}}=\frac{\chi / a^{2}}{(\mu / a)^{2}}=\frac{\chi}{\mu^{2}}=\gamma,
$$

from which it follows that the accuracy $R^{\prime}=R$.

Remark 7: The above lemma implies that the accuracy is independent of how quickly the event takes place, as would be measured by its frequency and resolution. In other words, the accuracy of a delay function is measured with respect to the natural timescale of the delay function itself, rather than an external reference.

\section{APPENDIX C: AN OPTIMAL CLASSICAL CLOCK-THE LADDER CLOCK}

\section{The ladder clock achieves accuracy $R_{j}=\boldsymbol{j} d$}

Here we discuss a simple classical clock that saturates the upper bound for the accuracy $R$ of classical clocks, and as far as we know, is the only one to do so. This is the classical clock used in Fig. 3(a). This clock, more precisely, the discrete version of the continuous clock we discuss below, was introduced in Ref. [13]. There it was shown to perform with a similar linear scaling in the dimension $d$, but for the alternative definition of accuracy via the alternate ticks game [6]. This optimal clock may also be approached thermodynamically as in Ref. [9], in the limit of semiclassical dynamics, and of infinite entropy cost.

As discussed in Corollary 1, a classical clock is completely specified by a pair $\mathcal{N}, \mathcal{T} \in \mathbb{R}^{(d \times d)}$ of stochastic generators and the initial state of the clock, $V_{0} \in \mathcal{P}_{d}$. In 
the case of the ladder clock, these are

$$
\begin{aligned}
\mathcal{N} & =\left[\begin{array}{ccccccc}
-1 & 0 & \ldots & & & \ldots & 0 \\
1 & -1 & 0 & \ldots & & & \vdots \\
0 & 1 & -1 & 0 & \ldots & & \\
\vdots & \ddots & \ddots & \ddots & \ddots & & \\
& & \ddots & \ddots & \ddots & \ddots & \vdots \\
\vdots & & & 0 & 1 & -1 & 0 \\
0 & \ldots & & \ldots & 0 & 1 & -1
\end{array}\right], \\
{[\mathcal{T}]_{i, j} } & = \begin{cases}1 & \text { if } i=1, j=d, \\
0 & \text { otherwise, }\end{cases}
\end{aligned}
$$

and the initial state is chosen to be the vector of probabilities $\left[V_{0}(0)\right]_{i}=\delta_{i, 1}$. Physically, the choice of tick generator means that we can only tick from the $d^{\text {th }}$ site, while the choice of initial state $V_{0}(0)$ means that the clock starts out with all its population on the first site. Furthermore, $\mathcal{N}$ generates movement from the basis state $i$ to the basis state $i+1$, and thus we call this clock the ladder clock.

As the tick generator $\mathcal{T}$ is rank-1, it follows that the clock is a reset clock (Definition 2). Each tick is thus identical and independent of the others, and the delay function of the $n$th tick of the clock is just the convolution of that of the first tick with itself $n$ times (for more details, see Appendix E, and specifically Appendix a). From Remark 6 , we conclude that the accuracy of the $n$th tick is $n R_{1}$, where $R_{1}$ is the accuracy of a single tick.

Furthermore, one can understand a single tick itself as a sequence of $d$ identical and independent events, that of moving from site 1 to site 2, 2 to 3 , and so on, with the final event being the tick moving the state from site $d$ back to site 1 . The accuracy of a single tick is thus $d$ times the accuracy of the delay function of a single site jump, which turns out to be $R=1$. In turn, the accuracy of a single tick of the clock is $R_{1}=d$, and that of the $n$th tick is $R_{n}=n d$.

We proceed to prove these statements from the analytical form of the clock state and delay function of the tick.

\section{The time evolution of the ladder clock}

The unnormalized state of the ladder clock corresponding to the case in which no tick is generated during the time interval $[0, t]$ is given by the equation (for a more detailed discussion on this, see Sec. E)

$$
\begin{gathered}
V_{0}(t)=e^{t \mathcal{N}} V_{0}(0)=e^{-t}\left[\begin{array}{ccccccccc}
1 & 0 & 0 & \ldots & & & & \ldots & 0 \\
f_{2}(t) & 1 & 0 & 0 & & & & & \vdots \\
f_{3}(t) & f_{2}(t) & 1 & 0 & 0 & & & & \\
\vdots & f_{3}(t) & f_{2}(t) & 1 & 0 & 0 & & & \\
& & \ddots & \ddots & \ddots & \ddots & \ddots & & \\
& & & f_{3}(t) & f_{2}(t) & 1 & 0 & 0 & \vdots \\
& & & & f_{3}(t) & f_{2}(t) & 1 & 0 & 0 \\
\vdots & & & & & f_{3}(t) & f_{2}(t) & 1 & 0 \\
f_{d}(t) & \ldots & & & & \cdots & f_{3}(t) & f_{2}(t) & 1
\end{array}\right]\left[\begin{array}{c}
1 \\
0 \\
\vdots \\
\vdots \\
\vdots \\
f_{2}(t) \\
f_{3}(t) \\
\vdots \\
\vdots \\
\vdots \\
\vdots \\
\vdots \\
f_{d}(t)
\end{array}\right], \quad f_{k}(t)=\frac{t^{k-1}}{(k-1) !} \\
=e^{-t}
\end{gathered}
$$


A direct calculation of the mean $\mu(t)$ and standard deviation $\sigma(t)$ associated with the population at time $t$ in the limit $0 \leq t \ll d$, using Eq. (C2) yields

$$
\begin{aligned}
\mu(t) & =\sum_{n=1}^{d} n\left[V_{0}(t)\right]_{n}, \\
\sigma(t) & =\sqrt{\sum_{n=1}^{d}[n-\mu(t)]^{2}\left[V_{0}(t)\right]_{n} .}
\end{aligned}
$$

From these expressions we see that

$$
\begin{aligned}
& \mu(t) \approx \sum_{n=1}^{\infty} n\left[V_{0}(t)\right]_{n}=t+1, \\
& \sigma(t) \approx \sqrt{\sum_{n=1}^{\infty}[n-\mu(t)]^{2}\left[V_{0}(t)\right]_{n}}=\sqrt{t} .
\end{aligned}
$$

In other words, the mean is approximately proportional to the distance traveled by the initial state, while the standard deviation is proportional to the square root of the distance traveled by the initial state. Physically, the reason why this is only approximately true for short times, is because when population reaches the last site $n=d$, the population is "lost" from the no-tick space due to a tick happening.

From Eqs. (C1) and (C2), it follows that the delay function associated with the first tick, $P_{T_{1}}(t)$ (defined in Sec. II E) is

$$
P_{T_{1}}(t)=\left\|\mathcal{T} V_{0}(t)\right\|_{1}=e^{-t} \frac{t^{d-1}}{(d-1) !} .
$$

A direct calculation of the mean $\mu_{1}$, standard deviation $\sigma_{1}$, and accuracy $R_{1}$; for these classical clocks according to the delay function Eq. (C6), yields

$$
\mu_{1}=d, \quad \sigma_{1}=\sqrt{d}, \quad R_{1}=\frac{\mu_{1}^{2}}{\sigma_{1}^{2}}=d .
$$

Since the ladder clock is a reset clock, it follows (see Sec. E for a proof) that the accuracy of the $j^{\text {th }}$ tick is $R_{j}=j R_{1}$, and thus this completes the proof of Eq. (33) in Theorem 1.

\section{APPENDIX D: CLASSICALLY GENERATED MARKOVIAN SEQUENCES}

In this appendix we discuss Markovian dynamics of classical systems. We begin in Appendix D 1 by discussing classical finite-dimensional states, the differential equations that govern the generation of events, and their associated delay functions. We demonstrate the properties of the dynamical generators that we require later in the proof. In Appendix D 2 we discuss sequences of events generated by Markovian dynamics, and some of their properties, continuing in Appendix D 3 to differentiate between general sequences of events and independent sequences, those in which each event is independent of the dynamics of the prior event. Finally in Appendix D 4, we prove that the accuracy of general sequences is upper bound by that of independent sequences, a result that will translate directly to the case of clocks, in which case reset clocks will upper bound the accuracy of general clocks.

\section{Events generated by classical (stochastic) dynamics}

\section{a. Classical states are population vectors}

Here we define the state space for classical Markovian dynamics, of which clocks are a special case. In the main text (Sec. II D), we argued that classical clocks, which are a special case of quantum clocks restricted to a fixed orthonormal basis, may be described by stochastic dynamics on the vector of diagonal elements of the quantum state with respect to the restricted basis. In light of this description, we define classical states accordingly.

Definition 9: The canonical basis is a preferred orthonormal basis $\left\{\mathbf{e}_{i}\right\}_{i=0}^{d}$ for a real vector space of finite dimension $d$. Each $\mathbf{e}_{i}$ is referred to as a canonical state.

Definition 10: A classical state, or population vector, is a vector $v \in \mathbb{R}^{d}$ all of whose elements with respect to the canonical basis (Definition 9) are greater or equal to zero, and whose sum of the elements in the canonical basis is less or equal to one. Thus $v=\sum_{i=1}^{d} v_{i} \mathbf{e}_{i}$ is a population vector if and only if

$$
\begin{aligned}
v_{i} & \geq 0 \quad \forall i, \\
\text { and } \quad \sum_{i} v_{i} & \leq 1 .
\end{aligned}
$$

Remark 8: In this Appendix, we deal only with classical states, and so omit the prefix classical for simplicity.

Definition 11: For this Appendix, we define the e sum (that stands for "element sum") of a real vector $v \in \mathbb{R}^{d}$ as the sum of its elements with respect to the canonical basis (Definition 9). Thus if $v=\sum_{i=1}^{d} v_{i} \mathbf{e}_{i}$, then

$$
\|v\|_{\Sigma}=\sum_{i=1}^{d} v_{i} .
$$

Remark 9: Note that for population vectors, the e sum coincides with the 1-norm, as all of the elements in said basis are non-negative. We use the simple sum because we deal with negative vectors as well, and the e sum has the advantage of commuting with other finite sums and finite integrals. 


\section{b. Generators of stochastic (Markovian) dynamics}

From standard probability theory, the differential equation for Markovian dynamics is given by

$$
\frac{d}{d t} V(t)=\hat{M} V(t)
$$

where $V(t)$ is the state at time $t$ and $\hat{M}$ is a timeindependent linear operator, referred to as the Kolmogorov generator of forward dynamics. If the dynamics are also responsible for the generation of events, then the generator $\hat{M}$ is split into

$$
\hat{M}=\mathcal{N}+\mathcal{T},
$$

where $\mathcal{T}$ is associated with the generation of events, and the probability density (probability per unit time) that the event occurs at a time $t$ is given by

$$
P(t)=\|\mathcal{T} V(t)\|_{\Sigma} .
$$

We proceed to discuss the precise properties of these generators that we require in the proof.

Definition 12: By a pair of stochastic generators, we mean a pair of linear operators $\{\mathcal{N}, \mathcal{T}$ \}on a finitedimensional real-valued vector space $\mathbb{R}^{d}$, that we refer to as the nonevent generator and event generator, respectively, such that the following conditions are satisfied:

(a) $\mathcal{N}$ is an endomorphism, i.e., it takes vectors from $\mathbb{R}^{d}$ to $\mathbb{R}^{d}$, and when expressed as a matrix with respect to the canonical basis,

$$
\mathcal{N}_{i j} \begin{cases}\leq 0 & \text { for } i=j, \\ \geq 0 & \text { for } i \neq j .\end{cases}
$$

(b) $\mathcal{T}$ takes vectors from $\mathbb{R}^{d}$ to a real vector space of possibly different dimension $\mathbb{R}^{d^{\prime}}$. Expressed as a matrix with respect to the canonical bases of both (input and output) spaces, $\mathcal{T}$ has $d^{\prime}$ rows and $d$ columns, and must be elementwise non-negative, i.e.,

$$
\mathcal{T}_{i j} \geq 0 \quad \forall i, j .
$$

(c) The pair of generators must satisfy

$$
\sum_{i=1}^{d^{\prime}} \mathcal{T}_{i j}+\sum_{i=1}^{d} \mathcal{N}_{i j} \leq 0 \quad \forall j .
$$

Remark 10: The non-negative nature of $\mathcal{T}$ follows from the requirement that the probability density of the event
[Eq. (D6)] must be non-negative, and the state following the event must also be a population vector. The offdiagonal elements of both $\mathcal{N}$ and $\mathcal{T}$ represent transition probabilities between different canonical states, and are therefore also non-negative. Finally, the condition on the column sums [Eq. (D9)] is necessary for the e-sum norm of the state to be nonincreasing, from which the condition on the diagonal elements of $\mathcal{N}$ follows.

Remark 11: We use the more general norm nonincreasing rather than norm-preserving generators in the Appendix because (1) the results apply to the more general case anyway, and (2) we require results on norm nonincreasing dynamics later in the proof.

Corollary 2: If $\{\mathcal{N}, \mathcal{T}\}$ are a pair of stochastic generators, then so is $\{\mathcal{N}, \hat{\mathbf{0}}\}$, where $\hat{\mathbf{0}}$ is the zero operator with the same input and output spaces as $\mathcal{T}$.

Proof. The zero operator clearly satisfies Eq. (D8). Furthermore, we verify that Eq. (D9) is satisfied for the new pair,

$$
\sum_{i=1}^{d} \mathcal{N}_{i j} \leq-\left(\sum_{i=1}^{d^{\prime}} \mathcal{T}_{i j}\right)<0 \quad \forall j
$$

as all of the elements of $\mathcal{T}$ are non-negative.

Remark 12: Given a linear endomorphism $\mathcal{N}$, we consider it to be a nonevent generator if it forms a pair of stochastic generators with the zero operator, which is equivalent to requiring it to for a pair of stochastic generators with at least one elementwise generator $\mathcal{T}$. On the other hand, every linear operator $\mathcal{T}$ that is elementwise non-negative with respect to the canonical bases for the input and output space is an event generator, as one can always find a nonevent generator with diagonal elements that are negative enough so that the two taken together form a pair of stochastic generators.

Corollary 3: If $\{\mathcal{N}, \mathcal{T}\}$ are a pair of stochastic generators (Definition 12) and $\mathcal{T}$ is an endomorphism, i.e., it takes vectors from $\mathbb{R}^{d}$ to $\mathbb{R}^{d}$, then the pair $\{\mathcal{N}+\mathcal{T}, \hat{\mathbf{0}}\}$ is also a pair of stochastic generators. Equivalently, $\mathcal{N}+\mathcal{T}$ is a nonevent generator.

Proof. Equation (D9) is trivially satisfied, while the zero operator by definition satisfies Eq. (D8). The off-diagonal elements of $\mathcal{N}$ and $\mathcal{T}$ are individually non-negative, and thus the same holds for the sum, satisfying the second condition in Eq. (D7). 
Finally, to verify the condition on the diagonal elements [first line of Eq. (D7)],

$$
\mathcal{N}_{j j}+\mathcal{T}_{j j}=\sum_{i=1}^{d}\left(\mathcal{N}_{i j}+\mathcal{T}_{i j}\right)-\sum_{i \neq j}^{d}\left(\mathcal{N}_{i j}+\mathcal{T}_{i j}\right)<0 \quad \forall j,
$$

as the first sum on the right is nonpositive by Eq. (D9), and the second (subtracted) sum comprises solely non-negative numbers, by Eqs. (D7) and (D8).

Corollary 4: If $\{\mathcal{N}, \mathcal{T}\}$ are a pair of stochastic generators, then so are all of the pairs $\left\{\mathcal{N}, \mathcal{T}_{j}\right\}$, where $\mathcal{T}_{j}$ is constructed by setting all of the rows in $\mathcal{T}$ to zero, save the $j$ th one.

Proof. Equation (D7) is satisfied trivially as the nonevent generator $\mathcal{N}$ is left unchanged. Setting rows to zero in $\mathcal{T}$ also maintains Eq. (D8). Finally, for Eq. (D9), for the $j^{\text {th }}$ column, the modified left-hand side of the expression is missing some nonzero elements from the original event generator $\mathcal{T}$, and is therefore less than or equal to the original expression, still satisfying the inequality.

Corollary 5: If $\{\mathcal{N}, \mathcal{T}\}$ are a pair of stochastic generators, then so is $\{a \mathcal{N}, a \mathcal{T}\}$, where $a>0$.

Proof. By direct substitution into Definition 12, one verifies that all of the inequalities are maintained as the scaling factor $a$ is positive.

Lemma 6: (From infinitesimal generators to finite-time transition matrices.) Given any stochastic nonevent generator $\mathcal{N}$, and a population vector $V$,

(a) $e^{\mathcal{N} t}$ is a transition matrix [51] for all $t \geq 0$, i.e., If $\mathcal{M}=e^{\mathcal{N} t}$, then

$$
\begin{aligned}
\mathcal{M}_{i j} & \geq 0 \quad \forall i, j, \\
\sum_{i} \mathcal{M}_{i j} & \leq 1 \quad \forall j .
\end{aligned}
$$

(b) $e^{\mathcal{N} t} V$ is a population vector (Definition 10) for all $t \geq 0$, and its norm (e sum) is nonincreasing in time.

Proof. One of the definitions of the exponential function is via

$$
e^{\mathcal{N} t}=\lim _{m \rightarrow \infty}\left(\mathbb{1}+\frac{\mathcal{N} t}{m}\right)^{m} .
$$

The matrix within the parentheses has non-negative offdiagonal entries since $\mathcal{N}_{i j}>0$ for $i \neq j$. Furthermore, for the diagonal entries, one can always pick $m$ large enough so that $\mathbb{1}+t \mathcal{N}_{i i} / m>0$, and thus, for large enough $m$, the above expression is a positive power of a matrix with nonnegative entries, which must be non-negative elementwise. Thus $e^{\mathcal{N} t}$ is elementwise non-negative.

To prove that the column sums of $\mathcal{M}$ are less or equal to 1 , we first prove the properties of $e^{\mathcal{N} t} V$. As $V$ is a population vector, all of its elements are non-negative, and thus the elements of $e^{\mathcal{N} t} V$ are also non-negative. Labeling this vector as $V(t)=\sum_{i} V_{i}(t) \mathbf{e}_{i}$ where each $V_{i}(t) \geq 0$, we may calculate the rate of change of its e sum (Definition 11),

$$
\begin{aligned}
\frac{d}{d t}\|V(t)\|_{\Sigma} & =\left\|\frac{d}{d t} e^{\mathcal{N} t} V\right\|_{\Sigma} \\
& =\|\mathcal{N} V(t)\|_{\Sigma} \\
& =\sum_{i j} \mathcal{N}_{i j} V_{j}(t) \\
& =\sum_{j}\left(\sum_{i} \mathcal{N}_{i j}\right) V_{j} \leq 0
\end{aligned}
$$

as each of the column sums of $\mathcal{N}$ is nonpositive [Eq. (D7)]. Thus $V(t)$ is also a population vector for all $t \geq 0$, with nonincreasing norm. Finally take $V=\mathbf{e}_{j}$. As the norm of $V(t)$ is less or equal to 1 for all $t \geq 0$, one has for the transition matrix $\mathcal{M}=e^{\mathcal{N} t}$ that

$$
\begin{aligned}
\|V(t)\|_{\Sigma} & =\left\|\mathcal{M} \mathbf{e}_{j}\right\|_{\Sigma} \\
& =\sum_{i} \mathcal{M}_{i j} \leq 1 .
\end{aligned}
$$

\section{Sequences of events}

Definition 13: By a Markovian sequence of events, we mean a sequence, finite or infinite, of time-dependent population vectors $\left\{V^{(n)}(t): \mathbb{R}^{+} \rightarrow \mathbb{R}^{d^{(n)}}\right\}$ together with a corresponding sequence of pairs of stochastic generators $\left\{\mathcal{N}^{(n)}: \mathbb{R}^{d^{(n)}} \rightarrow \mathbb{R}^{d^{(n)}}, \mathcal{T}^{(n)}: \mathbb{R}^{d^{(n)}} \rightarrow \mathbb{R}^{d^{(n+1)}}\right\}$, the dynamics of which are given by

$$
\frac{d}{d t} V^{(n)}(t)= \begin{cases}\mathcal{N}^{(0)} V^{(0)}(t) & \text { for } n=0, \\ \mathcal{N}^{(n)} V^{(n)}(t)+\mathcal{T}^{(n-1)} V^{(n-1)}(t) & \text { for } n \geq 1,\end{cases}
$$

and that satisfy the initial conditions

(a) $V^{(0)}(0)$ is a normalized population vector,

(b) $V^{(n)}(0)=\mathbf{0}$ for all $n \geq 1$. 
Furthermore, we define the delay function of the $n$th event as

$$
\tau^{(n)}(t)=\left\|\mathcal{T}^{(n-1)} V^{(n-1)}(t)\right\|_{\Sigma}
$$

for $n \geq 1$. For $n=0$, the delay function is $\tau^{(0)}(t)=\delta(t)$, the Dirac delta distribution.

Remark 13: Note that the fact that the $V^{(n)}$ are population vectors and that the $\tau^{(n)}$ are delay functions is not immediate from the above definition, but is nevertheless implied, as the following lemmas will establish.

Lemma 7: (Events in a Markovian sequence are recursive convolutions.) For a Markovian sequence of events (Definition 13), each vector in the sequence can be expressed as a convolution with respect to the previous one, i.e., for $n \geq 1$,

$$
V^{(n)}(t)=\int_{0}^{t} e^{\mathcal{N}^{(n)}\left(t-t^{\prime}\right)} \mathcal{T}^{(n-1)} V^{(n-1)}\left(t^{\prime}\right) d t^{\prime}
$$

and for $n=0$,

$$
V^{(0)}(t)=e^{\mathcal{N}^{(0)} t} V^{(0)}(0)
$$

Proof. Proof by induction. Consider we define the sequence $f^{(n)}(t)$ by

$$
f^{(n)}(t)= \begin{cases}e^{\mathcal{N}^{(0)} t} V^{(0)}(0) & \text { for } n=0 \\ \int_{0}^{t} e^{\mathcal{N}^{(n)}\left(t-t^{\prime}\right)} \mathcal{T}^{(n-1)} f^{(n-1)}\left(t^{\prime}\right) d t^{\prime} & \text { for } n \geq 1\end{cases}
$$

For $n=0$, by construction, $f^{(0)}(t)$ has the same initial conditions and obeys the same differential equation as $V^{(0)}(t)$, and is thus equal to $V^{(0)}(t)$.

Proceeding, let $f^{(n-1)}(t)=V^{(n-1)}(t)$ for some $n \geq 1$. Then differentiating $f^{(n)}(t)$ from Eq. (D24) using Leibniz's rule, we find that

$$
\begin{aligned}
\frac{d}{d t} f^{(n)}(t)= & \frac{d}{d t} \int_{0}^{t} e^{\mathcal{N}^{(n)}\left(t-t^{\prime}\right)} \mathcal{T}^{(n-1)} f^{(n-1)}\left(t^{\prime}\right) d t^{\prime} \\
= & \mathcal{N}^{(n)} \int_{0}^{t} e^{\mathcal{N}^{(n)}\left(t-t^{\prime}\right)} \mathcal{T}^{(n-1)} f^{(n-1)}\left(t^{\prime}\right) d t^{\prime} \\
& +\mathcal{T}^{(n-1)} f^{(n-1)}(t) \\
= & \mathcal{N}^{(n)} f^{(n)}(t)+\mathcal{T}^{(n-1)} V^{(n-1)}(t)
\end{aligned}
$$

and thus $f^{(n)}(t)$ obeys the same differential equation as $V^{(n)}(t)$. Their initial conditions are also the same as $f^{(n)}(0)=\mathbf{0}=V^{(n)}(0)$ for $n \geq 1$. Thus $f^{(n)}(t)=V^{(n)}(t)$, and the rest follows by induction.

Lemma 8: (States and delay functions of a Markovian sequence of events.) For a Markovian sequence of events (Definition 13),

(a) every vector $V^{(n)}(t)$ in the sequence is a population vector (Definition 10),

(b) every $\tau^{(n)}(t)$ is a delay function (Definition 5).

Proof. We first prove by induction that the elements of every $V^{(n)}(t)$ with respect to the canonical basis are nonnegative. At $t=0$, this is true by definition. Furthermore, by Lemma $7, V^{(0)}(t)$ evolves via the transition operator $e^{\mathcal{N}^{(0)} t}$, and is thus always non-negative, by Lemma 6 . Proceeding to $V^{(n)}(t)$ from Eq. (D22),

$$
V^{(n)}(t)=\int_{0}^{t} e^{\mathcal{N}^{(n)}\left(t-t^{\prime}\right)} \mathcal{T}^{(n-1)} V^{(n-1)}\left(t^{\prime}\right) d t^{\prime}
$$

note that if $V^{(n-1)}(t)$ is non-negative for all $t$, then so is $\mathcal{T}^{(n-1)} V^{(n-1)}(t)$ as the elements of $\mathcal{T}^{(n-1)}$ are all nonnegative (Definition 12), and since this is multiplied by another transition matrix (Lemma 6), whose elements are all non-negative, the integrand is non-negative, and thus $V^{(n)}(t)$ is also non-negative with respect to the canonical basis for $t \geq 0$.

To prove that each vector is a population vector, i.e., has an e sum (Definition 11) less than or equal to 1, we prove the statement for the sum of e sums $\sum_{n=0}^{m}\left\|V^{(n)}(t)\right\|_{\Sigma}, m \geq 0$, from which the weaker statement follows.

$$
\begin{aligned}
\frac{d}{d t} \sum_{n=0}^{m}\left\|V^{(n)}(t)\right\|_{\Sigma} & =\sum_{n=0}^{m}\left\|\frac{d}{d t} V^{(n)}(t)\right\|_{\Sigma} \\
& =\left\|\mathcal{N}^{(0)} V^{(0)}(t)\right\|_{\Sigma}+\sum_{n=1}^{m}\left(\left\|\mathcal{N}^{(n)} V^{(n)}(t)\right\|_{\Sigma}+\left\|\mathcal{T}^{(n-1)} V^{(n-1)}(t)\right\|_{\Sigma}\right)
\end{aligned}
$$




$$
\begin{aligned}
= & \sum_{n=0}^{m-1}\left(\left\|\mathcal{N}^{(n)} V^{(n)}(t)\right\|_{\Sigma}+\left\|\mathcal{T}^{(n)} V^{(n)}(t)\right\|_{\Sigma}\right)+\left\|\mathcal{N}^{(m)} V^{(m)}(t)\right\|_{\Sigma} \\
= & \sum_{n=0}^{m-1} \sum_{j=1}^{d^{(n)}}\left(\sum_{i=1}^{d^{(n)}}\left[\mathcal{N}^{(n)}\right]_{i j}+\sum_{i=1}^{d^{(n+1)}}\left[\mathcal{T}^{(n)}\right]_{i j}\right)\left[V^{(n)}(t)\right]_{j}+\sum_{j=1}^{d^{(m)}}\left(\sum_{i=1}^{d^{(m)}}\left[\mathcal{N}^{(m)}\right]_{i j}\right)\left[V^{(m)}(t)\right]_{j},
\end{aligned}
$$

where we use the definition of the e sum, Definition 11. The right-hand side of the above equation is nonpositive, as the two column sums in parentheses above are nonpositive (see Definition 12 and Corollary 2). Thus

$$
\frac{d}{d t}\left\|\sum_{n=0}^{m} V^{(n)}(t)\right\|_{\Sigma} \leq 0
$$

and thus the sum of the norms is nonincreasing. At $t=$ 0 the sum is 1 by definition (Definition 13), and thus the sum is less than or equal to unity for $t \geq 0$, from which it follows that each term in the sum, which is also nonnegative, must also be between 0 and 1 . Thus every $V^{(n)}$ is a population vector for all $t \geq 0$.

To prove that every $\tau^{(m)}$ is a delay function, note in Eq. (D21) that as $\mathcal{T}^{(m-1)}$ and $V^{(m-1)}$ are elementwise nonnegative and bounded, it follows that $\tau^{(m)}$ is non-negative and bounded as well. To prove integrability, we add up Eq. (D21) (with $n=m+1$ ) and Eq. (D32) to get

$$
\begin{aligned}
\tau^{(m+1)}(t)+\frac{d}{d t} \sum_{n=0}^{m}\left\|V^{(n)}(t)\right\|_{\Sigma} \\
=\sum_{n=0}^{m} \sum_{j=1}^{d^{(n)}}\left(\sum_{i=1}^{d^{(n)}}\left[\mathcal{N}^{(n)}\right]_{i j}+\sum_{i=1}^{d^{(n+1)}}\left[\mathcal{T}^{(n)}\right]_{i j}\right) \\
\quad \times\left[V^{(n)}(t)\right]_{j},
\end{aligned}
$$

where $m \geq 0$. The right-hand side is nonpositive from the properties of the column sums of the generators [Eq. (D9)], and we label it as the function $g(t)$. Thus $g(t) \leq 0$.

Integrating from $t=0$ to $t=T$, one obtains

$$
\begin{aligned}
& \int_{0}^{T} \tau^{(m+1)}(t) d t \\
& =-\int_{0}^{T} \frac{d}{d t} \sum_{n=0}^{m}\left\|V^{(n)}(t)\right\|_{\Sigma} d t+\int_{0}^{T} g(t) d t \\
& =\sum_{n=0}^{m}\left\|V^{(n)}(0)\right\|_{\Sigma}-\left\|\sum_{n=0}^{m} V^{(n)}(T)\right\|_{\Sigma}+\int_{0}^{T} g(t) d t \\
& =1-\sum_{n=0}^{m}\left\|V^{(n)}(T)\right\|_{\Sigma}+\int_{0}^{T} g(t) d t
\end{aligned}
$$

using the initial conditions for the sequence of states (Definition 13). As $g(t) \leq 0$ for all $t \geq 0$, the right-hand side of the above expression is less than or equal to 1 for all $T$. However, the integrand on the left-hand side is non-negative, and thus the integral is nondecreasing with respect to $T$. It follows from the monotone convergence theorem [52] that the $\operatorname{limit}_{\lim } \rightarrow \infty \int_{0}^{T} \tau^{(m+1)}(t) d t$ exists and is less than or equal to 1 .

Thus every $\tau^{(m+1)}$ for $m \geq 0$, or equivalently, every $\tau^{(m)}$ for $m \geq 1$, is a delay function.

Corollary 6: Given a single pair of stochastic generators $\{\mathcal{N}, \mathcal{T}\}$ acting on $\mathbb{R}^{d}$, together with an arbitrary population vector $V \in \mathbb{R}^{d}$, the following is a delay function:

$$
\tau(t)=\left\|\mathcal{T} e^{\mathcal{N} t} V\right\|_{\Sigma}
$$

Proof. Take an arbitrary Markovian sequence (Definition 13) with the choice of $V^{(0)}(0)=V, \mathcal{T}^{(0)}=\mathcal{T}$, and $\mathcal{N}^{(0)}=$ $\mathcal{N}$. Apply Lemma 8 to deduce that $\tau^{(1)}$ is a delay function, and substitute the solution for the time-evolved state $V^{(0)}(t)$ from Lemma 7 to the expression of $\tau^{(1)}(t)$ from Definition 13.

Lemma 9: (Invariance of the dynamics of an event with respect to the following event.) For any Markovian sequence of events, the state and delay function of the $n$th event in the sequence (as well as those of every previous event) is invariant with respect to a change in the $n$th event generator $\mathcal{T}^{(n)}$ that leaves its column sums unchanged, i.e., if $\mathcal{T}^{(n)^{\prime}}$ is chosen such that its matrix elements satisfy

$$
\sum_{i}\left[\mathcal{T}^{(n) \prime}\right]_{i j}=\sum_{i}\left[\mathcal{T}^{(n)}\right]_{i j} \quad \forall j
$$

Proof. From Lemma 7, it is clear that the state $V^{(n)}(t)$ corresponding to the $n$th event is independent of $\mathcal{T}^{(n)}$, and does not affect the states and delay functions of any of the events prior to the $n$ th. The independence of the $V^{(i)}(t)$ and $\tau^{(i)}(t)$, where $i<n$ follows from the fact that the initial conditions and differential equations for all of the $i<n$ are independent of $i \geq n$. 
As for the delay function of the $n$th event, from Eq. (D21),

$$
\begin{aligned}
\tau^{(n)}(t) & =\left\|\mathcal{T}^{(n-1)} V^{(n-1)}(t)\right\|_{\Sigma} \\
& =\sum_{i j}\left[\mathcal{T}^{(n-1)}\right]_{i j}\left[V^{(n-1)}(t)\right]_{j} \\
& =\sum_{j}\left(\sum_{i}\left[\mathcal{T}^{(n-1)}\right]_{i j}\right)\left[V^{(n-1)}(t)\right]_{j},
\end{aligned}
$$

and is thus invariant under any operation that leaves the column sums of $\mathcal{T}^{(n-1)}$ unchanged.

Remark 14: Lemma 9 reflects the fact that the tick generator $\mathcal{T}^{(n)}$ encodes both the amount of probability of the event being generated from each canonical state (as reflected by each of the column sums) as well as the state following the generation of an event (encoded in the individual elements of $\mathcal{T}^{(n)}$ ), the first of which affects the dynamics of the $n$th event, while the second affects only subsequent events.

\section{Independent sequences of events}

Here we discuss a special class of Markovian sequences, those in which the state of the system immediately following an event is a fixed state, invariant with respect to the state of the system prior to the event. Such sequences have useful properties that we use later in the proof.

Definition 14: A Markovian sequence of events (Definition 13) is called an independent Markovian sequence of events if every one of its event generators $\mathcal{T}^{(n)}$ is a rank-1 linear operator.

Remark 15: (Properties of an independent sequence.) If $\mathcal{T}$ is a rank-1 linear operator, then all of its columns are proportional to one nonzero column, and therefore, given any vector $V$ in the domain of $\mathcal{T}$, the product $\mathcal{T} V$ is also proportional to the same fixed column. For a Markovian sequence of events, the term in the dynamical equation $\mathcal{T}^{(n-1)} V^{(n-1)}(t)$ [see Eqs. (D20) and (D21)] represents the state following the occurrence of the $n$th event, and thus an independent sequence is one in which this product is proportional to some fixed state for all $t \geq 0$.

Definition 15: For an independent Markovian sequence of events (Definition 13), we define the sequence of event reset states $\left\{V_{R}^{(n)}\right\}$ as follows: for each $n, V_{R}^{(n)}$ is the unique normalized vector that every column of $\mathcal{T}^{(n)}$ is proportional to.

Remark 16: Note that as every element of $\mathcal{T}^{(n)}$ is nonnegative, it follows that every event reset state is a normalized population vector (Definition 10).
Lemma 10: For an independent Markovian sequence,

$$
\mathcal{T}^{(n-1)} V^{(n-1)}(t)=\tau^{(n)}(t) V_{R}^{(n)}
$$

Proof. The proof follows from the definition of the event reset state, Definition 15 and the event delay function, Definition 13, Eq. (D21).

Definition 16: A canonical independent Markovian sequence of events is an independent sequence (Definition 14) in which the initial state and every reset state (Definition 15) in the sequence is a canonical state (Definition 9). Alternatively, it is a sequence in which the initial state is a canonical state, and every event generator $\mathcal{T}^{(n)}$ has a single nonzero row.

Definition 17: For an independent sequence of Markovian events (Definition 14), one defines the subevent delay function as

$$
v^{(n)}(t)=\left\|\mathcal{T}^{(n-1)} e^{\mathcal{N}^{(n-1)} t} V_{R}^{(n-1)}\right\|_{\Sigma} .
$$

Remark 17: The $n$th subevent delay function refers to the delay function of the $n$th event from the time of occurrence of event $n-1$, as opposed to the $n$th delay function, which is constructed with respect to the initial time $t=0$.

Remark 18: That $v^{(n)}$ is indeed a delay function follows from Corollary 6. Note that $v^{(n)}(t)$ is independent of all other quantities in the sequence other than those that determine the occurrence of the $n$th event, namely the stochastic generators $\left\{\mathcal{N}^{(n-1)}, \mathcal{T}^{(n-1)}\right\}$ and the event reset state $V_{R}^{(n-1)}$.

Lemma 11: (The states and delay functions of an independent Markovian sequence.) For an independent Markovian sequence of events (Definition 14), every vector and delay function in the sequence can be expressed recursively for $n \geq 1$ as

$$
\begin{aligned}
V^{(n)}(t) & =\int_{0}^{t} e^{\mathcal{N}^{(n)}\left(t-t^{\prime}\right)} V_{R}^{(n)} \tau^{(n)}\left(t^{\prime}\right) d t^{\prime}, \\
\tau^{(n)}(t) & =\left(\tau^{(n-1)} * v^{(n)}\right)(t),
\end{aligned}
$$

where $v^{(n)}(t)$ is the $n$th subevent delay function, Definition 17. Note that $\tau^{(0)}(t)=\delta(t)$ by definition (Definition 13), and we equate the reset state for the zeroth event $V_{R}^{(0)}$ to the initial state $V^{(0)}(0)$.

It follows that the delay function of the $n$th event is the sequential convolution of the delay function of all previous 
events,

$$
\tau^{(n)}(t)=\left(v^{(1)} * v^{(2)} * \cdots * v^{(n)}\right)(t),
$$

and the state of the $n$th event is similarly given by the sequential convolution

$$
V^{(n)}(t)=\int_{0}^{t} e^{\mathcal{N}^{(n)}\left(t-t^{\prime}\right)} V_{R}^{(n)}\left(v^{(1)} * v^{(2)} * \cdots * v^{(n)}\right)\left(t^{\prime}\right) d t^{\prime} .
$$

Proof. The first statement of the lemma, Eq. (D44), follows from Lemmas 7 and 10. For the second statement [Eq. (D45)], we take the definition of the delay function [Eq. (D21)]. For $n \geq 1$,

$$
\begin{aligned}
\tau^{(n)}(t) & =\left\|\mathcal{T}^{(n-1)} \int_{0}^{t} e^{\mathcal{N}^{(n-1)}\left(t-t^{\prime}\right)} V_{R}^{(n-1)} \tau^{(n-1)}\left(t^{\prime}\right) d t^{\prime}\right\|_{\Sigma} \\
& =\int_{0}^{t}\left\|\mathcal{T}^{(n-1)} e^{\mathcal{N}^{(n-1)}\left(t-t^{\prime}\right)} V_{R}^{(n-1)}\right\|_{\Sigma} \tau^{(n-1)}\left(t^{\prime}\right) d t^{\prime} \\
& =\int_{0}^{t} v^{(n)}\left(t-t^{\prime}\right) \tau^{(n-1)}\left(t^{\prime}\right) d t^{\prime} \\
& =\left(\tau^{(n-1)} * v^{(n)}\right)(t),
\end{aligned}
$$

where $v^{(n)}$ is the subevent delay function, Definition 17. Proceeding by induction, one recovers Eqs. (D46) and (D47).

Lemma 12: In an independent Markovian sequence of events (Definition 14), if a pair of stochastic generators is scaled as

$$
\left\{\mathcal{N}^{(n)}, \mathcal{T}^{(n)}\right\} \rightarrow\left\{a \mathcal{N}^{(n)}, a \mathcal{T}^{(n)}\right\}
$$

where $a>0$, the delay function of the $m$ th event in the sequence, where $m>n$, is modified to be

$$
\begin{gathered}
\tau^{(m)}(t)=\left(v^{(1)} * v^{(2)} * \cdots * \tilde{v}^{(n+1)} *\right. \\
\\
\left.\cdots * v^{(m)}\right)(t), \\
\text { where } \tilde{v}^{(n+1)}(t)=a \cdot v^{(n+1)}(a t) .
\end{gathered}
$$

For $m \leq n$, the delay function is left unchanged.

Proof. The sequence is still independent, as scaling up the event generator $\mathcal{T}^{(n)}$ by a positive constant does not change its rank. Thus Lemma 11 still applies, and by Eq. (D46), one only has to consider the modification to the $(n+1)$ th subevent delay function (see Definition 17),

$$
\begin{aligned}
\tilde{\nu}^{(n+1)} & =\left\|a \cdot \mathcal{T}^{(n-1)} e^{a \cdot \mathcal{N}^{(n-1)} t} V_{R}^{(n-1)}\right\|_{\Sigma} \\
& =a\left\|\mathcal{T}^{(n-1)} e^{\mathcal{N}^{(n-1)}(a t)} V_{R}^{(n-1)}\right\|_{\Sigma} \\
& =a \cdot v^{(n+1)}(a t) .
\end{aligned}
$$

Lemma 13: (The delay function of a sequence in terms of canonical independent sequences.) Given a Markovian sequence (Definition 13), the $n$th delay function and state (for $n \geq 1$ ) in the sequence may be expressed in terms of convolutions of subevent delay functions (Definition 17) as follows:

$$
\begin{aligned}
\tau^{(n)}(t)= & \sum_{i_{0}=1}^{d^{(0)}} \sum_{i_{1}=1}^{d^{(1)}} \cdots \sum_{i_{n}=1}^{d^{(n)}} v_{i_{0}}\left(v_{i_{0} i_{1}}^{(1)} * v_{i_{1} i_{2}}^{(2)} * \cdots * v_{i_{n-1} i_{n}}^{(1)}\right)(t), \\
V^{(n)}(t)= & \int_{0}^{t} e^{\mathcal{N}^{(n)}\left(t-t^{\prime}\right)} \sum_{i_{0}=1}^{d^{(0)}} \sum_{i_{1}=1}^{d^{(1)}} \cdots \sum_{i_{n}=1}^{d^{(n)}} \mathbf{e}_{i_{n}}^{(n)} v_{i_{0}} \\
& \times\left(v_{i_{0} i_{1}}^{(1)} * v_{i_{1} i_{2}}^{(2)} * \cdots * v_{i_{n-1} i_{n}}^{(1)}\right)\left(t^{\prime}\right) d t^{\prime},
\end{aligned}
$$

where $v_{i_{0}}$ are the coefficients of the initial state in its canonical basis,

$$
V^{(0)}(0)=\sum_{i_{0}}^{d^{(0)}} v_{i_{0}} \mathbf{e}_{i_{0}}^{(0)},
$$

the canonical subevent delay functions $v_{i_{j-1} i_{j}}$ are defined as

$$
\begin{aligned}
v_{i_{j-1} i_{j}}^{j}(t) & =\left\|\mathcal{T}_{i_{j}}^{(j-1)} e^{\mathcal{N}^{(j-1)} t} \mathbf{e}_{i_{j-1}}^{(j-1)}\right\|_{\Sigma}, \\
i_{j-1} & \in\left\{1,2, \ldots, d^{(j-1)}\right\}, i_{j} \in\left\{1,2, \ldots, d^{(j)}\right\},
\end{aligned}
$$

and in turn the canonical event generators $\mathcal{T}_{i_{j}}^{(j-1)}$ are defined to be the original generator $\mathcal{T}^{(j-1)}$ with all the rows except row $i_{j}$ set to zero. Thus

$$
\begin{aligned}
\mathcal{T}^{(j-1)} & =\sum_{i_{j}=1}^{d^{(j)}} \mathcal{T}_{i_{j}}^{(j-1)}, \text { where } \\
{\left[\mathcal{T}_{i_{j}}^{(j-1)}\right]_{k l} } & =\delta_{i_{j}, k}\left[\mathcal{T}^{(j-1)}\right]_{k l} .
\end{aligned}
$$




\section{Remark 19: Explanation of Lemma 13.}

The difference between a canonical independent Markovian sequence (Definition 16) and a general Markovian sequence (Definition 13) is as follows. In the first case, the initial state and the states following the occurrence of every event in the sequence are canonical states, which is equivalent to every event generator in the sequence having a single nonzero row. For a general sequence, this is not the case.

However, even in the case of a general sequence, one can split each event generator into the sum of matrices with single nonzero rows, each corresponding to a single canonical state (hence the term canonical generators). Note from Lemma 7 that the states and delay functions of every event are linear with respect to the event generators, and thus by splitting the event generators of a general sequence into canonical generators, we find that states and delay functions may be expressed as a linear combination of each of the terms in the split. Thus the general sequence turns into a treelike graph, where each event corresponds to branching out with respect to all the possible canonical states after the event has occurred.

In other words, we decompose a Markovian sequence into the sum of all the possible canonical independent Markovian sequences that occur within it, and the above lemma expresses the states and delay functions of the original sequence as the same sum over the canonical sequences.

Proof. Proof by induction. Consider the case of $n=1$. For the delay function $\tau^{(1)}$, from Eq. (D21),

$$
\tau^{(1)}=\left\|\mathcal{T}^{(0)} V^{(0)}(t)\right\|_{\Sigma} .
$$

We may use the solution for $V^{(0)}(t)$ from Lemma 7, and the decomposition of the initial state in the canonical basis [Eq. (D60)],

$$
V^{(0)}(t)=e^{\mathcal{N}^{(0)} t} V^{(0)}(0)=\sum_{i_{0}=1}^{d^{(0)}} e^{\mathcal{N}^{(0)} t} v_{i_{0}} \mathbf{e}_{i_{0}}^{(0)} .
$$

We also split the event generator $\mathcal{T}^{(0)}$ into the sum of canonical generators [Eq. (D62)],

$$
\mathcal{T}^{(0)}=\sum_{i_{1}=1}^{d^{(1)}} \mathcal{T}_{i_{1}}^{(0)}
$$

Substituting these back into the delay function of the first event [Eq. (D63)], one obtains

$$
\tau^{(1)}=\sum_{i_{0}=1}^{d^{(0)}} \sum_{i_{1}=1}^{d^{(1)}} v_{i_{0}}\left\|\mathcal{T}_{i_{1}}^{(0)} e^{\mathcal{N}^{(0)} t} \mathbf{e}_{i_{0}}^{(0)}\right\|_{\Sigma}
$$

$$
=\sum_{i_{0}=1}^{d^{(0)}} \sum_{i_{1}=1}^{d^{(1)}} v_{i_{0}} v_{i_{0} i_{1}}^{(1)}
$$

using the definition of the canonical subevent delay function [Eq. (D61)]. Thus $\tau^{(1)}$ satisfies the statement of the lemma.

For the state $V^{(1)}$, we apply Lemma 7 ,

$$
V^{(1)}(t)=\int_{0}^{t} e^{\mathcal{N}^{(1)}\left(t-t^{\prime}\right)} \mathcal{T}^{(0)} V^{(0)}\left(t^{\prime}\right) d t^{\prime},
$$

and follow the same procedure of decomposing the state and event generator, resulting in

$$
V^{(1)}(t)=\int_{0}^{t} e^{\mathcal{N}^{(1)}\left(t-t^{\prime}\right)} \sum_{i_{0}=1}^{d^{(0)}} \sum_{i_{1}=1}^{d^{(1)}} v_{i_{0}}\left(\mathcal{T}_{i_{1}}^{(0)} e^{\mathcal{N}^{(0)} t^{\prime}} \mathbf{e}_{i_{0}}^{(0)}\right) d t^{\prime}
$$

The term in parentheses above is proportional to $\mathbf{e}_{i_{1}}^{(1)}$, as the generator $\mathcal{T}_{i_{1}}^{(0)}$ has only a single nonzero row, which is row $i_{1}$, corresponding to the canonical state $\mathbf{e}_{i_{1}}^{(1)}$. Furthermore, the e sum of the term has already been defined as the canonical subevent delay function [Eq. (D61)], and thus we can express it as

$$
\mathcal{T}_{i_{1}}^{(0)} e^{\mathcal{N}^{(0)} t^{\prime}} \mathbf{e}_{i_{0}}^{(0)}=\mathbf{e}_{i_{1}}^{(1)} v_{i_{0} i_{1}}^{(1)}\left(t^{\prime}\right)
$$

Substituting this back into Eq. (D69),

$$
V^{(1)}(t)=\int_{0}^{t} e^{\mathcal{N}^{(1)}\left(t-t^{\prime}\right)} \sum_{i_{0}=1}^{d^{(0)}} \sum_{i_{1}=1}^{d^{(1)}} v_{i_{0}} \mathbf{e}_{i_{1}}^{(1)} v_{i_{0} i_{1}}^{(1)}\left(t^{\prime}\right) d t^{\prime},
$$

which satisfies the statement of the lemma.

Proceeding, assume that the lemma is satisfied up to and including $n=k$. For the next delay function $\tau^{(k+1)}$, from Eq. (D21), 


$$
\begin{aligned}
\tau^{(k+1)} & =\left\|\mathcal{T}^{(k)} V^{(k)}(t)\right\|_{\Sigma} \\
& =\left\|\sum_{i_{k+1}=1}^{d^{(k+1)}} \mathcal{T}_{i_{k+1}}^{(k)} \int_{0}^{t} e^{\mathcal{N}^{(k)}\left(t-t^{\prime}\right)} \sum_{i_{0}=1}^{d^{(0)}} \sum_{i_{1}=1}^{d^{(1)}} \cdots \sum_{i_{k}=1}^{d^{(k)}} \mathbf{e}_{i_{k}}^{(k)} v_{i_{0}}\left(v_{i_{0} i_{1}}^{(1)} * v_{i_{1} i_{2}}^{(2)} * \cdots * v_{i_{k-1} i_{k}}^{(k)}\right)\left(t^{\prime}\right) d t^{\prime}\right\|_{\Sigma} \\
& =\sum_{i_{0}=1}^{d^{(0)}} \sum_{i_{1}=1}^{d^{(1)}} \ldots \sum_{i_{k+1}=1}^{d^{(k+1)}} v_{i_{0}} \int_{0}^{t}\left\|\mathcal{T}_{i_{k+1}^{(k)}} e^{\mathcal{N}^{(k)}\left(t-t^{\prime}\right)} \mathbf{e}_{i_{k}}^{(k)}\right\|_{\Sigma}\left(v_{i_{0} i_{1}}^{(1)} * v_{i_{1} i_{2}}^{(2)} * \cdots * v_{i_{k-1} i_{k}}^{(k)}\right)\left(t^{\prime}\right) d t^{\prime},
\end{aligned}
$$

where we apply the lemma for the state $V^{(k)}(t)$, and then simply rearrange terms (all of the states, sums are finite, and so is the integral).

Identifying the term in the e sum as another canonical subevent delay function [Eq. (D61)], we get

$$
\begin{aligned}
\tau^{(k+1)} & =\sum_{i_{0}=1}^{d^{(0)}} \sum_{i_{1}=1}^{d^{(1)}} \cdots \sum_{i_{k+1}=1}^{d^{(k+1)}} v_{i_{0}} \int_{0}^{t} v_{i_{k} i_{k+1}}^{(k+1)}\left(t-t^{\prime}\right)\left(v_{i_{0} i_{1}}^{(1)} * v_{i_{1} i_{2}}^{(2)} * \cdots * v_{i_{k-1} i_{k}}^{(k)}\right)\left(t^{\prime}\right) d t^{\prime} \\
& =\sum_{i_{0}=1}^{d^{(0)}} \sum_{i_{1}=1}^{d^{(1)}} \cdots \sum_{i_{k+1}=1}^{d^{(k+1)}} v_{i_{0}}\left(v_{i_{0} i_{1}}^{(1)} * v_{i_{1} i_{2}}^{(2)} * \cdots * v_{i_{k-1} i_{n}}^{(k)} * v_{i_{k} i_{k+1}}^{(k+1)}\right)(t)
\end{aligned}
$$

Thus $\tau^{(k+1)}$ also satisfies the lemma.

Finally, we express $V^{(k+1)}$ using Lemma 7,

$$
\begin{aligned}
V^{(k+1)}(t) & =\int_{0}^{t} e^{\mathcal{N}^{(k+1)}\left(t-t^{\prime}\right)} \mathcal{T}^{(k)} V^{(k)}\left(t^{\prime}\right) d t^{\prime} \\
& =\int_{0}^{t} e^{\mathcal{N}^{(k+1)}\left(t-t^{\prime}\right)} \mathcal{T}^{(k)} \int_{0}^{t^{\prime}} e^{\mathcal{N}^{(k)}\left(t^{\prime}-t^{\prime \prime}\right)} \sum_{i_{0}=1}^{d^{(0)}} \sum_{i_{1}=1}^{d^{(1)}} \cdots \sum_{i_{k}=1}^{d^{(k)}} \mathbf{e}_{i_{k}}^{(k)} v_{i_{0}}\left(v_{i_{0} i_{1}}^{(1)} * v_{i_{1} i_{2}}^{(2)} * \cdots * v_{i_{k-1} i_{k}}^{(k)}\right)\left(t^{\prime \prime}\right) d t^{\prime \prime} d t^{\prime}
\end{aligned}
$$

where we apply the lemma to $V^{(k)}$. Once again, we can split the generator $\mathcal{T}^{(k)}$ into canonical generators [Eq. (D62)], and rearrange the integral and sums appropriately,

$$
V^{(k+1)}(t)=\int_{0}^{t} e^{\mathcal{N}^{(k+1)}\left(t-t^{\prime}\right)} \sum_{i_{0}=1}^{d^{(0)}} \sum_{i_{1}=1}^{d^{(1)}} \ldots \sum_{i_{k+1}=1}^{d^{(k+1)}} \int_{0}^{t^{\prime}} \mathcal{T}_{i_{k+1}}^{(k)} e^{\mathcal{N}^{(k)}\left(t^{\prime}-t^{\prime \prime}\right)} \mathbf{e}_{i_{k}}^{(k)} v_{i_{0}}\left(v_{i_{0} i_{1}}^{(1)} * v_{i_{1} i_{2}}^{(2)} * \cdots * v_{i_{k-1} i_{k}}^{(k)}\right)\left(t^{\prime \prime}\right) d t^{\prime \prime} d t^{\prime}
$$

Once again, we can associate one of the terms above to a canonical subevent delay function [Eq. (D61)], as we did in Eq. (D70),

$$
\mathcal{T}_{i_{k+1}}^{(k)} e^{\mathcal{N}^{(k)}\left(t^{\prime}-t^{\prime \prime}\right)} \mathbf{e}_{i_{k}}^{(k)}=\mathbf{e}_{i_{k+1}}^{(k+1)} v_{i_{k} i_{k+1}}^{(k+1)}\left(t^{\prime}-t^{\prime \prime}\right),
$$

which results in

$$
\begin{aligned}
V^{(k+1)}(t) & =\int_{0}^{t} e^{\mathcal{N}^{(k+1)}\left(t-t^{\prime}\right)} \sum_{i_{0}=1}^{d^{(0)}} \sum_{i_{1}=1}^{d^{(1)}} \cdots \sum_{i_{k+1}=1}^{d^{(k+1)}} \int_{0}^{t^{\prime}} \mathbf{e}_{i_{k+1}}^{(k+1)} v_{i_{k} i_{k+1}}^{(k+1)}\left(t^{\prime}-t^{\prime \prime}\right) v_{i_{0}}\left(v_{i_{0} i_{1}}^{(1)} * v_{i_{1} i_{2}}^{(2)} * \cdots * v_{i_{k-1} i_{k}}^{(1)}\right)\left(t^{\prime \prime}\right) d t^{\prime \prime} d t^{\prime} \\
& =\int_{0}^{t} e^{\mathcal{N}^{(k+1)}\left(t-t^{\prime}\right)} \sum_{i_{0}=1}^{d^{(0)}} \sum_{i_{1}=1}^{d^{(1)}} \cdots \sum_{i_{k+1}=1}^{d^{(k+1)}} \mathbf{e}_{i_{k+1}}^{(k+1)} v_{i_{0}}\left(v_{i_{0} i_{1}}^{(1)} * v_{i_{1} i_{2}}^{(2)} * \cdots * v_{i_{k-1} i_{k}}^{(k)} * v_{i_{k} i_{k+1}}^{(k+1)}\right)\left(t^{\prime}\right) d t^{\prime}
\end{aligned}
$$

which also satisfies the lemma. By induction, the lemma applies for all $n \geq 1$. 


\section{Result on the accuracy of Markovian sequences}

Theorem 3: Given a Markovian sequence (Definition 13), there exists a canonical independent Markovian sequence (Definition 16) such that

(a) the vectors and generators of the canonical sequence are of the same dimension as the original sequence, i.e., $d^{(n)}$ remain the same for all $n$, and

(b) the accuracies (Definition 7) of every event in the canonical sequence, i.e., the accuracies of each of the event delay functions [Eq. (D21)] upper bounds those of the original sequence.

Furthermore, one such canonical independent Markovian sequence can be explicitly constructed from the original sequence by

(a) changing the initial state to a well chosen canonical state,

(b) making all of the event generators $\mathcal{T}^{(n)}$ into rank1 matrices by keeping only a single nonzero row, possibly shifted to a different row, and

(c) scaling each pair of event generators $\left\{\mathcal{N}^{(n)}, \mathcal{T}^{(n)}\right\}$ by well-chosen positive constants.

Remark 20: The major implication of this theorem is that if one's interest is in upper bounding the accuracy of Markovian sequences, then it suffices to optimize over the much smaller subset of canonical independent Markovian sequences, which are far more tractable.

Our main use for this theorem is to straightforwardly apply it to imply that "reset" clocks, i.e., those that go to a fixed state every time they tick, are the most accurate. However, the content of this theorem is more general than its application to clocks.

Proof. We begin by restating the first part of Lemma 13, which states that each of the delay functions of a Markovian sequence of events may be expressed as

$$
\tau^{(n)}(t)=\sum_{i_{0}=1}^{d^{(0)}} \sum_{i_{1}=1}^{d^{(1)}} \cdots \sum_{i_{n}=1}^{d^{(n)}} v_{i_{0}}\left(v_{i_{0} i_{1}}^{(1)} * v_{i_{1} i_{2}}^{(2)} * \cdots * v_{i_{n-1} i_{n}}^{(1)}\right)(t)
$$

where the canonical subevent delay functions $v_{i_{j-1} i_{j}}$ are defined in Eq. (D61), with the associated canonical event generators subsequently in Eq. (D62).

Applying Lemma 4 regarding the accuracy of mixtures of delay functions to Eq. (D83),

$$
R\left[\tau^{(n)}\right] \leq \max _{i_{0}, i_{1}, \ldots, i_{n}} R\left[v_{i_{0} i_{1}}^{(1)} * v_{i_{1} i_{2}}^{(2)} * \cdots * v_{i_{n-1} i_{n}}^{(1)}\right],
$$

where the range of each $i_{j}$ above (and in what follows) is $\left\{1,2, \ldots, d^{(j)}\right\}$. We follow by applying Lemma 2 regarding the accuracy of convolutions of delay functions, and find that

$R\left[\tau^{(n)}\right] \leq \max _{i_{0}, i_{1}, \ldots, i_{n}}\left(R\left[v_{i_{0} i_{1}}^{(1)}\right]+R\left[v_{i_{1} i_{2}}^{(2)}\right]+\cdots+R\left[v_{i_{n-1} i_{n}}^{(n)}\right]\right)$.

In the maximization above, each $i_{j}$ for $1 \leq j \leq n-1$ appears twice, in $v_{i_{j-1} i_{j}}^{(j)}$ and $v_{i_{j} i_{j+1}}^{(j+1)}$. Relaxing the maximization to allow for independent maximization of each accuracy, we end up with

$$
\begin{aligned}
R\left[\tau^{(n)}\right] \leq & \max _{i_{0}, i_{1}^{\prime}, i_{1}, i_{2}^{\prime}, i_{2}, \ldots, i_{n-1}^{\prime}, i_{n-1}, i_{n}} \\
& \times\left(R\left[v_{i_{0} i_{1}^{\prime}}^{(1)}\right]+R\left[v_{i_{1} i_{2}^{\prime}}^{(2)}\right]+\cdots+R\left[v_{i_{n-2} i_{n-1}^{\prime}}^{(n-1)}\right]\right. \\
& \left.+R\left[v_{i_{n-1}}^{(n)}\right]\right) .
\end{aligned}
$$

In other words, if we label by $R_{j}^{\max }$ the best accuracy from among the subevent delay functions of the $j$ th event,

$$
R_{j}^{\max }=\max _{i_{j-1}, i_{j}} R\left[v_{i_{j-1} i_{j}}^{(j)}\right],
$$

then the accuracy of the $n$th event in the sequence is bounded by

$$
R\left[\tau^{(n)}\right] \leq \sum_{j=1}^{n} R_{j}^{\max }
$$

To complete the proof, we construct a canonical independent Markovian sequence from the original sequence, that saturates the above inequality.

Consider that one performs the maximization in Eq. (D87) for every event $j \in\{1,2, \ldots, n\}$. Doing so for the $j$ th event will return the optimal indices $i_{j-1}$ and $i_{j}$, that we label as $l_{j-1}$ and $m_{j}$, respectively. We also label the optimal canonical subevent delay function as $\zeta^{(j)}$,

$$
\zeta^{(j)}=v_{l_{j-1} m_{j}}^{(j)} .
$$

In other words, the optimal canonical subevent delay function for the $j$ th event is obtained by starting in the canonical state $\mathbf{e}_{l_{j-1}}^{(j-1)}$, and associated with the canonical event generator $\mathcal{T}_{m_{j}}^{(j)}\left[\right.$ Eq. (D62)] that results in the state $\mathbf{e}_{m_{j}}^{(j)}$ when the event occurs.

Our first step in the construction of the new independent sequence is to take the original Markovian sequence, and change all of the event generators $\mathcal{T}^{(j)}$ into the optimal generators $\mathcal{T}_{m_{j}}^{(j)}$, by setting all of the rows except row $m_{j}$ to zero. The new sequence is now a canonical independent Markovian sequence (Definition 14). 
However, this is not enough, as we note that $m_{j} \neq l_{j}$ in general, i.e., the optimal canonical generator for the $j$ th event may not correspond to the optimal initial canonical state for event $j+1$. However, by Lemma 9, a delay function is left unchanged under operations that leave the column sums of its event generator the same. Since $\mathcal{T}_{m_{j}}^{(j)}$ has a single nonzero row, we shift the row from the $m_{j}$ position to the $l_{j}$ position, to obtain a new canonical generator $\tilde{\mathcal{T}}^{(j)}$, that is both optimal with respect to its own event as well as the initial canonical state for the next event.

By construction, we now have a canonical independent Markovian sequence, whose individual subevent delay functions (Definition 17) are the ones with the optimal accuracy, i.e., the $\zeta^{(j)}$ from Eq. (D89). From Lemma 11, the delay function of the $n$th event in the new sequence is now

$$
\tilde{\tau}^{(n)}(t)=\left(\zeta^{(1)} * \zeta^{(2)} * \cdots * \zeta^{(n)}\right)(t) .
$$

Applying Lemma 2 to the above delay function, we get that the accuracy is bounded by

$$
R\left(\tilde{\tau}^{(n)}\right) \leq \sum_{j=1}^{n} R\left(\zeta^{(j)}\right)=\sum_{j=1}^{n} R_{j}^{\max } .
$$

From Lemma 2, equality holds only if the mean $\mu$ of each optimal delay function $\zeta^{(j)}$ satisfies

$$
\frac{\mu\left(\zeta^{(j)}\right)}{R\left(\zeta^{(j)}\right)}=\frac{\mu\left(\zeta^{(j)}\right)}{R\left(\zeta^{(j)}\right)} \quad \forall j, k \in\{1,2, \ldots, n\}
$$

This is not automatically true from our construction so far. However, from Lemma 12, we can scale the $j$ th pair of event generators by a positive constant $a_{j}$ in the sequence to change the subevent delay function from $\zeta^{(j)}(t)$ to $a \zeta^{(j)}(a t)$, leaving every other subevent delay function unchanged. Furthermore, by Lemma 5, such a scaling operation does not affect the accuracy of the subevent delay function, but does scale its mean (the new mean is divided by $a_{j}$ ).

Thus we can pick a series of positive constants $\left\{a_{j}\right\}$ to scale each pair of generators in the independent sequence we construct so that every subevent delay function has the same ratio of mean to accuracy, and thus satisfies Eq. (D92).

As a result, we are left with the canonical independent sequence satisfying the statement of the lemma, whose accuracy saturates Eq. (D88), which itself is an upper bound to the accuracy of the original sequence.

\section{APPENDIX E: CLASSICAL CLOCKS}

In this part of the Appendix, we review the behavior of classical clocks, showing that reset clocks (those with a fixed state after ticking) can reach the highest accuracy, and that, for a classical clock of dimension $d$, the accuracy is upper bound by its dimension. Finally, we discuss a simple classical clock that saturates this bound.

In fact, the dynamics of classical clocks, at least those that are self-contained, is a subset of more general Markovian dynamics, described by the Kolmogorov equation [53]. The ticks of a finite-dimensional clock may be understood as a sequence of events generated by Markovian dynamics on a finite-dimensional vector space. As such, we include Appendix D that introduces the mathematics of Markovian sequences that we require to prove our main results on clocks. Furthermore, we draw on some lemmas on the behavior of the accuracy of mixtures and sequences of delay functions, which are covered in Appendix 1 (where delay functions and the accuracy $R$ are discussed).

Appendices B 1 and D 1 derive from standard theory on stochastic process and random variables, and are included here to place clocks within the larger context of the theory of stochastic processes, and for ease of reading and understanding of the proofs of our main results, which rely on multiple lemmas within this theory. For an indepth discussion on stochastic processes, see, for example, Ref. [51].

In Appendix E 1, we introduce classical clocks and they were discussed in the main text, and relate them to Markovian sequences. Reset clocks, those that tick to a fixed state, are discussed and shown to be the same as independent Markovian sequences.

In Appendix E 2, we first apply Theorem 3 to clocks to obtain our first result on clocks, that for every classical clock, there is a reset clock of at least as high accuracy. Furthermore, in Appendix E 2 a, by focusing on the accuracy of reset clocks, we find that the quantity $R$ that we denote as the accuracy is in fact a good quantifier of how long the clock can run before being in error.

Our final result on classical clocks, that their accuracy $R$ is upper bound by their dimension, is stated and proven in Appendix E 3.

\section{Classical clocks}

Definition 18: As discussed in the main text (Sec. II D, a self-contained stochastic finite-dimensional classical clock, or for simplicity, a classical clock, is represented by a time-dependent vector $V(t) \in \mathbb{R}^{d}$, that represents the state of the clock, such that $V(0)$ is a population vector (Definition 10), together with a pair of time-independent stochastic generators $\{\mathcal{N}, \mathcal{T}\}$ (Definition 12), that generate the dynamics of the clock via the relation

$$
\frac{d}{d t} V(t)=(\mathcal{N}+\mathcal{T}) V(t)
$$

and where the probability per unit time of a tick being observed, that we label the "tick density" and denote by 
$p_{\text {tick }}(t)$, is given by

$$
p_{\text {tick }}(t)=\|\mathcal{T} V(t)\|_{\Sigma} .
$$

Remark 21: The clock is self-contained because its generators are time independent, and finite dimensional as the vector space of clock states is taken to be finite dimensional. Finally, the clock is stochastic (i.e. classical), as the states are population vectors evolving under stochastic generators, which is equivalent, in the context of quantum theory, to restricting the initial state to be diagonal in some preferred basis, and restricting the dynamical generators to Lindbladian operators that keep the states diagonal in the same basis, as discussed in Sec. II D of the main text.

In the entirety of this work, we consider only selfcontained and finite-dimensional clocks. For simplicity, we continue for the remainder of this Appendix by shortening the term to simply classical clocks, with the implicit understanding that they are also self-contained, and finite dimensional, and that the term classical implies stochastic.

Remark 22: In the context of classical clocks, since $\mathcal{T}$ is associated to the generation of ticks, we refer to it as the tick generator, and to $\mathcal{N}$ as the nontick generator.

\section{a. Tick states and tick delay functions}

The state $V(t)$ and tick density $p_{\text {tick }}(t)$ in the above description of classical clocks (Definition 18) do not contain any information about how many times the clock has already ticked in the past. In order to distinguish between each tick, one can split the state into a sequence $\left\{V^{(n)}\right\}$ of "tick states," each corresponding to a fixed number of ticks.

Definition 19: The tick states of a classical clock (Definition 18) are a sequence of states $\left\{V^{(n)}(t)\right\}, n \in$ $\{0,1,2, \ldots\}$, where the initial conditions and dynamics for each state in the sequence are determined with respect to the pair of stochastic generators $\{\mathcal{N}, \mathcal{T}\}$ of the clock as

$$
\begin{aligned}
V^{(n)}(0) & = \begin{cases}V(0) & \text { for } n=0, \\
0 & \text { for } n>0,\end{cases} \\
\text { and } \frac{d}{d t} V^{(n)}(t) & = \begin{cases}\mathcal{N} V_{0}(t) & \text { for } n=0, \\
\mathcal{N} V^{(n)}(t)+\mathcal{T} V^{(n-1)}(t) & \text { for } n>0 .\end{cases}
\end{aligned}
$$

Remark 23: The choice of initial conditions [Eq. (E3a)] corresponds to the fact that the clock has not ticked yet, and thus the entire state of the clock is associated with $V^{(0)}$, the state corresponding to no ticks.

For $t>0$, in every infinitesimal time interval, the tick generator moves probability from a state in the sequence to the next, while the nontick generator keeps the probability within the same tick subspace (albeit moving it around, and possibly decreasing it). Thus each $V^{(n)}(t)$ evolves due to only two contributions: firstly, its own evolution, as $\mathcal{N} V^{(n)}(t)$, and because of a tick, from $\mathcal{T} V^{(n-1)}(t)$.

In an analogous manner to the state, one can split the tick density [Eq. (E2)] of the clock into the contributions of the first, second, and following ticks.

Definition 20: We define the tick delay function of the $n$th tick to be

$$
\tau^{(n)}(t)=\left\|\mathcal{T} V^{(n-1)}(t)\right\|_{\Sigma} .
$$

Remark 24: The expression for the $n$th tick delay function reflects the fact that the $n$th tick can only occur after $n-1$ ticks, and the corresponding state of the clock is $V^{(n-1)}(t)$.

Remark 25: By construction, the tick states (Definition 19) and the tick delay functions (Definition 20) of a classical clock form a Markovian sequence of events (Definition 13). In the case of clocks, however, the dynamics of every event (tick) is identical to the previous one, featuring the same pair of generators on the same vector space.

\section{b. Reset clocks}

In our discussion on Markovian sequences of events, it was useful to discuss independent Markovian sequences (Definition 14), those that had fixed states following the occurrence of each event. The equivalent for clocks is a "reset clock," which we now characterize.

Definition 21: A classical reset clock (in this Appendix simply a reset clock) is a classical clock (Definition 18) for which the event of ticking always causes the clock to return to a fixed state, which is also the initial state, i.e.,

$$
\mathcal{T} V \propto V(0) \quad \forall V \in \mathbb{R}^{d} .
$$

Equivalently, a reset clock is one for which the tick generator $\mathcal{T}$ is a rank-1 operator, all of whose columns are proportional to the initial state $V(0)$.

Remark 26: By definition, the tick states and tick delay functions of a reset clock form an independent Markovian sequence of events, Definition 14. Since the events in the case of clocks are ticks, and the dynamics of each tick is generated identically to all the others, the ticks of a reset clock correspond to an IID sequence, i.e., a sequence of independent and identically distributed events [51].

\section{The accuracy of reset clocks}

\section{a. The accuracy $R$ quantifies the average run time of reset clocks}

If we apply Lemma 11 to the case of reset clocks (Definition 21), we find that the delay function of the $n$th 
tick of the clock is given by the $n$-fold convolution of the delay function of the first tick,

$$
\tau^{(n)}(t)=\left(\tau_{1} * \tau_{1} * \cdots * \tau_{1}\right)(t)
$$

If we denote the zeroth moment, first moment, second moment, variance and accuracy (Definitions 6 and 7) of a single tick of a reset clock (i.e., the delay function of a single tick) by $Q, \mu, \chi, \sigma, R$, respectively, and those of the $n$th tick by $Q^{(n)}, \mu^{(n)}, \chi^{(n)}, \sigma^{(n)}, R^{(n)}$, we find from the application of Eq. (B8) for the moments of a convolution of delay functions, and Eq. (B6) for the accuracy of a delay function, that

$$
\left\{\begin{array}{l}
Q^{(n)} \\
\mu^{(n)} \\
\chi^{(n)} \\
\sigma^{(n)} \\
R^{(n)}
\end{array}\right\}=\left\{\begin{array}{c}
Q^{n} \\
n \mu \\
n \chi+n(n-1) \mu^{2} \\
\sqrt{n} \cdot \sigma \\
n R
\end{array}\right\} .
$$

Consider that one asks the question "How many ticks can the clock produce until the uncertainty in the time of occurrence of the next tick has grown to be equal to the time interval between ticks?," which is a universally accepted mark of a clock's accuracy.

For reset clocks, the average time interval between ticks is $\mu^{(n)}-\mu^{(n-1)}=\mu$, and is independent of which tick we are at. On the other hand, the uncertainty in the time of occurrence of the $n$th tick, $\sigma^{(n)}=\sqrt{n} \sigma$, grows with the number of ticks. If we denote the average number of ticks before the uncertainty equals the interval between ticks as $N$,

$$
\begin{aligned}
\sigma^{(n)} & =\mu^{(n)}-\mu^{(n-1)}, \\
\therefore \sqrt{N} \sigma & =\mu, \\
\therefore N & =\frac{\mu^{2}}{\sigma^{2}}=R .
\end{aligned}
$$

\section{b. Result: The accuracy of all classical clocks is bound by that of reset classical clocks}

Theorem 4: For every classical clock (Definition 18), there exists a reset clock (Definition 21) of the same dimension such that the accuracies of the delay functions of every tick of the original clock are upper bounded by the corresponding accuracies of the reset clock. Furthermore, such a reset clock can be obtained from the original clock by

(a) picking a single well chosen canonical state to be the initial state,

(b) setting all but one of the rows of the tick generator $\mathcal{T}$ to be zero, and shifting the single nonzero row to the location corresponding to the initial canonical state.
Proof. The theorem follows from the direct application of Theorem 3 to the case of classical clocks, when one identifies the tick states (Definition 19) and tick delay functions (Definition 20) as a Markovian sequence of events (Definition 13), while those of a reset clock (Definition 21) are an independent Markovian sequence (Definition 14).

\section{An upper bound on the accuracy of classical clocks}

Theorem 5: For a classical clock of dimension $d$ (Definition 18), where $d \in \mathbb{N}^{+}$, the accuracy of its $n$th tick, i.e., the accuracy $R$ (Definition 7) of the delay function of the $n$th tick (Definition 20), is upper bound by

$$
R\left[\tau^{(n)}\right] \leq n d .
$$

In particular, for reset clocks of dimension $d$, which upper bound the accuracies of the ticks of arbitrary $d$ dimensional clocks (see Theorem 4), the accuracy of every single tick with respect to the previous one is upper bound by

$$
R[\tau] \leq d,
$$

which, as discussed in Sec. a, is a quantifier for the number of ticks outputted before the clock is expected to fail.

Proof. The rest of this section is dedicated to the proof of Theorem 5 . We prove the theorem by fixing the dimension $d$ and optimizing the accuracy over classical clocks of that dimension. From Theorem 4, we know that the accuracies of the ticks of any clock are upper bound by that of at least one reset clock with a canonical initial (and reset) state, and thus we may restrict our optimization to the case of reset clocks of dimension $d$ whose initial and reset state is the same canonical state.

Furthermore, as discussed in Sec. E 2 a, the accuracy of the $n$th tick of a reset clock is simply $n$ times the accuracy of the first tick, and the delay functions of every tick with respect to the time of occurrence of the previous tick are identical to each other, and equal to the delay function of the first tick.

Thus the quantity we are left to optimize is simply the accuracy of the first tick, and the restricted set we optimize over is that of reset clocks with a canonical initial state, and a canonical tick generator chosen so that the reset state is the initial state.

More precisely, we work in a $d$-dimensional real vector space, spanned by the canonical basis $\mathbf{e}_{i}$, where $i \in$ $\{0,1, \ldots, d-1\}$. Since the initial and reset state must be a canonical state, we label this as $\mathbf{e}_{0}$ without loss of generality. Thus the tick generator $\mathcal{T}$ has only one nonzero row, i.e., its first row, that corresponds to $\mathbf{e}_{0}$. On the other hand, 
the nontick generator $\mathcal{N}$ is arbitrary. The state and corresponding delay function of a single tick of the clock are thus

$$
\begin{aligned}
V(t) & =e^{\mathcal{N} t} \mathbf{e}_{0}, \\
\tau(t) & =\left\|\mathcal{T} e^{\mathcal{N} t} \mathbf{e}_{0}\right\|_{\Sigma} .
\end{aligned}
$$

To prove that the accuracy of the above delay function is bounded by the dimension $d$ for all $d \in \mathbb{N}^{+}$, we employ the method of induction, starting with the case $d=1$. In this case, both $\mathcal{N}$ and $\mathcal{T}$ are real numbers, that we label $\mathcal{N}=-p$ and $\mathcal{T}=g$, respectively, where $p \geq g>0$ in order to ensure that $\{\mathcal{N}, \mathcal{T}\}$ are a pair of stochastic generators (Definition 12). The delay function is thus

$$
\tau(t)=g e^{-p t} .
$$

One can calculate all of the moments (Definition 6) explicitly,

$$
\begin{aligned}
& Q=\left\langle t^{0}\right\rangle=\frac{g}{p}, \\
& \mu=\frac{\left\langle t^{1}\right\rangle}{Q}=\frac{1}{p}, \\
& \chi=\frac{\left\langle t^{2}\right\rangle}{Q}=\frac{2}{p^{2}},
\end{aligned}
$$

from which the $\gamma$ value [Eq. (B6)] is $\gamma=\chi / \mu^{2}=2$, and the accuracy is

$$
R[\tau]=\frac{1}{\gamma-1}=1,
$$

which satisfies the statement of the theorem.

We continue by assuming the theorem applies for dimensions $\{1,2, \ldots, d-1\}$, and prove that it applies to a $d$-dimensional clock.

The rest of the proof is structured as follows. In Sec. E 3 a, we divide the $d$-dimensional vector space of the clock into two subspaces, a one-dimensional space corresponding to the initial (canonical) state, and the complementary $d$-1-dimensional space, and define sequences of states and delay functions that correspond to this division. In Sec. E $3 \mathrm{~b}$, we prove that we can recover the dynamics of the state $V(t)$ and the delay function $\tau(t)$ of a single tick from these defined sequences. In Sec. E 3 c, we calculate the moments of the delay function $\tau(t)$ explicitly using the defined sequences, and upper bound the accuracy of $\tau(t)$.

\section{a. Dividing the vector space of the clock into a sum of one-dimensional and $\boldsymbol{d}-\mathbf{1}$-dimensional spaces}

In order to use the result for $d-1$-dimensional clocks, we divide the $d$-dimensional space of states into the direct sum of two subspaces, firstly, the one-dimensional space corresponding to the initial state $\mathbf{e}_{0}$, that we label $S_{0}$, and its complement, that is spanned by the rest of the canonical basis, that we label $S_{1}$. Thus $S_{0}$ is one dimensional, while $S_{1}$ is $d-1$ dimensional. We denote the projectors onto these spaces as $\Pi_{0}$ and $\Pi_{1}$. In matrix form with respect to the canonical basis, these projectors are

$$
\Pi_{0}=\left(\begin{array}{c|c}
1 & \mathbf{0} \\
\hline \mathbf{0} & \mathbf{0}
\end{array}\right), \Pi_{1}=\left(\begin{array}{c|c}
0 & \mathbf{0} \\
\hline \mathbf{0} & \mathbb{1}_{d-1}
\end{array}\right),
$$

where $\mathbb{1}_{d-1}$ represents the identity operator on a $d-1$ dimensional real vector space. Note that $\Pi_{0}+\Pi_{1}=\mathbb{1}_{d}$, corresponding to $S_{0} \oplus S_{1}=\mathbb{R}^{d}$.

One may split the nontick generator $\mathcal{N}$ into the corresponding sum of four matrices, with respect to the subspaces $S_{0}$ and $S_{1}$,

$$
\begin{aligned}
\mathcal{N} & =\left(\Pi_{0}+\Pi_{1}\right) \mathcal{N}\left(\Pi_{0}+\Pi_{1}\right) \\
& =\mathcal{N}_{00}+\mathcal{N}_{01}+\mathcal{N}_{10}+\mathcal{N}_{11},
\end{aligned}
$$

where $\mathcal{N}_{x y}=\Pi_{x} \mathcal{N} \Pi_{y}$.

Visually, this corresponds to the expressing $\mathcal{N}$ with respect to the canonical basis as

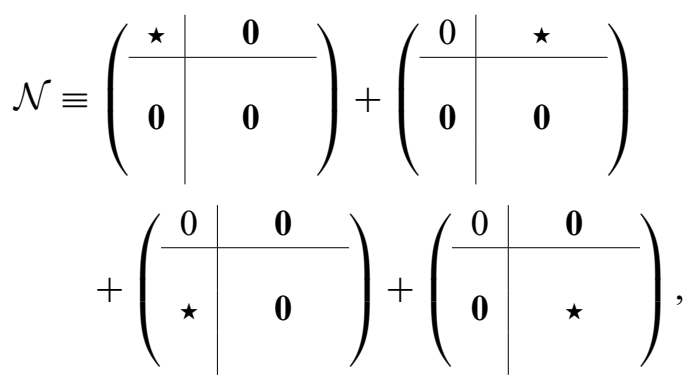

where $\star$ denote the original elements, and the matrices above are, respectively, $\mathcal{N}_{00}, \mathcal{N}_{01}, \mathcal{N}_{10} \mathcal{N}_{11}$.

One may understand each of the above generators $\mathcal{N}_{x y}$ as the part of the nontick generator that is responsible for moving the state from the subspace $S_{y}$ into the subspace $S_{x}$. More precisely, given the state of the clock at some time is $V(t)$, the part of the state that is in the subspace $S_{y}$ is given by the projection $\Pi_{y} V(t)$. On this part of the state, the infinitesimal change generated by the nontick generator is $\mathcal{N} \Pi_{y} V(t)$. Finally, the part of this infinitesimal state change that is in the space $S_{x}$ is the projection $\Pi_{x} \mathcal{N} \Pi_{y} V(t)$.

We follow the same procedure for the tick generator $\mathcal{T}$. However, $\mathcal{T}$ takes only states to the reset state $\mathbf{e}_{0}$, i.e., for 
all $V \in \mathbb{R}^{d}$,

$$
\mathcal{T} V \propto \mathbf{e}_{0} \quad \Longleftrightarrow \mathcal{T} V \in S_{0} \quad \Longleftrightarrow \quad \Pi_{1} \mathcal{T}=0,
$$

and thus

$$
\begin{aligned}
& \mathcal{T}=\mathcal{T}_{00}+\mathcal{T}_{01}, \\
& \text { where } \mathcal{T}_{x y}=\Pi_{x} \mathcal{T} \Pi_{y} \text {, } \\
& \text { and } \quad \mathcal{T}_{10}=\mathcal{T}_{11}=0 \text {. }
\end{aligned}
$$

At this point, we have split both of the generators into components that describe the movement of the state within and between the subspaces $S_{0}$ and $S_{1}$. We proceed to do the same for the state of the clock, and construct an independent Markovian sequence of states and corresponding delay functions that does this. Each state in the sequence must be distinguished by two indices, first, an $x \in\{0,1\}$ to denote that the state belongs to $S_{x}$, and $n \in \mathbb{N}^{0}=\{0,1,2, \ldots\}$ to mean that the state corresponds to population having moved from the initial state $\mathbf{e}_{0} \in S_{0}$ to the space $S_{1}$ and back $n$ times.

Definition 22: We define the set of path-specific clock states $v_{n, x}(t)$, where $n \in\{0,1,2, \ldots\}$ and $x \in\{0,1\}$, by the initial conditions

$$
v_{n, x}(0)= \begin{cases}\mathbf{e}_{0} & \text { if } n=x=0 \\ \mathbf{0} & \text { otherwise }\end{cases}
$$

and the dynamics

$$
\frac{d}{d t} v_{n, x}(t)= \begin{cases}\mathcal{N}_{00} v_{0,0}(t) & \text { if } n=x=0 \\ \mathcal{N}_{x x} v_{n, x}(t)+\mathcal{N}_{x \bar{x}} v_{n-\bar{x}, \bar{x}}(t), & \text { otherwise }\end{cases}
$$

where $\bar{x}=x \oplus 1$ is the complement of $x$.

Remark 27: Roughly speaking, $v_{n, x}(t)$ is the part of the state of the clock (of the first tick) corresponding to being in the subspace $S_{x}$ and having gone from $S_{0}$ to $S_{1}$ and back $n$ times.

Definition 23: We define the set of path-specific delay functions $\xi_{n, x}(t)$, where $n \in\{0,1,2, \ldots\}$ and $x \in\{0,1\}$, barring $n=x=0$, by

$$
\xi_{n, x}(t)=\left\|\mathcal{N}_{x \bar{x}} v_{n-\bar{x}, \bar{x}}(t)\right\|_{\Sigma} .
$$

Remark 28: $\xi_{n, x}(t)$ refers to the delay function of arriving at the $\{n, x\}$ state $v_{n, x}$ at time $t$.

In anticipation of dividing the delay function of a single tick of the clock using the above sequences, we define the following "component delay functions."
Definition 24: We define the set of component delay functions $\tau_{n, x}(t)$, where $n \in\{0,1,2, \ldots\}$ and $x \in\{0,1\}$, barring $n=x=0, b y$

$$
\tau_{n, x}(t)=\left\|\mathcal{T}_{0 \bar{x}} v_{n-\bar{x}, \bar{x}}(t)\right\|_{\Sigma} .
$$

Remark 29: The term "delay function" (Definition 5) for the above is appropriate. We shortly show that the $v_{n, x}(t)$ are an independent Markovian sequence of states, and the event generator $\mathcal{T}_{x \bar{x}}$, taken together with $\mathcal{N}_{\bar{x} \bar{x}}$, which is the relevant nonevent generator for the state $v_{n-\bar{x}, \bar{x}}(t)$, form a pair of stochastic generators $\left\{\mathcal{N}_{\bar{x} \bar{x}}, \mathcal{T}_{x \bar{x}}\right\}$ (Definition 12). From Corollary 6, we conclude that the $\tau_{n, x}(t)$ are delay functions.

Finally, as we eventually prove, the sequences above are found to involve repetitive convolutions of a small set of delay functions, which we proceed to define.

Definition 25: We define the pair of path-generating reset states $\mathbf{u}_{x}$, where $x \in\{0,1\}$ in the following manner. $\mathbf{u}_{0}=$ $\mathbf{e}_{0}$, and $\mathbf{u}_{1}$ is constructed by taking the single nonzero column of $\mathcal{N}_{10}$ [Eq. (E21)], and normalizing it.

Definition 26: We define the pair of path-generating delay functions $\Theta_{x}$, where $x \in\{0,1\}$ by

$$
\Theta_{x}=\left\|\mathcal{N}_{x \bar{x}} e^{\mathcal{N}_{\bar{x}} \bar{x}} \mathbf{u}_{\bar{x}}\right\|_{\Sigma},
$$

where $\mathbf{u}_{x}$, are the path-generating reset states (Definition 25).

Definition 27: We define the pair of tick-generating delay functions $\Gamma_{x}$, where $x \in\{0,1\}$ by

$$
\Gamma_{x}=\left\|\mathcal{T}_{0 \bar{x}} e^{\mathcal{N}_{\bar{x} \bar{x}} t} \mathbf{u}_{\bar{x}}\right\|_{\Sigma},
$$

where $\mathbf{u}_{x}$, are the path-generating reset states (Definition 25).

Remark 30: Put very simply, $\Theta_{x}$ is the delay function of the event of moving from $S_{\bar{x}}$ to $S_{x}$, while $\Gamma_{x}$ is the delay function of ticking from the subspace $S_{\bar{x}}$.

Remark 31: The above two definitions are justified in using the terminology "delay function" as both the pair $\left\{\mathcal{N}_{\bar{x} \bar{x}}, \mathcal{N}_{x \bar{x}}\right\}$ as well as the pair $\left\{\mathcal{N}_{\bar{x} \bar{x}}, \mathcal{T}_{x \bar{x}}\right\}$ can be proven to be pairs of stochastic generators, and from Corollary 6 , it follows that the $\Theta_{x}$ and $\Gamma_{x}$ defined above satisfy the requirements of a delay function (Definition 5).

\section{b. Proving the necessary properties of the path-specific states and delay functions.}

Lemma 14: The set of path-specific clock states (Definition 22) and delay functions (Definition 23) form an independent Markovian sequence of events (Definitions 13 and 14) with respect to the ordering where 
the event $\{n, x\}$ is followed by $\{n+x, \bar{x}\}$, corresponding to $\{\{0,0\},\{0,1\},\{1,0\},\{1,1\},\{2,0\},\{2,1\},\{3,0\}, \ldots\}$; and via the identification of $\left\{\mathcal{N}_{x x}, \mathcal{N}_{\bar{x} x}\right\}$ as the pair of event and nonevent stochastic generators for the event $\{n, x\}$.

Furthermore, the state $v_{n, x}(t) \in S_{x}$ for all $t \geq 0$, i.e.,

$$
\begin{aligned}
& \Pi_{0} v_{n, 0}(t)=v_{n, 0}(t) \quad \text { and } \quad \Pi_{1} v_{n, 1}(t)=v_{n, 1}(t) \quad \text { and } \\
& \Pi_{0} v_{n, 1}(t)=\Pi_{1} v_{n, 0}(t)=\mathbf{0} .
\end{aligned}
$$

Proof. Following the definition of a Markovian sequence of events, Definition 13, we note that the definition of the initial states in the sequence satisfy the definition by construction. In addition, we require the pairs of event generators for each event in the sequence, $\left\{\mathcal{N}_{x x}, \mathcal{N}_{\bar{x} x}\right\}$ to be a pair of stochastic generators, Definition 12.

First off, to prove that $\mathcal{N}_{x x}$ is a nonevent generator, note that since $\mathcal{N}_{x x}=\Pi_{x} \mathcal{N} \Pi_{x}$, each element of $\mathcal{N}_{x x}$ is equal to either the original value of the element in $\mathcal{N}$ or to zero. Thus Eq. (D7) is still satisfied because $\mathcal{N}$ is a nonevent generator. On the other hand, $\mathcal{N}_{\bar{x} x}=\Pi_{\bar{x}} \mathcal{N} \Pi_{x}$, and thus all of its diagonal elements are zero. Its off-diagonal elements are either equal to those of $\mathcal{N}$, which are non-negative, or zero. Thus Eq. (D8) is satisfied, proving that $\mathcal{N}_{\bar{x} x}$ is an event generator. Finally, the sum of the generators satisfies [from Eq. (E20)]

$$
\begin{aligned}
& \mathcal{N}_{x x}+\mathcal{N}_{\bar{x} x}=\Pi_{\bar{x}} \mathcal{N} \Pi_{x}+\Pi_{x} \mathcal{N} \Pi_{x}=\mathcal{N} \Pi_{x}, \\
& \text { because } \Pi_{\bar{x}}+\Pi_{x}=\mathbb{1} \text {. }
\end{aligned}
$$

Thus the sum of the two generators is the original nonevent generator $\mathcal{N}$ with some of its columns set to zero (those outside the support of $\Pi_{x}$ ). Thus adding the column sums of $\mathcal{N}_{x x}$ and $\mathcal{N}_{\bar{x} x}$ gives either the corresponding column sum of $\mathcal{N}$, or zero. In either case, Eq. (D9) is satisfied, completing the conditions that determine that $\left\{\mathcal{N}_{x x}, \mathcal{N}_{\bar{x} x}\right\}$ is a pair of stochastic generators.

To prove that the sequence is an independent one (Definition 14), one has to show that both of the nonevent generators in the sequence, the $\mathcal{N}_{\bar{x} x}$, are rank-1. From Eq. (E21), one observes that $\mathcal{N}_{01}$ has a single nonzero row, while $\mathcal{N}_{10}$ has a single nonzero column. Thus Definition 14 is satisfied.

To demonstrate that each $v_{n, x}(t) \in S_{x}$, we express them as (for all cases except $n=x=0$ ) the solution to their differential equations [Eq. (E27)],

$$
\begin{aligned}
v_{n, x}(t) & =v_{n, x}(t=0)+\int_{0}^{t} \frac{d}{d t^{\prime}} v_{n, x}\left(t^{\prime}\right) d t^{\prime} \\
& =\int_{0}^{t} \mathcal{N}_{x x} v_{n, x}\left(t^{\prime}\right)+\mathcal{N}_{x \bar{x}} v_{n-\bar{x}, \bar{x}}\left(t^{\prime}\right) d t^{\prime} \\
& =\int_{0}^{t} \Pi_{x} \mathcal{N} \Pi_{x} v_{n, x}\left(t^{\prime}\right)+\Pi_{x} \mathcal{N} \Pi_{\bar{x}} v_{n-\bar{x}, \bar{x}}\left(t^{\prime}\right) d t^{\prime}
\end{aligned}
$$

using the definition of the $\mathcal{N}_{x y}$ [Eq. (E20)], and since the initial states are all zero vectors except for $n=x=0$. Left multiplying the above expression by the projects $\Pi_{x}$ and $\Pi_{\bar{x}}$, respectively, recovers the statement of the lemma.

For the special case $n=x=0$, the initial state $v_{0,0}(0)$ is the canonical state $\mathbf{e}_{0}$ and thus satisfies the lemma. Furthermore, the derivative of the state is proportional only to $\mathcal{N}_{00}=\Pi_{0} \mathcal{N} \Pi_{0}$. The proof thus follows in an analogous manner.

Lemma 15: (Decomposing the path-specific delay functions with respect to the path-generating delay functions.) The path-specific delay functions $\xi_{n, x}(t)$ (Definition 23) are sequential convolutions of the path-generating delay functions $\Theta_{x}$ (Definition 26),

$$
\xi_{n, x}(t)=\left(\Theta_{1} * \Theta_{0} * \Theta_{1} * \Theta_{0} * \cdots * \Theta_{x}\right)(t),
$$

where $\Theta_{1}$ appears $n+x$ times and $\Theta_{0}$ appears $n$ times.

Proof. As Lemma 14 has proven that $\xi_{n, x}(t)$ are the delay functions of an independent Markovian sequence, we may apply Lemma 11, to express them as

$$
\xi_{n, x}(t)=\left(v_{0,1} * v_{1,0} * v_{1,1} * v_{2,0} * \cdots * v_{n, x}\right)(t),
$$

where the subevent delay functions $v_{n, x}$ are defined in Definition 17, and for the present sequence, take on the form

$$
v_{n, x}(t)=\left\|\mathcal{N}_{x \bar{x}} e^{\mathcal{N}} \overline{\bar{x} x} t \mathbf{w}_{n, \bar{x}}\right\|_{\Sigma},
$$

where $\mathbf{w}_{n, \bar{x}}$ is the reset state (Definition 15) for the event $\{n, x\}$ in the sequence.

Consider the reset state $\mathbf{w}_{n, 0}$. This is defined to be (Definition 15) the unique normalized state that is proportional to every column of the corresponding generator in the sequence, which in this case is $\mathcal{N}_{01}$ (see Lemma 14). From the definition of $\mathcal{N}_{x y}$ [see Eq. (E21)], we conclude that this is simply the canonical state $\mathbf{e}_{0}$, and thus $\mathbf{w}_{n, 0}=$ $\mathbf{u}_{0}$, the path-generating reset state defined in Definition 25 .

In a similar manner, the reset state $\mathbf{w}_{n, 1}$ is the unique normalized state proportional to every column of $\mathcal{N}_{10}$. This operator has only a single nonzero column, and the corresponding normalized state has already been defined to be the other path-generating reset state, $\mathbf{u}_{1}$ (Definition 25).

Thus, returning to Eq. (E39), we see that the subevent delay functions $v_{n, x}(t)$ are in fact, independent of $n$, and equal to the path-generating delay functions $\Theta_{x}$ defined in Definition 26. Substituting these back into Eq. (E37), we recover the statement of the lemma. 
Lemma 16: [Explicit form and properties of the pathgenerating delay function $\Theta_{1}$ (Definition 26) and tickgenerating delay function $\Gamma_{1}$ (Definition 27).]

$$
\begin{aligned}
& \Theta_{1}(t)=A e^{-g t}, \\
& \Gamma_{1}(t)=B e^{-g t},
\end{aligned}
$$

where $A \geq 0$ is the sum of the first (and only nonzero) column of $\overline{\mathcal{N}}_{10}, B \geq 0$ is the only (possibly) nonzero element of $\mathcal{T}_{00}$, and $g \geq 0$ is the negation of the singular nonzero element in $\mathcal{N}_{00}$. [see Eq. (E21)]. If $g=0$, then $\Theta_{1}(t)=$ $\Gamma_{1}(t)=0$ for all $t$. Denoting the moments (Definition 6) of $\Theta_{1}$ by $\left\{Q_{1}, \mu_{1}, \chi_{1}\right\}$ and those of $\Gamma_{1}$ by $\left\{Q_{3}, \mu_{3}, \chi_{3}\right\}$, they are (in the case $g>0$ )

$$
\begin{aligned}
Q_{1} & =\frac{A}{g}, \\
Q_{3} & =\frac{B}{g}, \\
\mu_{1} & =\mu_{3}=\frac{1}{g}, \\
\chi_{1} & =\chi_{3}=\frac{2}{g^{2}},
\end{aligned}
$$

and their $\gamma$ values and accuracies $R$ [Definition 7 and $E q$. (B6)] are therefore

$$
\begin{aligned}
\gamma_{1} & =\gamma_{3}=2, \\
R\left[\Theta_{1}\right] & =R\left[\Gamma_{1}\right]=1 .
\end{aligned}
$$

Proof. Via the definition of $\Theta_{1}$ in Definition 26, and since $\mathbf{u}_{0}=\mathbf{e}_{0}$ (Definition 25),

$$
\Theta_{1}(t)=\left\|\mathcal{N}_{10} e^{\mathcal{N}_{00} t} \mathbf{e}_{0}\right\|_{\Sigma}
$$

However, the operator $\mathcal{N}_{00}$ [see Eq. (E21)] has only a single nonzero element, on the diagonal, and corresponding to $\mathbf{e}_{0}$. We label this element by $-g$, where $g \geq 0$ (recall that the diagonal elements of $\mathcal{N}$ are nonpositive (Definition 12). One thus simplifies the action of $e^{\mathcal{N}_{00} t}$ on $\mathbf{e}_{0}$, obtaining

$$
\begin{aligned}
\Theta_{1}(t) & =\left\|\mathcal{N}_{10} e^{-g t} \mathbf{e}_{0}\right\|_{\Sigma} \\
& =A e^{-g t},
\end{aligned}
$$

where $A=\left\|\mathcal{N}_{10} \mathbf{e}_{0}\right\|_{\Sigma}$ is the sum of the first column of $\mathcal{N}_{10}$.

If $g=0$, then from the definition of stochastic generators, Definition 12 and Corollary 2, it follows that the entire first column of $\mathcal{N}$ is zero, and thus $A$ is also zero, leading to $\Theta_{1}$ being the zero function.
In a similar manner, using the definition of $\Gamma_{1}$ (Definition 27),

$$
\Gamma_{1}=\left\|\mathcal{T}_{00} e^{\mathcal{N}_{00} t} \mathbf{e}_{0}\right\|_{\Sigma}=B e^{-g t},
$$

where $B$ is the single (possibly) nonzero element in $\mathcal{T}_{00}$. If $g=0$, then it follows from the fact that $\left\{\mathcal{N}_{00}, \mathcal{T}_{00}\right\}$ is also a pair of stochastic generators (Definition 12), that $B=0$ as well, and therefore $\Gamma_{1}$ is the zero function in this case.

The rest of the lemma follows from the direct application of the definition of the moments (Definition 6), the accuracy $R$ (Definition 7), and the $\gamma$ value [Eq. (B6)], in the case that $g>0$.

Corollary 7: The partial norm (Definition 8) of the pathgeneration delay function $\Theta_{1}$ is strictly smaller than 1 for all $t \geq 0$.

Proof. From the explicit form of $\Theta_{1}$ (Lemma 16), we can calculate its partial norm (Definition 8) explicitly,

$$
P_{t}\left[\Theta_{1}\right]=\int_{0}^{t} \Theta_{1}(t) d t= \begin{cases}0 & \text { if } g=0, \\ \frac{A}{g}\left(1-e^{-g t}\right) & \text { if } g>0 .\end{cases}
$$

The case $g=0$ satisfies the corollary trivially. For the case $g>0$, note that

$$
P_{t}\left[\Theta_{1}\right]=\frac{A}{g}\left(1-e^{-g t}\right)=Q_{1}\left(1-e^{-g t}\right)<Q_{1} \quad \forall t,
$$

where $Q_{1}$ is the zeroth moment of $\Theta_{1}$ (Lemma 16), and is itself upper bounded by 1 (see Definition 5). Thus the partial norm is strictly less than 1 for all $t$.

Lemma 17: The delay functions $\Theta_{0}$ (Definition 26) and $\Gamma_{0}$ (Definition 27) can be generated by $d$-1-dimensional clocks.

Proof. We prove the statement for $\Theta_{0}$, the proof for $\Gamma_{0}$ is analogous. From Definition 26,

$$
\Theta_{0}(t)=\left\|\mathcal{N}_{01} e^{\mathcal{N}_{11} t} \mathbf{u}_{1}\right\|_{\Sigma} .
$$

While the expression above appears to involve the entire vector space $\mathbb{R}^{d}$, in fact, one can generate the same delay function with only $d$-1-dimensional objects, as we proceed to show.

First off, take the event generator $\mathcal{N}_{01}$. From Eq. (E21), we observe that only the first row is nonzero, and of this row, the first element is zero. Consider the modified event generator $\mathcal{N}_{01}^{\prime}$, formed by swapping the first row with 
any other. By Lemma 9, this leaves the delay function $\Theta_{0}(t)$ unchanged. The entire first row and column of the modified generator are zero. Next, we note that $\mathcal{N}_{11}$ by construction [see Eq. (E21)] already has a zero first row and column. Thus both $\mathcal{N}_{11}$ and $\mathcal{N}_{01}^{\prime}$ act trivially on the canonical state $\mathbf{e}_{0}$.

Finally, $\mathbf{u}_{1}$ (Definition 25) is constructed from the first column of $\mathcal{N}_{10}$, that has a zero element at the top [see Eq. (E21)], and thus $\mathbf{u}_{1}$ has no component from the canonical state $\mathbf{e}_{0}$. Thus we may simply remove this space entirely from $\mathbf{u}_{1}$, and correspondingly from the operators $\mathcal{N}_{11}$ and $\mathcal{N}_{01}^{\prime}$, and still generate the same delay function $\Theta_{0}$.

Corollary 8: Assuming that the accuracy of a single tick of a $d$-1-dimensional clock is upper bound by $R \leq d-$ 1 , as is done during this proof, one can lower bound the second moments of $\Theta_{0}$ and $\Gamma_{0}$ with respect to their first moments,

$$
\begin{aligned}
& \chi_{0} \geq \mu_{0}^{2}\left(1+\frac{1}{d-1}\right), \\
& \chi_{2} \geq \mu_{2}^{2}\left(1+\frac{1}{d-1}\right),
\end{aligned}
$$

if the first moments $\mu_{0}$ and $\mu_{2}$ do not diverge.

Proof. From Lemma 17, we know that both $\Theta_{0}$ and $\Gamma_{0}$ can be generated by $d$-1-dimensional clocks, but we assume the accuracy $R$ (Definition 7) of these clocks to be upper bound by $R \leq d-1$. The corollary then follows from the definition of the $\gamma$ value [Eq. (B6)] and its relationship with the accuracy $R$.

Lemma 18: (Recovering the clock state and delay function from the path-specific states and component delay functions.) The state of the clock $V(t)$ corresponding to the first tick [Eq. (E13)] is the series sum of the path-specific states (Definition 22),

$$
V(t)=\sum_{n=0}^{\infty} \sum_{x \in\{0,1\}} v_{n, x}(t) .
$$

Furthermore, the delay function $\tau(t)$ of a single tick of the clock [Eq. (E13)] is the series sum of the component delay functions (Definition 24),

$$
\tau(t)=\tau_{0,1}(t)+\sum_{n=1}^{\infty} \sum_{x \in\{0,1\}} \tau_{n, x}(t) .
$$

Proof. Consider the following sequence of states, $\left\{v_{n, x}^{(M)}(t)\right\}$, defined for $x \in\{0,1\}$ and $n \in\{0,1, \ldots, M\}$, where
$M \in \mathbb{N}^{+}$, and whose initial states and dynamics are identical to those of the path-specific states $v_{n, x}(t)$ (see Definition 22) except for the last one $v_{M, 1}^{(M)}(t)$, that evolves as

$$
\frac{d}{d t} v_{M, 1}^{(M)}=\mathcal{N}_{10} v_{M, 0}^{(M)}+\mathcal{N} v_{M, 1}^{(M)}
$$

where the last term above differentiates it from the dynamics of the original sequence, by including the entire nonevent generator $\mathcal{N}$ rather than only $\mathcal{N}_{11}$. Intuitively this corresponds to interrupting the sequence at $n=M, x=1$ by stopping the flow of population to further states. It is straightforward to verify that this too is an independent Markovian sequence of events (Definition 14).

Since the initial states and dynamical equations of all but the last state in the sequence $v_{n, x}^{(M)}$ are identical to those of the sequence $v_{n, x}$, and because the dynamics of every state in a Markovian sequence depend only on the previous states in the sequence, it follows that $v_{n, x}^{(M)}(t)=v_{n, x}(t)$ for all $x$ and $M$ except for the final state $v_{M, 1}^{(M)}(t)$. Furthermore, this also implies that the corresponding delay functions of the sequence of states $v_{n, x}^{(M)}$ are the path-specific delay functions $\xi_{n, x}$ (Definition 23), upto and including $n=M$, $x=1$.

Consider the vector $V^{(M)}$, defined as the sum

$$
V^{(M)}(t)=\sum_{n=0}^{M} \sum_{x \in\{0,1\}} v_{n, x}^{(M)}(t),
$$

that we proceed to show is equal to $V(t)$, the state of the clock for a single tick [Eq. (E13)]. For $t=0$, by construction $V^{(M)}(0)=V(0)$. For $t \geq 0$, the dynamics of the sum is given by [Eqs. (E27) and (E55)]

$$
\begin{aligned}
\frac{d}{d t} V^{(M)}(t)= & \sum_{n=0}^{M} \sum_{x \in\{0,1\}} \frac{d}{d t} v_{n, x}^{(M)}(t) \\
= & \sum_{n=0}^{M-1} \sum_{x \in\{0,1\}}\left(\mathcal{N}_{x x}+\mathcal{N}_{\bar{x} x}\right) v_{n, x}^{(M)}(t) \\
& +\left(\mathcal{N}_{00}+\mathcal{N}_{10}\right) v_{M, 0}^{(M)}(t)+\mathcal{N} v_{M, 1}^{(M)}(t) \\
= & \sum_{n=0}^{M-1} \sum_{x \in\{0,1\}}\left(\mathcal{N}_{x x}+\mathcal{N}_{\bar{x} x}\right) v_{n, x}(t) \\
& +\left(\mathcal{N}_{00}+\mathcal{N}_{10}\right) v_{M, 0}(t)+\mathcal{N} v_{M, 1}^{(M)}(t) .
\end{aligned}
$$

We may use the fact that each $v_{n, x}(t) \in S_{x}$ for all $t \geq 0$ (Lemma 14), together with the definition of the generators 
$\mathcal{N}_{x y}$ [Eq. (E20)] to conclude that for all $n$ and $x$,

$$
\left(\mathcal{N}_{\bar{x} \bar{x}}+\mathcal{N}_{x \bar{x}}\right) v_{n, x}(t)=\mathbf{0} .
$$

Adding these trivial zeros to Eq. (E59), and using the decomposition of $\mathcal{N}$ [Eq. (E20)]

$$
\begin{aligned}
\frac{d}{d t} V^{(M)}(t) & =\sum_{n=0}^{M} \sum_{x \in\{0,1\}} \mathcal{N} v_{n, x}^{(M)}(t) \\
& =\mathcal{N} V^{(M)}(t), \\
\therefore V^{(M)}(t) & =e^{\mathcal{N} t} V^{(M)}(0)=e^{\mathcal{N} t} V(0)=V(t) .
\end{aligned}
$$

Finally, we investigate the limit $M \rightarrow \infty$ of $V^{(M)}(t)$,

$$
\begin{aligned}
& \lim _{M \rightarrow \infty} V^{(M)}(t) \\
& =\lim _{M \rightarrow \infty}\left(\sum_{n=0}^{M-1} \sum_{x \in\{0,1\}} v_{n, x}(t)+v_{M, 0}(t)+v_{M, 1}^{(M)}(t)\right) \\
& =\sum_{n=0}^{\infty} \sum_{x \in\{0,1\}} v_{n, x}(t)+\lim _{M \rightarrow \infty} v_{M, 1}^{(M)}(t) .
\end{aligned}
$$

To prove that the state $v_{M, 1}^{(M)}(t)$ goes to zero, we first apply Lemma 11 to express it as

$$
v_{M, 1}^{(M)}(t)=\int_{0}^{t} e^{\mathcal{N}\left(t-t^{\prime}\right)} \mathbf{w}_{M, 1} \xi_{M, 1}\left(t^{\prime}\right)\left(t^{\prime}\right) d t^{\prime}
$$

where $\mathbf{w}_{M, 1}$ is the event reset state (Definition 15) for the $n=M, x=1$ event, that has already been proven to be equal to $\mathbf{u}_{1}$ [see discussion after Eq. (E39)]. Calculating the norm (Definition 11) of the above,

$$
\left\|v_{M, 1}(t)\right\|_{\Sigma}=\int_{0}^{t}\left\|e^{\mathcal{N}_{11}\left(t-t^{\prime}\right)} \mathbf{u}_{1}\right\|_{\Sigma} \xi_{M, 1}\left(t^{\prime}\right) d t^{\prime}
$$

However, the term in the e sum above is a population vector for all $t-t^{\prime} \geq 0$ (see Lemma 6), and thus the norm is upper bounded by 1 , leading to

$$
\left\|v_{M, 1}(t)\right\|_{\Sigma} \leq \int_{0}^{t} \xi_{M, 1}\left(t^{\prime}\right) d t^{\prime}=P_{t}\left[\xi_{M, 1}\right]
$$

where the partial norm $P_{t}[\cdot]$ is defined in Definition 8 . As $\xi_{n, x}$ is itself a convolution of delay functions (Lemma 15), we can apply Lemma 3, to obtain

$$
\begin{aligned}
\left\|v_{M, 1}(t)\right\|_{\Sigma} & \leq\left(P_{t}\left[\Theta_{1}\right]\right)^{M+1}\left(P_{t}\left[\Theta_{0}\right]\right)^{M} \\
& \leq\left(P_{t}\left[\Theta_{1}\right]\right)^{M+1},
\end{aligned}
$$

using the fact that the partial norm is upper bounded by 1 .
However, from Corollary 7, the partial norm of $\Theta_{1}$ is strictly less than 1 for all $t \geq 0$, and thus

$$
\lim _{M \rightarrow \infty}\left\|v_{M, 1}(t)\right\|_{\Sigma}=0
$$

which in turn implies that every element of $v_{M, 1}(t)$ must individually go to zero. Combining this with Eqs. (E63) and (E65), we recover

$$
V(t)=\sum_{n=0}^{\infty} \sum_{x \in\{0,1\}} v_{n, x}(t) .
$$

The proof of the second statement of the lemma, concerning the delay function, proceeds in an analogous manner. Consider the following sequence of delay functions, $\left\{\tau^{(M)}(t)\right\}$, defined with respect to the sequence of states $v_{n, x}^{(M)}(t)$ as

$$
\tau_{n, x}^{(M)}(t)=\left\|\mathcal{T}_{0 \bar{x}} v_{n-\bar{x}, \bar{x}}^{(M)}(t)\right\|_{\Sigma},
$$

for all $n, x$ from $n=0, x=1$ up to and including $n=$ $M, x=1$. Finally, the end delay function of the sequence is defined as

$$
\tau_{M+1,0}^{(M)}(t)=\left\|\mathcal{T} v_{M, 1}^{(M)}(t)\right\|_{\Sigma} .
$$

Note immediately that since $v_{n, x}^{(M)}(t)=v_{n, x}(t)$ for all the states except $v_{M, 1}^{(M)}(t)$, it follows that $\tau_{n, x}^{(M)}(t)=\tau_{n, x}(t)$ (Definition 23) except for the final delay function $\tau_{M+1,0}^{(M)}(t)$.

Consider the sum

$$
f^{(M)}(t)=\tau_{0,1}^{(M)}(t)+\sum_{n=1}^{M} \sum_{x \in\{0,1\}} \tau_{n, x}^{(M)}(t)+\tau_{M+1,0}^{(M)}(t),
$$

which we proceed to show is equal to the delay function $\tau(t)$ of a single tick of the clock, Eq. (E13).

$$
\begin{aligned}
f^{(M)}(t)= & \left\|\mathcal{T}_{00} v_{0,0}^{(M)}(t)\right\|_{\Sigma}+\sum_{n=1}^{M-1} \sum_{x \in\{0,1\}}\left\|\mathcal{T}_{0 \bar{x}} v_{n-\bar{x}, \bar{x}}^{(M)}(t)\right\|_{\Sigma} \\
& +\left\|\mathcal{T}_{00} v_{M, 0}^{(M)}(t)\right\|_{\Sigma}+\left\|\mathcal{T} v_{M, 1}^{(M)}(t)\right\|_{\Sigma} \\
= & \left\|\mathcal{T}_{00} v_{0,0}(t)\right\|_{\Sigma}+\sum_{n=1}^{M} \sum_{x \in\{0,1\}}\left\|\mathcal{T}_{0 \bar{x}} v_{n-\bar{x}, \bar{x}}(t)\right\|_{\Sigma} \\
& +\left\|\mathcal{T}_{00} v_{M, 0}(t)\right\|_{\Sigma}+\left\|\mathcal{T} v_{M, 1}^{(M)}(t)\right\|_{\Sigma}
\end{aligned}
$$


Using the definition of $\mathcal{T}_{x y}=\Pi_{x} \mathcal{T} \Pi_{y}$ [Eq. (E23)], and also the fact that each $v_{n, x} \in S_{x}$ (Lemma 14), we can trivially add $\mathcal{T}_{0 \bar{x}} v_{n, x}^{(M)}=\mathbf{0}$ to any expression, and doing so to the above equation results in

$$
\begin{aligned}
f^{(M)}(t) & =\left\|\sum_{n=0}^{M-1} \sum_{x \in\{0,1\}}\left(\mathcal{T}_{0 x}+\mathcal{T}_{0 \bar{x}}\right) v_{n, x}^{(M)}(t)\right\|_{\Sigma}+\left\|\left(\mathcal{T}_{0 x}+\mathcal{T}_{0 \bar{x}}\right) v_{M, 0}^{(M)}(t)\right\|_{\Sigma}+\left\|\mathcal{T} v_{M, 1}^{(M)}(t)\right\|_{\Sigma} \\
& =\left\|\mathcal{T} V^{(M)}(t)\right\|_{\Sigma}=\|\mathcal{T} V(t)\|_{\Sigma}=\tau(t) .
\end{aligned}
$$

Thus $f^{(M)}(t)$ is the delay function of a single tick of the clock [Eq. (E13)]. To complete the proof, we investigate the limit as $M \rightarrow \infty$,

$$
\begin{aligned}
\lim _{M \rightarrow \infty} f^{(M)} & =\lim _{M \rightarrow \infty}\left(\tau_{0,1}^{(M)}(t)+\sum_{n=1}^{M} \sum_{x \in\{0,1\}} \tau_{n, x}^{(M)}(t)+\tau_{M+1,0}^{(M)}(t)\right) \\
& =\lim _{M \rightarrow \infty}\left(\tau_{0,1}(t)+\sum_{n=1}^{M} \sum_{x \in\{0,1\}} \tau_{n, x}(t)\right)+\lim _{M \rightarrow \infty} \tau_{M+1,0}^{(M)}(t) \\
& =\sum_{n=0}^{\infty} \sum_{x \in\{0,1\}} \tau_{n, x}(t)+\lim _{M \rightarrow \infty}\left\|\mathcal{T} v_{M, 1}^{(M)}(t)\right\|_{\Sigma} .
\end{aligned}
$$

But the remaining term on the right goes to zero, as we have already proved that $v_{M, 1}^{(M)}(t)$ goes to zero [see Eq. (E71)], and $\mathcal{T}$ is a bounded linear operator. From the above expression and Eq. (E79), we recover

$$
\tau(t)=\sum_{n=0}^{\infty} \sum_{x \in\{0,1\}} \tau_{n, x}(t),
$$

which completes the proof of the lemma.

\section{c. The moments of the delay function of a single tick of the clock}

We proceed by calculating the moments of the delay function of a single tick of a clock by using the pathspecific delay functions and component delay functions.

Consider the component delay function (Definition 24),

$$
\begin{aligned}
\tau_{n, x}(t) & =\left\|\mathcal{T}_{0 \bar{x}} v_{n-\bar{x}, \bar{x}}(t)\right\|_{\Sigma} \\
& =\left\|\int_{0}^{t} \mathcal{T}_{0 \bar{x}} e^{\mathcal{N}_{\bar{x} \bar{x}}\left(t-t^{\prime}\right)} \mathbf{u}_{\bar{x}} \xi_{n-\bar{x}, \bar{x}}\left(t^{\prime}\right) d t^{\prime}\right\|_{\Sigma},
\end{aligned}
$$

where we apply Lemma 11 to express the path-specific state $v_{n-\bar{x}, \bar{x}}(t)$ with respect to its corresponding pathspecific delay function, as they form an independent
Markovian sequence (Lemma 14). Continuing, we simplify the above to

$$
\begin{aligned}
\tau_{n, x}(t) & =\int_{0}^{t}\left\|\mathcal{T}_{0 \bar{x}} e^{\mathcal{N}_{\bar{x} \bar{x}}\left(t-t^{\prime}\right)} \mathbf{u}_{\bar{x}}\right\|_{\Sigma} \xi_{n-\bar{x}, \bar{x}}\left(t^{\prime}\right) d t^{\prime} \\
& =\int_{0}^{t} \Gamma_{x}\left(t-t^{\prime}\right) \xi_{n-\bar{x}, \bar{x}}\left(t^{\prime}\right) d t^{\prime}
\end{aligned}
$$

using the definition of the tick-generating delay functions $\Gamma_{x}$ (Definition 27). Finally,

$$
\begin{aligned}
\tau_{n, x}(t) & =\left(\Gamma_{x} * \xi_{n-\bar{x}, \bar{x}}\right)(t) \\
& =\left(\Gamma_{x} * \Theta_{1} * \Theta_{0} * \Theta_{1} * \Theta_{0} * \cdots * \Theta_{\bar{x}}\right)(t),
\end{aligned}
$$

using Lemma 15. In the above convolution, $\Theta_{1}$ appears $n$ times, and $\Theta_{0}$ appears $n-\bar{x}$ times.

We can now calculate the moments of the component delay function $\tau_{n, x}$ using Eq. (B8) for a convolution of a sequence of delay functions. The moments (Definition 6) of $\Theta_{1}$ and $\Gamma_{1}$ have already been labeled and calculated in Lemma 16. For now, we label them (in order of zeroth, first, and second moments) as $\left\{Q_{1}, \mu_{1}, \chi_{1}\right\}$ for $\Theta_{1}$ and $\left\{Q_{3}, \mu_{1}, \chi_{1}\right\}$ for $\Gamma_{1}$ (the first and second moments of these two delay functions were proven equal in Lemma 16). The moments of $\Theta_{0}$ (Definition 26) are denoted as $\left\{Q_{0}, \mu_{0}, \chi_{0}\right\}$ and the moments of $\Gamma_{0}$ (Definition 27) by $\left\{Q_{2}, \mu_{2}, \chi_{2}\right\}$.

Finally, denoting the zeroth, first, and second moments of the component delay function $\tau_{n, x}(t)$ by $Q_{n, x}, \mu_{n, x}$ and 
$\chi_{n, x}$, respectively, from Eq. (B8),

$$
\begin{aligned}
Q_{n, 0}= & Q_{1}^{n} Q_{0}^{n-1} Q_{2}, \\
\mu_{n, 0}= & n \mu_{1}+(n-1) \mu_{0}+\mu_{2}, \\
\chi_{n, 0}= & n \chi_{1}+(n-1) \chi_{0}+\chi_{2}+n(n-1) \mu_{1}^{2} \\
& +(n-1)(n-2) \mu_{0}^{2}+2 n(n-1) \mu_{1} \mu_{0} \\
& +2 n \mu_{1} \mu_{2}+2(n-1) \mu_{0} \mu_{2}, \\
Q_{n, 1}= & Q_{1}^{n} Q_{0}^{n} Q_{3}, \\
\mu_{n, 1}= & (n+1) \mu_{1}+n \mu_{0}, \\
\chi_{n, 1}= & (n+1) \chi_{1}+n \chi_{0}+n(n+1) \mu_{1}^{2} \\
& +n(n-1) \chi_{0}^{2}+2 n(n+1) \mu_{1} \mu_{0} .
\end{aligned}
$$

We are now in a position to calculate the accuracy of a single tick of the clock. First off, we split the delay function of a single tick of the clock into two parts, with respect to the some over component delay functions,

$$
\begin{aligned}
\tau(t) & =\tau_{0,1}(t)+\sum_{n=0}^{\infty} \sum_{x \in\{0,1\}} \tau_{n, x}(t) \\
& =\sum_{n=1}^{\infty} \tau_{n, 0}(t)+\sum_{n=0}^{\infty} \tau_{n, 1}(t) \\
& =\tau^{(1)}(t)+\tau^{(0)}(t) .
\end{aligned}
$$

We may understand the above division in the following manner. The component delay function $\tau_{n, x}$ is generated by $\mathcal{T}_{0 \bar{x}}$ (see Definition 24 ), and thus represents a tick being generated from the $S_{\bar{x}}$ subspace of the clock vector space. Thus the two $\tau^{(x)}(t)$ above each correspond to the delay function of a single tick generated from $S_{x}$.

From Lemma 4, the accuracy of the sum of two delay functions is upper bound by the maximum accuracy from among the two, and we proceed to bound each of the accuracies individually.

For $\tau^{(0)}$ (that corresponds to the tick arising from the $S_{0}$ subspace), we find the zeroth, first, and second moments of the entire tick density to be (on application of Definition 6),

$$
\begin{aligned}
Q^{(0)} & =\sum_{n=0}^{\infty} Q_{n, 1}=\frac{Q_{3}}{1-Q_{1} Q_{0}}, \\
\mu^{(0)} & =\frac{\sum_{n=0}^{\infty} Q_{n, 1} \mu_{n, 1}}{Q^{(0)}}=\frac{Q_{0} Q_{1} \mu_{0}+\mu_{1}}{1-Q_{0} Q_{1}}, \\
\chi^{(0)} & =\frac{\sum_{n=0}^{\infty} Q_{n, 1} \chi_{n, 1}}{Q^{(0)}}=\frac{\left(Q_{0} Q_{1} \mu_{0}+\mu_{1}\right)^{2}+Q_{0} Q_{1} \chi_{0}\left(1-Q_{0} Q_{1}\right)}{\left(1-Q_{0} Q_{1}\right)^{2}},
\end{aligned}
$$

where we replace $\chi_{1}=2 \mu_{1}^{2}$ [Eq. (E42)] to simplify the expressions. Proceeding, we find that the accuracy is given by [Eq. (B6)]

$$
R\left[\tau^{(0)}\right]=\left(\frac{\chi^{(0)}}{\left(\mu^{(0)}\right)^{2}}-1\right)^{-1}=\frac{\left(Q_{0} Q_{1} \mu_{0}+\mu_{1}\right)^{2}}{\left(Q_{0} Q_{1} \mu_{0}+\mu_{1}\right)^{2}+Q_{0} Q_{1} \chi_{0}\left(1-Q_{0} Q_{1}\right)}
$$

As $Q_{0} Q_{1}$ is the product of the zeroth moments of delay functions and thus $\leq 1$, we see that the accuracy decreases monotonically with respect to $\chi_{0}$. As such, we may upper bound it by replacing $\chi_{0}$ by its minimum value using Corollary 8. Furthermore, noting that the moments $Q_{0}$ and $Q_{1}$ appear only as the product, and that $\mu_{0}$ and $\mu_{1}$ affect only the accuracy via their relative ratio, we can simplify the expression to

$$
R\left[\tau^{(0)}\right] \leq \frac{(d-1)(1+p r)^{2}}{d+d p r(2+r)-(1+p r)^{2}},
$$

where $p=Q_{0} Q_{1} \in(0,1]$ and $r=\mu_{0} / \mu_{1} \in(0, \infty)$. One can analytically optimize the above expression with respect to $p$ and $r$, and we find that the maximal value of the accuracy is

$$
R\left[\tau^{(0)}\right] \leq 1,
$$

which is only the accuracy of a one-dimensional clock, and not very interesting. 
On the other hand, consider the delay function $\tau^{(1)}(t)$, that corresponds to the tick being generated from $S_{1}$. We find the moments of the delay function to be

$$
\begin{aligned}
Q^{(1)} & =\sum_{n=0}^{\infty} Q_{n, 0}=\frac{Q_{1} Q_{2}}{1-Q_{0} Q_{1}}, \\
\mu^{(1)} & =\frac{\sum_{n=0}^{\infty} Q_{n, 0} \mu_{n, 0}}{Q^{(1)}}=\frac{Q_{0} Q_{1} \mu_{0}+\mu_{1}}{1-Q_{0} Q_{1}}+\mu_{2}, \\
\chi^{(1)} & =\frac{\sum_{n=0}^{\infty} Q_{n, 0} \chi_{n, 0}}{Q^{(1)}}=\frac{2\left(Q_{0} Q_{1} \mu_{0}+\mu_{1}\right)\left[\mu_{1}+Q_{0} Q_{1}\left(\mu_{0}-\mu_{2}\right)+\mu_{2}\right]+\left(1-Q_{0} Q_{1}\right)\left[Q_{0} Q_{1} \chi_{0}+\left(1-Q_{0} Q_{1}\right) \chi_{2}\right]}{\left(1-Q_{0} Q_{1}\right)^{2}} .
\end{aligned}
$$

Here too, the second moment is seen to increase monotonically with respect to both $\chi_{0}$ and $\chi_{2}$. As the accuracy itself is inversely related to the second moment, we may substitute the minimum $\chi_{0}$ and $\chi_{2}$ by their minimum values using Corollary 8 to upper bound the accuracy. Furthermore, we replace $Q_{0} Q_{1}=p \in(0,1], \mu_{0} / \mu_{1}=r \in(0, \infty)$ and $\mu_{2} / \mu_{1}=$ $s \in(0, \infty)$ to find that the bound on the accuracy takes on the form

$$
R\left[\tau^{(1)}\right]=\left(\frac{\chi^{(1)}}{\left(\mu^{(1)}\right)^{2}}-1\right)^{-1} \leq \frac{(d-1)(1+p(r-s)+s)^{2}}{d+d p r(2+r)-(1-p r)^{2}+(1-p)^{2} s^{2}} .
$$

One may analytically optimize the above expression with respect to $\{p, r, s\}$, and doing so returns the maximal value

$$
R\left[\tau^{(1)}\right] \leq d
$$

Thus the accuracy of $\tau^{(1)}$, and thus $\tau$, the delay function of a single tick of a $d$-dimensional reset clock, is upper bound by $d$. By induction the result follows for all $d$. From Sec. E 2 a, we can then conclude that the accuracy of the $n$th tick of the reset clock (and thus any clock, from Theorem 4) is upper bound by $n d$, completing the statement of the theorem.

\section{APPENDIX F: QUANTUM CLOCKS - PROOF OF THEOREM ??}

The purpose of this section is to prove Theorem 2 in the main text. For the sake of generality, the version of the theorem, which we derive, Theorem 6, is more general than Theorem 2, which has not been stated in the main text since it requires some more technical definitions. After summarizing the setup in the example Sec. II C, we start by introducing the quasi-ideal clock and stating the necessary theorems about its dynamical properties from [8]. Then we summarize the exact form of the potential to which Theorem 6 applies. Then we proceed by proving the necessary lemmas, and mathematical statements before proving Theorem 6 in Sec. F 8.

\section{Setup}

In this section, we briefly recall the important results from the example of a quantum clock in Sec. II C, since they will form the starting point of the proof.

The probability of not getting a tick in time interval $[0, t]$, is $\operatorname{tr}[\rho(t)]$, where $\left|\bar{\psi}_{t}\right\rangle$ is given by Eq. (22), namely

$$
\rho(t):=\left|\bar{\psi}_{t}\right\rangle\left\langle\bar{\psi}_{t}\right|=e^{-i t H} \rho_{0} e^{i t H^{\dagger}},
$$

where $\rho_{0}=\left|\psi_{0}\right\rangle\left\langle\psi_{0}\right|$ is the initial state, and

$$
H=\hat{H}_{C}-i \hat{V}_{C}
$$

describes the evolution of the system with $\hat{H}_{C}$ being the Hamiltonian and $\hat{V}_{C}$ the potential. The probability of getting the first tick in the infinitesimal time step $\delta>0$ is then

$$
\begin{aligned}
P(t)= & 2 \operatorname{tr}\left[\hat{V}_{C} \rho(t)\right]=\frac{d}{d t}\{1-\operatorname{tr}[\rho(t)]\}=-\frac{d}{d t} \operatorname{tr}[\rho(t)] \\
& t \geq 0
\end{aligned}
$$

where the last equality follows by taking the derivative inside the trace and noting that

$$
\frac{d}{d t} \rho(t)=-i \hat{H} \rho(t)+i \rho(t) \hat{H}^{\dagger} .
$$

The mean and standard deviation of the tick distribution is

$$
\mu:=\int_{0}^{\infty} t P(t) d t, \quad \underline{\sigma}:=\sqrt{\int_{0}^{\infty}(t-\mu)^{2} P(t) d t} .
$$


Since this example of a quantum clock is a reset clock, we are only interested in the accuracy of the first tick, $R_{1}$ since the accuracy of later ticks is determined solely from it; $R_{j}=j R_{1}$ (see Sec. II E, or Appendix B 1 a, and Remark 6 for a detailed argument). Namely, we are interested in bounding the quantity

$$
R_{1}:=\frac{\mu^{2}}{\underline{\sigma}^{2}}
$$

\section{Overview of the quasi-ideal clock}

In this section, we recall some of the definitions and the core theorem from Ref. [8], which we need in this Appendix. This section sets some of the terminology and definitions needed for the proof of Theorem 6 .

\section{a. The generator of dynamics and potential function $\overline{\boldsymbol{V}}_{\mathbf{0}}$}

We start by introducing a generator of dynamics for the quasi-ideal clock.

$$
\hat{H}=\hat{V}_{d}+\hat{H}_{C} .
$$

The clock's free Hamiltonian, $\hat{H}_{C}$ is a truncated harmonic oscillator Hamiltonian. Namely, $\hat{H}_{C}=\sum_{n=0}^{d-1} \omega n|n\rangle\langle n|$. The free evolution of any initial clock state under this Hamiltonian has a period of $T_{0}=2 \pi / \omega$, specifically, $e^{-i T_{0} \hat{H}_{C}} \rho_{C} e^{i T_{0} \hat{H}_{C}}=\rho_{C}$ for all $\rho_{C}$. The clock interaction term $\hat{V}_{d}$, takes the form

$$
\hat{V}_{d}=\frac{d}{T_{0}} \sum_{k=0}^{d-1} V_{d}(k)\left|\theta_{k}\right\rangle\left\langle\theta_{k}\right|,
$$

where the basis $\left\{\left|\theta_{k}\right\rangle\right\}_{k=0}^{d-1}$ is the Fourier transform of the energy eigenbasis $\{|n\rangle\}_{n=0}^{d-1}$, specifically,

$$
\left|\theta_{k}\right\rangle=\frac{1}{\sqrt{d}} \sum_{n=0}^{d-1} e^{-i 2 \pi n k / d}\left|E_{n}\right\rangle .
$$

In other words, we choose $\left\{\left|\theta_{k}\right\rangle\right\}$ to be the time basis. It will also be useful later to have the range of $k$ extended to $\mathbb{Z}$ [54]. Extending the range of $k$ in Eq. (F9) it follows that $\left|\theta_{k}\right\rangle=\left|\theta_{k \text { mod. } d}\right\rangle$ for $k \in \mathbb{Z}$. It is this basis that we identify with the time basis, i.e., we set $\left|\theta_{k}\right\rangle=\left|t_{k}\right\rangle$, $k \in \mathbb{N}_{\geq 0}$. The function $V_{d}: \mathbb{R} \mapsto \mathbb{R} \cup \mathbb{H}^{-}$(where $\mathbb{H}^{-}:=$ $\left\{a_{0}+i b_{0}: a_{0} \in \mathbb{R}, b_{0}<0\right\}$ denotes the lower-half complex plane) is defined by

$$
V_{d}(x)=\frac{2 \pi}{d} V_{0}\left(\frac{2 \pi}{d} x\right),
$$

where $V_{0}$ is any infinitely differentiable periodic function of period $2 \pi$. We are only interested in a specialized case, and from here on choose $V_{d}$ to map $\mathbb{R} \mapsto-i \mathbb{R}_{\geq 0}$. As one might imagine, it will be more convenient to work with a real function. We also want to make explicit the normalization. We thus write the potential function in terms of an explicitly positive, normalized function by defining $\bar{V}_{d}: \mathbb{R} \rightarrow \mathbb{R}_{>0}$ and $\bar{V}_{0}: \mathbb{R} \rightarrow \mathbb{R}_{>0}$,

$$
\begin{aligned}
-i \delta \bar{V}_{d}(x):=V_{d}(x), \\
-i \delta \bar{V}_{0}(x):=V_{0}(x), \quad \int_{0}^{2 \pi} d x \bar{V}_{0}(x)=1, \quad \bar{V}_{0}(x)>0 \\
\forall x \in \mathbb{R},
\end{aligned}
$$

where we specialize to the case $\delta \geq 1$. The last constraint ensures that $\bar{V}_{0}$ has full support. Secondly, it will be useful to let $\bar{V}_{0}$ have a unique global maximum in the interval $x \in[0,2 \pi]$ at $x=x_{0}$. Let the parameters $\tilde{\epsilon}_{V}, x_{v l}, x_{x r}$ be such that

$$
1-\tilde{\epsilon}_{V}=\int_{x_{v l}}^{x_{v r}} d x \bar{V}_{0}\left(x+x_{0}\right)
$$

for some $-\pi \leq x_{v l}<0<x_{v r} \leq \pi$, where, for simplicity, we set $x_{v l}=-x_{v r}$. Furthermore, we find that it is convenient to set $x_{0}=\pi+x_{v r}+\pi \gamma$. These are all the properties of $\bar{V}_{0}$, which we need for the lemmas in Secs. 3-6. In Sec. 7, we find explicit parametrizations of $x_{v r}$ and $\gamma$ in terms of $d$.

\section{b. Explicit form on the potential $\overline{\boldsymbol{V}}_{\mathbf{0}}$}

In Sec. F 7 we need to introduce an explicit function for the potential $\bar{V}_{0}$ satisfying the criteria from Sec. F 2 a. The function is written in terms of parameters $\delta \geq 1, n \geq$ $1, N \in \mathbb{N}_{>0}$. Specific values for these parameters is chosen in Sec. F 7 in order to satisfy specific conditions. Here we summarize the final form of the potential $\bar{V}_{0}$ to which Theorem 6 applies.

For $d \geq 3$, let

$$
\bar{V}_{0}(x):=\frac{1}{\delta d^{2}}+n A_{0} \sum_{p=-\infty}^{\infty} V_{B}\left[n\left(x-x_{0}-2 \pi p\right)\right],
$$

where

$$
A_{0}=\frac{1-2 \pi /\left(\delta d^{2}\right)}{\int_{-\infty}^{\infty} d x V_{B}(x)}
$$

is a normalization parameter chosen such that

$$
\int_{0}^{2 \pi} \bar{V}_{0}(x) d x=1,
$$

and

$$
V_{B}(x):=\operatorname{sinc}^{2 N}(x):=\left(\frac{\sin (\pi x)}{\pi x}\right)^{2 N},
$$


where $V_{B}(0)=1$ and $N \in \mathbb{N}_{>0} . N$ is given by

$$
N=\left\lceil\frac{3-4 \epsilon_{5}-\epsilon_{9}}{2\left(\epsilon_{7}-\epsilon_{8}-\epsilon_{5}\right)}\right\rceil,
$$

with $\lceil\cdot\rceil$ the ceiling function, and where $\epsilon_{7}=\eta / 4, \epsilon_{5}=$ $\epsilon_{8}=\eta / 16$, and $\epsilon_{9}=\eta / 2$, and the role of $\eta \in(0,1)$ becomes apparent in Theorem 36. The parameter $\delta$ is given by

$$
\delta=d^{\epsilon 5} .
$$

Let $C_{0}: \mathbb{N}_{>0} \mapsto \mathbb{R}_{>0}$ be any function, which is only a function of $N$ (i.e., independent of $n, d$, and $k$ ), such that

$$
\max _{x \in[0,2 \pi]}\left|\frac{d^{k}}{d x^{k}} \bar{V}_{0}(x)\right| \leq n^{k+1} C_{0}^{k+1}, \quad \forall k \in \mathbb{N}_{\geq 0}, \forall n \geq 1,
$$

holds. We prove later by explicit construction that such functions exist. We now define $n$ to be

$$
n=\frac{\ln \left(\pi \alpha_{0} \sigma^{2}\right)}{2 \pi C_{0} \alpha_{0} \kappa} \frac{d^{1-\epsilon_{5}}}{\delta \sigma},
$$

where $\kappa=0.792$ and $\alpha_{0} \in(0,1]$ depends on the mean energy of the initial quasi-ideal clock state and is defined in Sec. F 2 c. Finally, the location of the peak of the potential, $x_{0}$, is given by $x_{0}=\pi+x_{v r}+\pi \gamma$, where

$$
\begin{gathered}
x_{v r}=d^{\epsilon 7} \frac{\sigma}{\pi d}, \\
\gamma=\frac{m-2}{d},
\end{gathered}
$$

where

$$
\begin{aligned}
& m= \begin{cases}2\lfloor\bar{m}\rfloor & \text { if } d=2,4,6, \ldots, \\
2\lfloor\bar{m}\rfloor+1 & \text { if } d=3,5,7, \ldots,\end{cases} \\
& \bar{m}= \begin{cases}\frac{d^{\eta / 2}}{2} \sigma+1 & \text { if } d=2,4,6, \ldots, \\
\frac{d^{\eta / 2}}{2} \sigma+\frac{1}{2} & \text { if } d=3,5,7, \ldots\end{cases}
\end{aligned}
$$

With the above definitions, one can write the function $\bar{V}_{0}(x)$ solely in terms of parameters $d, \eta, \sigma$, and $\alpha_{0}$.

\section{c. The quasi-ideal clock states}

Recall that for the quasi-ideal clock, the initial state is pure $\rho_{C}=\left|\Psi_{\text {nor }}\left(k_{0}\right)\right\rangle\left\langle\Psi_{\text {nor }}\left(k_{0}\right)\right|$, where

$$
\begin{gathered}
\left|\Psi_{\text {nor }}\left(k_{0}\right)\right\rangle=\sum_{k \in \mathcal{S}_{d}\left(k_{0}\right)} \psi_{\text {nor }}\left(k_{0} ; k\right)\left|\theta_{k}\right\rangle, \\
\psi_{\text {nor }}\left(k_{0} ; x\right)=A e^{-\frac{\pi}{\sigma^{2}}\left(x-k_{0}\right)^{2}} e^{i 2 \pi n_{0}\left(x-k_{0}\right) / d}, \quad x \in \mathbb{R},
\end{gathered}
$$

with $\sigma \in(0, d), n_{0} \in(0, d-1), k_{0} \in \mathbb{R}, A \in \mathbb{R}^{+}$, and $\mathcal{S}_{d}\left(k_{0}\right)$ is the set of $d$ integers closest to $k_{0}$, defined as

$$
\mathcal{S}_{d}\left(k_{0}\right)=\left\{k: k \in \mathbb{Z} \text { and }-\frac{d}{2} \leq k_{0}-k<\frac{d}{2}\right\} .
$$

$A$ is defined so that the state is normalized, namely

$$
A=A\left(\sigma ; k_{0}\right)=\frac{1}{\sqrt{\sum_{k \in \mathcal{S}_{d}\left(k_{0}\right)} e^{-\frac{2 \pi}{\sigma^{2}}\left(k-k_{0}\right)^{2}}}},
$$

which is of order $A=\mathcal{O}\left(\left(2 / \sigma^{2}\right)^{1 / 4}\right)$ as $d \rightarrow \infty$ for all $k_{0} \in \mathbb{R} . \omega n_{0}$ is approximately the mean energy of the initial clock state. We use $n_{0}$ to define $\alpha_{0} \in(0,1]$. It is defined in Definition 1 in Ref. [8] as

$$
\begin{aligned}
\alpha_{0} & =\left(\frac{2}{d-1}\right) \min \left\{n_{0},(d-1)-n_{0}\right\} \\
& =1-\left|1-n_{0}\left(\frac{2}{d-1}\right)\right| \in(0,1] .
\end{aligned}
$$

Physically, it is a measure of the distance of the mean energy from the edge of the energy spectrum, and has its maximum value $\alpha=1$ when the the mean energy is in the midpoint of the spectrum, namely when $n_{0}=(d-1) / 2$. We pick $\alpha_{0}$ to be a fixed constant throughout this paper. This means that $n_{0}$ takes on the value $n_{0}=\tilde{n}_{0}(d-1)$ with $\tilde{n}_{0} \in(0,1)$ fixed constant.

In the proof of Theorem 2, we need the core theorem in Ref. [8] (Theorem IX.1, p. 35). For brevity, we state it in a slightly reduced form, which is nevertheless adequate for our purposes. Intuitively, the theorem states that the evolution of quasi-ideal clock states under the generator $\hat{H}$ [Eq. (F7)] mimics the evolution of the idealized clock to a good approximation. In order to state it, we need to recall some definitions from Ref. [8]. Namely Definitions 8 and 9 on p. 21 [8].

Let $b$ be any real number satisfying

$$
b \geq \sup _{k \in \mathbb{N}^{+}}\left(2 \max _{x \in[0,2 \pi]}\left|V_{0}^{(k-1)}(x)\right|\right)^{1 / k},
$$

where $V_{0}^{(p)}(x)$ is the $p^{\text {th }}$ derivative with respect to $x$ of $V_{0}(x)$ and $V_{0}^{(0)}:=V_{0}$. We can use $b$ to define $\mathcal{N} \in \mathbb{N}^{0}$ as follows:

$$
\mathcal{N}=\left\lfloor\frac{\pi \alpha_{0}^{2}}{2\left(\bar{v}+\frac{d}{\sigma^{2}}\right)^{2}}\left(\frac{d}{\sigma}\right)^{2}\right\rfloor,
$$

where $\kappa=0.792$ and

$$
\bar{v}=\frac{\pi \alpha_{0} \kappa}{\ln \left(\pi \alpha_{0} \sigma^{2}\right)} b .
$$


The theorem states that for all $t \geq 0, k_{0} \in \mathbb{R}$,

$$
\begin{aligned}
e^{-i t\left(\hat{V}_{d}+\hat{H}_{C}\right)}\left|\Psi_{\text {nor }}\left(k_{0}\right)\right\rangle & =\left|\bar{\Psi}_{\text {nor }}\left(k_{0}+t d / T_{0}, t d / T_{0}\right)\right\rangle+\left|\varepsilon_{v}\right\rangle \\
& =\sum_{k \in \mathcal{S}_{d}\left(k_{0}+t d / T_{0}\right)} e^{-i \int_{k-t d / T_{0}}^{k} d y V_{d}(y)} \psi_{\text {nor }}\left(k_{0}+t d / T_{0} ; k\right)\left|\theta_{k}\right\rangle+\left|\varepsilon_{v}\right\rangle,
\end{aligned}
$$

where $\left|\varepsilon_{v}\right\rangle=\left|\varepsilon_{v}\right\rangle(t, d)$. The important question is how small can the error term be, namely how does

$$
\varepsilon_{v}(t, d):=\|\left|\varepsilon_{v}\right\rangle(t, d) \|_{2}
$$

scale with the properties of the quasi-ideal clock and generator? The theorem puts bounds on this scaling. Namely if

$$
\bar{v} \geq 0, \quad \mathcal{N} \geq 8,
$$

are both satisfied, the theorem tells us that in the limits $d \rightarrow \infty,(0, d) \ni \sigma \rightarrow \infty$,

$$
\begin{aligned}
\varepsilon_{v}(t, d)= & |t| \frac{d}{T_{0}}\left[\mathcal{O}\left(\frac{\sigma^{3}}{\bar{v} \sigma^{2} / d+1}\right)^{1 / 2}+\mathcal{O}\left(\frac{d^{2}}{\sigma^{2}}\right)\right] \exp \left[-\frac{\pi}{4} \frac{\alpha_{0}^{2}}{\left(\frac{d}{\sigma^{2}}+\bar{v}\right)^{2}}\left(\frac{d}{\sigma}\right)^{2}\right] \\
& +\mathcal{O}\left(|t| \frac{d^{2}}{\sigma^{2}}+1\right) e^{-\frac{\pi}{4} \frac{d^{2}}{\sigma^{2}}}+\mathcal{O}\left(e^{-\frac{\pi}{2} \sigma^{2}}\right)
\end{aligned}
$$

\section{d. An expression for $\boldsymbol{R}_{\mathbf{1}}$ for the quasi-ideal clock}

In this section, we show how to express $\operatorname{tr}[\rho(t)]$ from Sec. F 1 in terms of the quasi-ideal clock from Ref. [8] discussed in Secs. F 2 a and F 2 c. Quasi-ideal clock, namely $\rho_{0}=\left|\psi_{0}\right\rangle\left\langle\psi_{0}|=| \psi_{\text {nor }}\left(k_{0}\right)\right\rangle\left\langle\psi_{\text {nor }}\left(k_{0}\right)\right|$. From Eq. (F35) it follows

$$
\begin{aligned}
\operatorname{tr}[\rho(t)] & =\sum_{k \in \mathcal{S}_{d}\left(k_{0}+t d / T_{0}\right)}\left|\left\langle\theta_{k}\left|e^{-i t \hat{H}}\right| \psi_{\text {nor }}\left(k_{0}\right)\right\rangle\right|^{2} \\
& =\sum_{k \in \mathcal{S}_{d}\left(k_{0}+t d / T_{0}\right)}\left|e^{-i \int_{k-t d / T_{0}}^{k} d y V_{d}(y)} \psi_{\text {nor }}\left(k_{0} ; k-t d / T_{0}\right)+\left\langle\theta_{k} \mid \varepsilon_{\nu}\right\rangle(t, d)\right|^{2},
\end{aligned}
$$

where we use that $\psi_{\text {nor }}\left(k_{0}+t d / T_{0} ; k\right)=\psi_{\text {nor }}\left(k_{0} ; k-t d / T_{0}\right)$, which follows directly from Eq. (F26). Due to the normalization of $\left|\psi_{\text {nor }}\left(k_{0}\right)\right\rangle$, we have

$$
\sum_{k \in \mathcal{S}_{d}\left(k_{0}+t d / T_{0}\right)}\left|\psi_{\text {nor }}\left(k_{0} ; k-t d / T_{0}\right)\right|^{2}=1
$$

\section{Approximating $\boldsymbol{R}_{1}$}

We now proceed to lower bound $R_{1}$ in terms of dimension and mean clock energy for the quasi-ideal clock. Specifically in this section, up to additive error terms, the aim will be to express $R_{1}$ in terms of the first and second moments of $\operatorname{tr}[\rho(t)]$ with integration range limited to one period of the free clock Hamiltonian, namely to the interval $\left[0, T_{0}\right]$.

\section{a. A more useful expression for $\boldsymbol{R}_{1}$}

Lemma 19: Let $\Delta(0)$ and $\Delta(1)$ satisfy

$$
\Delta^{2}(0) \leq\left(\int_{0}^{T_{0}} d t \operatorname{tr}[\rho(t)]\right)^{2}, \quad \Delta(1) \geq \int_{0}^{T_{0}} d t \operatorname{tr}[\rho(t)] \geq 0 .
$$

then

$$
R_{1} \geq \frac{\Delta^{2}(0)+\epsilon_{2}}{2 \Delta(1)-\Delta^{2}(0)+\epsilon_{1}}
$$


where the epsilon terms $\epsilon_{2}, \epsilon_{1}$ are defined by

$$
\epsilon_{2}:=2 \mu_{0} \mu_{\epsilon}+\mu_{\epsilon}^{2}
$$

with

$$
\begin{aligned}
& \mu_{0}:=\int_{0}^{T_{0}} d t \operatorname{tr}[\rho(t)], \\
& \mu_{\epsilon}:=-\lim _{t \rightarrow \infty} t \operatorname{tr}[\rho(t)]+\int_{T_{0}}^{\infty} d t \operatorname{tr}[\rho(t)],
\end{aligned}
$$

and

$$
\epsilon_{1}:=-2 \mu_{0} \mu_{\epsilon}-\mu_{\epsilon}^{2}+2 \int_{T_{0}}^{\infty} d t \operatorname{tr}[\rho(t)]-\lim _{t \rightarrow \infty}\left[2 \mu t+(t-\mu)^{2}\right] \operatorname{tr}[\rho(t)] .
$$

Proof. By integration by parts,

$$
\begin{aligned}
\mu & =\int_{0}^{\infty} t P(t) d t=-\left.\{t \operatorname{tr}[\rho(t)]\}\right|_{0} ^{\infty}+\int_{0}^{\infty} d t \operatorname{tr}[\rho(t)] \\
& =\mu_{0}+\mu_{\epsilon} .
\end{aligned}
$$

Similarly,

$$
\begin{aligned}
\underline{\sigma}^{2} & =\int_{0}^{\infty}(t-\mu)^{2} P(t) d t=-\left.\left\{(t-\mu)^{2} \operatorname{tr}[\rho(t)]\right\}\right|_{0} ^{\infty}+2 \int_{0}^{\infty} d t(t-\mu) \operatorname{tr}[\rho(t)] \\
& =-\lim _{t \rightarrow \infty}(t-\mu)^{2} \operatorname{tr}[\rho(t)]+\mu^{2}+2 \int_{0}^{\infty} d t t \operatorname{tr}[\rho(t)]-2 \mu\left\{\mu+\lim _{t \rightarrow \infty} t \operatorname{tr}[\rho(t)]\right\} \\
& =-\mu^{2}+2 \int_{0}^{\infty} d t t \operatorname{tr}[\rho(t)]-\lim _{t \rightarrow \infty}\left[2 \mu t+(t-\mu)^{2}\right] \operatorname{tr}[\rho(t)] \\
& =-\mu_{0}^{2}+2 \int_{0}^{T_{0}} d t t \operatorname{tr}[\rho(t)]+\epsilon_{1} \leq-\mu_{0}^{2}+2 \Delta(1)+\epsilon_{1} \\
& \leq-\Delta^{2}(0)+2 \Delta(1)+\epsilon_{1}
\end{aligned}
$$

From Eqs. (F54) and (F49), we find

$$
\begin{aligned}
R_{1} & =\frac{\mu^{2}}{\underline{\sigma}^{2}} \geq \frac{\mu_{0}^{2}+2 \mu_{0} \mu_{\epsilon}+\mu_{\epsilon}^{2}}{-\Delta^{2}(0)+2 \Delta(1)+\epsilon_{1}} \\
& \geq \frac{\Delta^{2}(0)+2 \mu_{0} \mu_{\epsilon}+\mu_{\epsilon}^{2}}{-\Delta^{2}(0)+2 \Delta(1)+\epsilon_{1}} .
\end{aligned}
$$

We now bound $\epsilon_{1}$ and $\epsilon_{2}$ appearing in Lemma 19. These terms are negligible in the large $d$ limit and originate from the tails of the integrals in the definition of $\mu$ and $\underline{\sigma}$. 
Lemma 20: $\epsilon_{1}$ and $\epsilon_{2}$, defined in Eqs. (F47) and (F44), respectively, are bounded by

$$
\begin{aligned}
\left|\epsilon_{1}\right| & \leq\left\{a^{2}\left[e^{-\delta}+\varepsilon_{v}\left(T_{0}, d\right)\right]^{2}+2\left(2 a T_{0}+a^{2}\right)\right\}\left[e^{-\delta}+\varepsilon_{v}\left(T_{0}, d\right)\right]^{2}, \\
0 \leq \epsilon_{2} & \leq\left\{2 T_{0} a+a^{2}\left[e^{-\delta}+\varepsilon_{v}\left(T_{0}, d\right)\right]^{2}\right\}\left[e^{-\delta}+\varepsilon_{v}\left(T_{0}, d\right)\right]^{2},
\end{aligned}
$$

where $a$ is any parameter satisfying

$$
a \geq \frac{T_{0}}{4 \pi \delta}\left(\min _{x \in[0,2 \pi]} \bar{V}_{0}(x)\right)^{-1}
$$

and $\varepsilon_{v}\left(T_{0}, d\right)$ is defined by Eq. (F36). Note that since we have chosen $\bar{V}_{0}$ to have full support, a is finite.

Proof. We start by bounding $\operatorname{tr}[\rho(t)]$. We find that

$$
\operatorname{tr}[\rho(t)]=\operatorname{tr}\left[e^{-i t \hat{H}} \rho_{0} e^{i t \hat{H}^{\dagger}}\right] \leq\left\|e^{-i t \hat{H}} e^{i t \hat{H}^{\dagger}}\right\|_{\infty} \leq\left\|e^{-i t \hat{H}}\right\|_{\infty}\left\|e^{i t \hat{H}^{\dagger}}\right\|_{\infty}
$$

where $\|\cdot\|_{\infty}$ is the Schatten $p=\infty$ norm also called the operator norm. Recalling $\hat{H}=\hat{H}_{C}-i \hat{V}_{C}$, and applying the Golden-Thompson inequality, which holds for the operator norm [55], we find that

$$
\operatorname{tr}[\rho(t)] \leq\left\|e^{-i t \hat{H}_{C}}\right\|_{\infty}\left\|e^{i t \hat{H}_{C}}\right\|_{\infty}\left\|e^{-t \hat{V}_{C}}\right\|_{\infty}^{2}=\left\|e^{-t \hat{V}_{C}}\right\|_{\infty}^{2}=\exp \left\{-\delta t \frac{4 \pi}{T_{0}}\left[\min _{k \in\{0,1,2, \ldots, d-1\}} \bar{V}_{0}\left(\frac{2 \pi}{d} k\right)\right]\right\}
$$

where we use $\hat{V}_{C}=i \hat{V}_{d}:=\delta d / T_{0} \sum_{k=0}^{d-1} \bar{V}_{d}(k)\left|\theta_{k}\right\rangle\left\langle\theta_{k}\right|$, where $d / T_{0} \bar{V}_{d}(k)=2 \pi / T_{0} \bar{V}_{0}[(2 \pi / d) k]$. Now, in this paper, $\bar{V}_{0}$ is positive [Eq. (F12)]. As such, for all $d \in \mathbb{N}_{>0}$

$$
\min _{k \in\{0,1,2, \ldots, d-1\}} \bar{V}_{0}\left(\frac{2 \pi}{d} k\right)>0
$$

and thus

$$
\lim _{t \rightarrow \infty} t^{n} \operatorname{tr}[\rho(t)]=0
$$

for all $n \geq 0$ and $d \in \mathbb{N}_{>0}$. Our next task is to bound the two integrals $\int_{T_{0}}^{\infty} d t \operatorname{tr}[\rho(t)]$ and $\int_{T_{0}}^{\infty} d t t \operatorname{tr}[\rho(t)]$. We $\operatorname{start}$ by finding a $t$ independent parameter $a>0$ such that $\operatorname{tr}[\rho(t)] \leq a P(t)$ for all $t \geq 0$, where $P(t)=-(d / d t) \operatorname{tr}[\rho(t)]$ was defined in Eq. (F3):

$$
\begin{aligned}
\frac{\operatorname{tr}[\rho(t)]}{P(t)} & =\frac{\operatorname{tr}[\rho(t)]}{i \operatorname{tr}[\hat{H} \rho(t)]-i \operatorname{tr}\left[\hat{H}^{\dagger} \rho(t)\right]}=\frac{\operatorname{tr}[\rho(t)]}{i \operatorname{tr}\left[\left(\hat{H}_{C}-i \hat{V}_{C}\right) \rho(t)\right]-i \operatorname{tr}\left[\left(\hat{H}_{C}+i \hat{V}_{C}\right) \rho(t)\right]}=\frac{1}{2 \operatorname{tr}\left[\hat{V}_{C} \bar{\rho}(t)\right]} \\
& \leq \frac{1}{2}\left(\inf _{|\psi\rangle \in \mathcal{S}_{p}} \operatorname{tr}\left[\hat{V}_{C}|\psi\rangle\langle\psi|\right]\right)^{-1}, \quad \forall t \geq 0,
\end{aligned}
$$

where $\bar{\rho}(t):=\rho(t) / \operatorname{tr}[\rho(t)]$ is a normalized rank-1 density matrix for all $t \geq 0$ and $\mathcal{S}_{p}$ is the set of normalized pure quantum states (rank-1 density matrices). Crucially, note that $P(t)>0$ since $\operatorname{tr}\left[\hat{V}_{C} \bar{\rho}(t)\right]>0$ because $\hat{V}_{C}$ is positive definite 
since $\bar{V}_{0}$ has full support. Hence taking the trace in the orthonormal basis $\left\{\left|\theta_{k}\right\rangle\right\}_{k=0}^{d-1}$,

$$
\begin{aligned}
\frac{\operatorname{tr}[\rho(t)]}{P(t)} & \leq \frac{1}{2}\left(\min _{k \in\{0,1,2, \ldots, d-1\}}\left\langle\theta_{k}\left|\hat{V}_{C}\right| \theta_{k}\right\rangle\right)^{-1}=\frac{T_{0}}{4 \pi \delta}\left(\min _{k \in\{0,1,2, \ldots, d-1\}} \bar{V}_{0}(2 \pi k / d)\right)^{-1} \\
& \leq \frac{T_{0}}{4 \pi \delta}\left(\min _{x \in[0,2 \pi]} \bar{V}_{0}(x)\right)^{-1} \leq a, \quad \forall t \geq 0
\end{aligned}
$$

Using Eq. (F67) can now bound the first integral:

$$
\int_{T_{0}}^{\infty} d t \operatorname{tr}[\rho(t)] \leq a \int_{T_{0}}^{\infty} d t P(t)=-a[\operatorname{tr}[\rho(t)]]_{T_{0}}^{\infty}=a\left(\operatorname{tr}\left[\rho\left(T_{0}\right)\right]-\lim _{t \rightarrow \infty} \operatorname{tr}[\rho(t)]\right)=a \operatorname{tr}\left[\rho\left(T_{0}\right)\right] .
$$

For the second integral, we additionally have to integrate by parts and recall $T_{0}>0$ :

$$
\begin{gathered}
\int_{T_{0}}^{\infty} d t t \operatorname{tr}[\rho(t)] \leq-a \int_{T_{0}}^{\infty} d t t \frac{d}{d t} \operatorname{tr}[\rho(t)]=-a\left([t \operatorname{tr}[\rho(t)]]_{T_{0}}^{\infty}-\int_{T_{0}}^{\infty} d t \operatorname{tr}[\rho(t)]\right)=a T_{0} \operatorname{tr}\left[\rho\left(T_{0}\right)\right]+a \int_{T_{0}}^{\infty} d t \operatorname{tr}[\rho(t)] \\
\leq\left(a T_{0}+a^{2}\right) \operatorname{tr}\left[\rho\left(T_{0}\right)\right]
\end{gathered}
$$

where to achieve the last line, we use Eq. (F68). In order for Eqs. (F68) and (F69) to be useful, we need to bound $\operatorname{tr}\left[\rho\left(T_{0}\right)\right]$. This can be achieved using Eq. (F39). One finds that

$$
\operatorname{tr}\left[\rho\left(T_{0}\right)\right]=\left|e^{-\delta}+\left\langle\theta_{k} \mid \varepsilon_{v}\right\rangle\left(T_{0}, d\right)\right|^{2} \leq\left(e^{-\delta}+\varepsilon_{v}\left(T_{0}, d\right)\right)^{2} .
$$

Finally, recalling that $-(d / d t) \operatorname{tr}[\rho(t)]>0$, we have

$$
\mu_{0}=\int_{0}^{T_{0}} d t \operatorname{tr}[\rho(t)] \leq T_{0} \max _{t \in\left[0, T_{0}\right]} \operatorname{tr}[\rho(t)]=T_{0} \operatorname{tr}[\rho(0)]=T_{0} .
$$

Thus using Eqs. (F63), (F68), (F69), (F71) and (F72), it follows that $\epsilon_{1}$ defined in Eq. (F47) is bounded by

$$
\left|\epsilon_{1}\right| \leq 2 T_{0} \mu_{\epsilon}+\mu_{\epsilon}^{2}+2\left(a T_{0}+a^{2}\right)\left[e^{-\delta}+\varepsilon_{v}\left(T_{0}, d\right)\right]^{2} .
$$

Similarly, we find that $\mu_{\epsilon}$ defined in Eq. (F45) is bounded by

$$
\mu_{\epsilon} \leq a\left[e^{-\delta}+\varepsilon_{v}\left(T_{0}, d\right)\right]^{2} .
$$

Thus using the definition of $\epsilon_{2}$ in Eq. (F44), we complete the proof.

\section{Upper and lower bounds for $\operatorname{tr}[\rho(t)]$}

\section{Lemma 21:}

$$
\left(\sum_{k \in \mathcal{S}_{d}\left(k_{0}+t d / T_{0}\right)} \Delta_{k}\right)-\epsilon_{0} \leq \operatorname{tr}[\rho(t)] \leq\left(\sum_{k \in \mathcal{S}_{d}\left(k_{0}+t d / T_{0}\right)} \Delta_{k}\right)+\epsilon_{0}
$$

where

$$
\Delta_{k}:=e^{-2 \delta \int_{k-t d / T_{0}}^{k} d y \bar{V}_{d}(y)}\left|\psi_{n o r}\left(k_{0} ; k-t d / T_{0}\right)\right|^{2}, \quad \epsilon_{0}=\epsilon_{0}(t, d):=\varepsilon_{v}(t, d)\left[\varepsilon_{v}(t, d)+2\right] d,
$$

and $\varepsilon_{v}(t, d)$ is defined by Eq. (F36). 
Proof. Here we use the core theorem in Ref. [8] discussed in Sec. d. Using Eqs. (F39) and (F75) follows from noting $|a+\epsilon|^{2}=|a|^{2}+|\epsilon|^{2}+2 \mathfrak{R}(a \epsilon), \mathfrak{R}(a) \leq|a|$ for $a, \epsilon \in \mathbb{C}$, and $\left|\left\langle\theta_{k} \mid \varepsilon_{v}\right\rangle(t, d)\right| \leq \varepsilon_{v}(t, d)$ for all $k$.

We now calculate time-dependent upper and lower bounds for $\sum_{k \in \mathcal{S}_{d}\left(k_{0}+t d / T_{0}\right)} \Delta_{k}$, where $\Delta_{k}$ is defined in Lemma 21 .

Lemma 22: Let $k_{0}=0$. There exists $\Delta_{L} \geq 0, \Delta_{R} \geq 0, \Delta_{C} \geq 0$ such that

$$
\sum_{k \in \mathcal{S}_{d}\left(k_{0}+t d / T_{0}\right)} \Delta_{k}=\Delta_{L}+\Delta_{C}+\Delta_{R}
$$

where $\Delta_{L}, \Delta_{R}$ satisfy the bounds

$$
\Delta_{L}, \Delta_{R} \leq A^{2} \frac{e^{-\frac{2 \pi}{\sigma^{2}}[\gamma d / 2-\bar{k}(t)]^{2}}}{1-e^{-4 \pi|\gamma d / 2-\bar{k}(t)| / \sigma^{2}}}
$$

where $\bar{k}(t):=\left\lfloor-d / 2+t d / T_{0}+1\right\rfloor+d / 2-t d / T_{0} \in[0,1]$ and

$$
\gamma=\gamma(m):=\frac{m-2}{d} \in(0,1), \quad m=\left\{\begin{array}{l}
4,6,8 \ldots, d+2 \text { if } d=2,4,6, \ldots \\
3,5,7, \ldots, d+2 \text { if } d=3,5,7, \ldots
\end{array}\right.
$$

Furthermore, there exists $\Delta_{C}^{(-)} \geq 0, \Delta_{C}^{(+)} \geq 0$, such that $\Delta_{C}$ satisfies the bounds

$$
\Delta_{C}^{(-)}-\epsilon_{C} \leq \Delta_{C} \leq \Delta_{C}^{(+)}
$$

where

$$
\epsilon_{C}=\epsilon_{C}(t):=2 A^{2} \frac{e^{-\frac{2 \pi}{\sigma^{2}}[\gamma d / 2-\bar{k}(t)]^{2}}}{1-e^{-4 \pi|\gamma d / 2-\bar{k}(t)| / \sigma^{2}}}
$$

and if $x_{0}$ (defined in Sec. a) satisfies $x_{v r}+\pi \gamma \leq x_{0} \leq 2 \pi-x_{v r}-\pi \gamma$, then

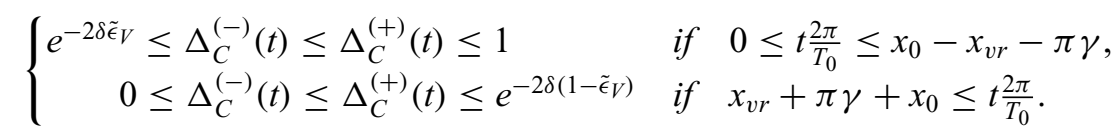

When the value of $t$ is such that the above bounds do not hold, we can also use the bounds

$$
0 \leq \Delta_{C}(t) \leq 1, \quad \forall t \geq 0, x_{0} \in \mathbb{R}
$$

Proof.

$$
\begin{aligned}
\sum_{k \in \mathcal{S}_{d}\left(k_{0}+t d / T_{0}\right)} \Delta_{k} & =\sum_{\substack{k \in \mathcal{S}_{d}\left(k_{0}+t d / T_{0}\right) \\
\max \left\{\mathcal{S}_{d}\left(k_{0}+t d / T_{0}\right)\right\}-t d / T_{0}}} e^{-2 \delta \int_{k-t d / T_{0}}^{k} d y \bar{V}_{d}(y)}\left|\psi_{\text {nor }}\left(k_{0} ; k-t d / T_{0}\right)\right|^{2} \\
= & \sum_{k=\min \left\{\mathcal{S}_{d}\left(k_{0}+t d / T_{0}\right)\right\}-t d / T_{0}}^{-2 \delta \int_{k}^{k+t d / T_{0}} d y \bar{V}_{d}(y)}\left|\psi_{\text {nor }}\left(k_{0} ; k\right)\right|^{2} \\
= & \sum_{k=\left\lfloor-d / 2+k_{0}+1+t d / T_{0}\right\rfloor-t d / T_{0}}^{\left\lfloor d / 2+k_{0}+t d / T_{0}\right\rfloor-t d / T_{0}} e^{-2 \delta \int_{k}^{k+t d / T_{0}} d y \bar{V}_{d}(y)}\left|\psi_{\text {nor }}\left(k_{0} ; k\right)\right|^{2} \\
= & \sum_{k=k_{0}-d / 2+d / 2+\bar{k}(t)-1} e^{-2 \delta \int_{k}^{k+t d / T_{0}} d y \bar{V}_{d}(y)}\left|\psi_{\text {nor }}\left(k_{0} ; k\right)\right|^{2},
\end{aligned}
$$

where recall $\bar{k}(t)=\left\lfloor-d / 2+k_{0}+t d / T_{0}+1\right\rfloor+d / 2-k_{0}-t d / T_{0} \in[0,1]$. This follows from noting that $\bar{k}(t)$ is a solution to both equations $\left\lfloor-d / 2+k_{0}+1+t d / T_{0}\right\rfloor-t d / T_{0}=k_{0}-d / 2+\bar{k}(t)$ and $\left\lfloor d / 2+k_{0}+t d / T_{0}\right\rfloor-t d / T_{0}=k_{0}+d / 2+$ 
$\bar{k}(t)-1$, since we can use the identity $1=\lfloor-x+y+1\rfloor-\lfloor x+y\rfloor+2 x$, for $2 x \in \mathbb{Z}, y \in \mathbb{R}$ and set $x=d / 2, y=k_{0}+$ $t d / T_{0}$. For simplicity, we now take into account that $k_{0}=0$. We now break the sum up into three contributions where the first two will correspond to the "Gaussian tails" to the "left" $\left(\Delta_{L}\right)$ and "right" $\left(\Delta_{R}\right)$ of $k=k_{0}=0$, and a "central term" $\left(\Delta_{C}\right)$ corresponding to the region $k \approx k_{0}=0$. Namely,

$$
\sum_{k \in \mathcal{S}_{d}\left(k_{0}+t d / T_{0}\right)} \Delta_{k}=\Delta_{L}+\Delta_{C}+\Delta_{R}
$$

We introduce $\gamma \in(0,1)$ and start with bounding $\Delta_{L}$ :

$$
\begin{aligned}
\Delta_{L} & :=\sum_{k=-d / 2+\bar{k}(t)}^{\bar{k}(t)-\gamma d / 2} e^{-2 \delta \int_{k}^{k+t d / T_{0}} d y \bar{V}_{d}(y)}\left|\psi_{\text {nor }}\left(k_{0} ; k\right)\right|^{2} \leq \sum_{k=-d / 2+\bar{k}(t)}^{\bar{k}(t)-\gamma d / 2}\left|\psi_{\mathrm{nor}}\left(k_{0} ; k\right)\right|^{2}=\sum_{y=\gamma d / 2-\bar{k}(t)}^{d / 2-\bar{k}(t)} A^{2} e^{-\frac{2 \pi}{\sigma^{2}}(-y)^{2}} \\
& \leq \sum_{y=\gamma d / 2-\bar{k}(t)}^{\infty} A^{2} e^{-\frac{2 \pi}{\sigma^{2}}(-y)^{2}} \leq A^{2} \frac{e^{-\frac{2 \pi}{\sigma^{2}}(\bar{k}(t)-\gamma d / 2)^{2}}}{1-e^{-4 \pi|\bar{k}(t)-\gamma d / 2| / \sigma^{2}}}
\end{aligned}
$$

where the summations are defined as

$$
\sum_{y=a}^{b} f(y)=f(a)+f(a+1)+\ldots+f(b)
$$

where $a, b \in \mathbb{R}, b-a \in \mathbb{Z}$ and the sum is defined to be zero if $b-a \leq 0$. We use this convention throughout. We constrain $\gamma$ appropriately later in Eq. (F96). In the last line of Eq. (F90) we use Lemma J.0.1, p. 59 from Ref. [8]. Similarly, for $\Delta_{R}$,

$$
\begin{aligned}
\Delta_{R} & :=\sum_{k=\bar{k}(t)+\gamma d / 2}^{\bar{k}(t)+d / 2} e^{-2 \delta \int_{k}^{k+t d / T_{0}} d y \bar{V}_{d}(y)}\left|\psi_{\mathrm{nor}}\left(k_{0} ; k\right)\right|^{2} \leq \sum_{k=\bar{k}(t)+\gamma d / 2}^{\bar{k}(t)+d / 2}\left|\psi_{\mathrm{nor}}\left(k_{0} ; k\right)\right|^{2} \leq \sum_{k=\bar{k}(t)+\gamma d / 2}^{\infty}\left|\psi_{\mathrm{nor}}\left(k_{0} ; k\right)\right|^{2} \\
& \leq \sum_{k=\bar{k}(t)-\gamma d / 2}^{\infty}\left|\psi_{\mathrm{nor}}\left(k_{0} ; k\right)\right|^{2} \leq A^{2} \frac{e^{-\frac{2 \pi}{\sigma^{2}}(\bar{k}(t)-\gamma d / 2)^{2}}}{1-e^{-4 \pi|\bar{k}(t)-\gamma d / 2| / \sigma^{2}}}=A^{2} \frac{e^{-\frac{2 \pi}{\sigma^{2}}[\gamma d / 2-\bar{k}(t)]^{2}}}{1-e^{-4 \pi|\gamma d / 2-\bar{k}(t)| / \sigma^{2}}}
\end{aligned}
$$

For $\Delta_{C}$, we have

$$
\begin{gathered}
\Delta_{C}:=\sum_{k=\bar{k}(t)-\gamma d / 2+1}^{\bar{k}(t)+\gamma d / 2-1} e^{-2 \delta \int_{k}^{k+t d / T_{0}} d y \bar{V}_{d}(y)}\left|\psi_{\text {nor }}\left(k_{0} ; k\right)\right|^{2} \\
\left\{\begin{array}{l}
\leq\left(\max _{y \in \mathcal{I}_{\gamma}}\left\{e^{-2 \delta \int_{y}^{y+t d / T_{0}} d x \bar{V}_{d}(x)}\right\}\right) \sum_{k=\bar{k}(t)-\gamma d / 2+1}^{\bar{k}(t)+\gamma d / 2-1}\left|\psi_{\text {nor }}\left(k_{0} ; k\right)\right|^{2}, \\
\geq\left(\min _{y \in \mathcal{I}_{\gamma}}\left\{e^{-2 \delta \int_{y}^{y+t d / T_{0}} d x \bar{V}_{d}(x)}\right\}\right) \sum_{k=\bar{k}(t)-\gamma d / 2+1}^{\bar{k}(t)+\gamma d / 2-1}\left|\psi_{\text {nor }}\left(k_{0} ; k\right)\right|^{2},
\end{array}\right.
\end{gathered}
$$

where $\mathcal{I}_{\gamma}:=\{\bar{k}(t)-\gamma d / 2+1, \bar{k}(t)-\gamma d / 2+2, \ldots, \bar{k}(t)+\gamma d / 2-1\}$. In order for the summation in the definitions of $\Delta_{L}, \Delta_{C}, \Delta_{R}$ to be well defined, we need the constraints, $-d \gamma / 2+\bar{k}(t)-[-d / 2+\bar{k}(t)]=n_{1}, d \gamma / 2+\bar{k}(t)-1-$ $[-d \gamma / 2+\bar{k}(t)+1]=n_{2}, d \gamma / 2+\bar{k}(t)-[d / 2+\bar{k}(t)]=n_{3}$, for $n_{1}, n_{2}, n_{3} \in \mathbb{Z}$. A solution for $\gamma$ is

$$
\gamma=\gamma(m)=\frac{m-2}{d} \in(0,1), \quad \text { where } m=\left\{\begin{array}{l}
4,6,8, \ldots, d+2 \text { if } d=2,4,6, \ldots \\
3,5,7, \ldots, d+2 \text { if } d=3,5,7, \ldots
\end{array}\right.
$$

Before proceeding to bound the maximization and minimization in Eq. (F95), we bound the common factor term, which is approximately one. We find that 


$$
\begin{aligned}
\sum_{k=\bar{k}(t)-\gamma d / 2+1}^{\bar{k}(t)+\gamma d / 2-1}\left|\psi_{\mathrm{nor}}\left(k_{0} ; k\right)\right|^{2}= & \sum_{k \in \mathcal{S}_{d}\left(k_{0}+t d / T_{0}\right)}\left|\psi_{\mathrm{nor}}\left(k_{0} ; k-t d / T_{0}\right)\right|^{2}-\sum_{k=\bar{k}(t)-d / 2}^{\bar{k}(t)-\gamma d / 2}\left|\psi_{\mathrm{nor}}\left(k_{0} ; k\right)\right|^{2} \\
& -\sum_{k=\bar{k}(t)+\gamma d / 2}\left|\psi_{\mathrm{nor}}\left(k_{0} ; k\right)\right|^{2} \\
= & 1-\sum_{k=\bar{k}(t)-d / 2}\left|\psi_{\mathrm{nor}}\left(k_{0} ; k\right)\right|^{2}-\sum_{k=\bar{k}(t)+\gamma d / 2}^{\bar{k}(t)-\gamma d / 2}\left|\psi_{\mathrm{nor}}\left(k_{0} ; k\right)\right|^{2} .
\end{aligned}
$$

where in the last line we use Eq. (F41). The remaining two terms in Eq. (F97) have been bounded in Eqs. (F89) and (F92). Noting that $0 \leq e^{-2 \delta \int_{y}^{y+t d / T_{0}} d x \bar{V}_{d}(x)} \leq 1$, we can thus simplify Eq. (F95), to find that

$$
\Delta_{C}\left\{\begin{array}{l}
\leq \Delta_{C}^{(+)}, \\
\geq \Delta_{C}^{(-)}-\epsilon_{C},
\end{array}\right.
$$

where $\epsilon_{\mathrm{C}}$ is defined in the statement of the lemma and

$$
\begin{aligned}
\Delta_{C}^{(+)} & :=\max _{y \in \mathcal{I}_{\gamma}}\left\{e^{-2 \delta \int_{y}^{y+t d / T_{0}} d x \bar{V}_{d}(x)}\right\}, \\
\Delta_{C}^{(-)} & :=\min _{y \in \mathcal{I}_{\gamma}}\left\{e^{-2 \delta \int_{y}^{y+t d / T_{0}} d x \bar{V}_{d}(x)}\right\} .
\end{aligned}
$$

Our next aim is to find bounds on $t$, which determine whether $\Delta_{C}$ is approximately 1 or $e^{-2 \delta}$. First note that

$$
\begin{aligned}
\int_{y}^{y+t d / T_{0}} d x \bar{V}_{d}(x) & =\frac{2 \pi}{d} \int_{y}^{y+t d / T_{0}} d x \bar{V}_{0}\left(\frac{2 \pi}{d} x\right) \\
& =\int_{2 \pi y / d-x_{0}}^{2 \pi y / d+t 2 \pi / T_{0}-x_{0}} d x \bar{V}_{0}\left(x+x_{0}\right) .
\end{aligned}
$$

We can now use Eq. (F101) to find conditions for the time "before a tick is measured," namely conditions for which

$$
\int_{y}^{y+t d / T_{0}} d x \bar{V}_{d}(x) \leq 1-\left(1-\tilde{\epsilon}_{V}\right)=\tilde{\epsilon}_{V} .
$$

From Fig. 5 and Eq. (F101) we observe that Eq. (F102) is satisfied for all $y \in \mathcal{I}_{\gamma}$ if

$$
\begin{array}{r}
x_{v r}-2 \pi \leq \frac{2 \pi}{d} y-x_{0}, \\
\frac{2 \pi}{d} y+t \frac{2 \pi}{T_{0}}-x_{0} \leq x_{v l}=-x_{v r}
\end{array}
$$

for all $y \in \mathcal{I}_{\gamma}$. Or equivalently, if

$$
\frac{2 \pi}{d} \min _{y \in \mathcal{I}_{\gamma}}\{y\} \geq x_{v r}-2 \pi+x_{0},
$$

$$
\frac{2 \pi}{d} \max _{y \in \mathcal{I}_{\gamma}}\{y\} \leq x_{0}-\frac{2 \pi}{T_{0}} t-x_{v r},
$$

from which it follows that

$$
\begin{gathered}
x_{0} \leq 2 \pi-x_{v r}-\pi \gamma+\frac{2 \pi}{d}[\bar{k}(t)+1], \\
0 \leq t \frac{2 \pi}{T_{0}} \leq x_{0}-x_{v r}-\pi \gamma-\frac{2 \pi}{d}[\bar{k}(t)-1] .
\end{gathered}
$$

Thus recalling that $\bar{k}(t) \in[0,1]$, sufficient conditions on $x_{0}$ and $t$ for Eq. (F102) to be satisfied are

$$
\begin{aligned}
x_{0} & \leq 2 \pi-x_{v r}-\pi \gamma, \\
0 \leq t \frac{2 \pi}{T_{0}} & \leq x_{0}-x_{v r}-\pi \gamma .
\end{aligned}
$$

Similarly, we can work out conditions for the time "after a tick has occurred," i.e.,

$$
\int_{y}^{y+t d / T_{0}} d x \bar{V}_{d}(x) \geq 1-\tilde{\epsilon}_{V}
$$

for all $y \in \mathcal{I}_{\gamma}$ if

$$
\begin{gathered}
\frac{2 \pi}{d} y-x_{0} \leq-x_{v r}, \\
x_{v r} \leq \frac{2 \pi}{d} y+t \frac{2 \pi}{T_{0}}-x_{0}
\end{gathered}
$$




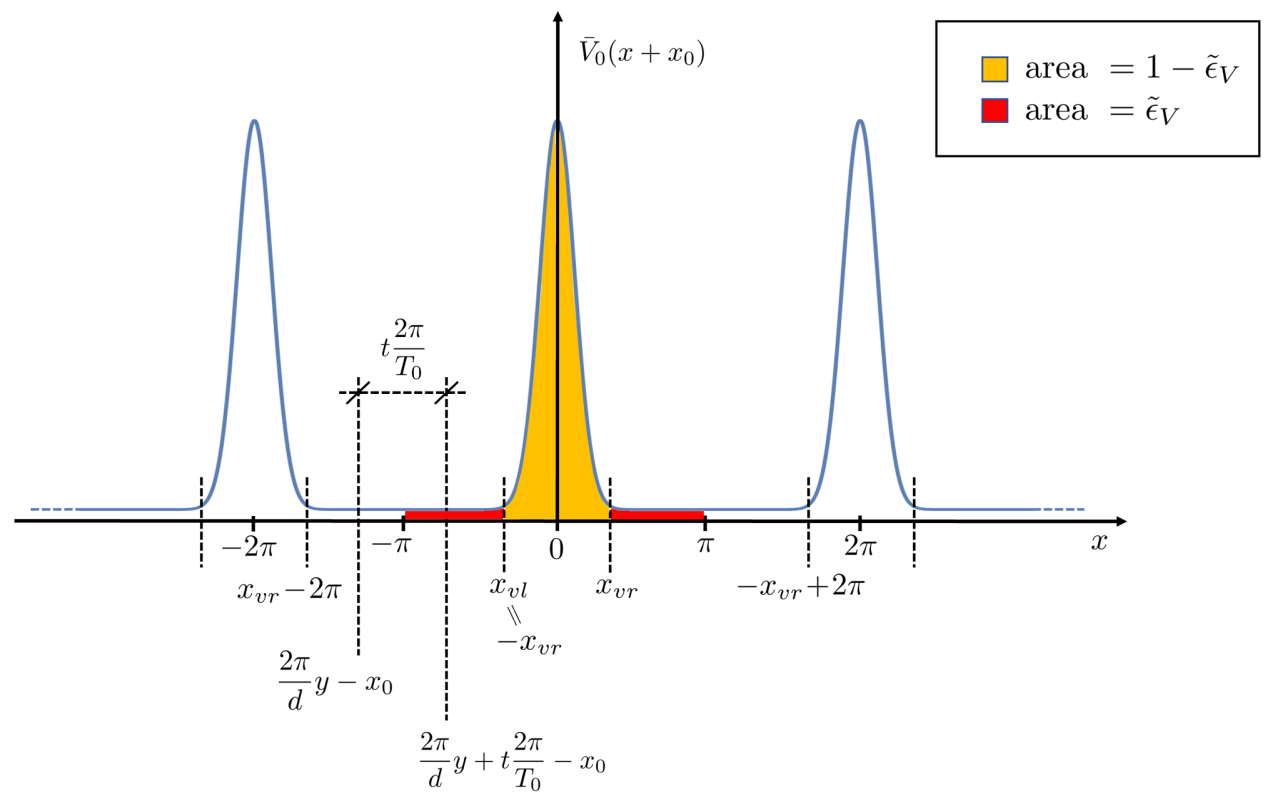

FIG. 5. Depiction of the periodic potential function $\bar{V}_{0}\left(x+x_{0}\right)$ with the parameters $x_{v l}, x_{v r}$, and $\tilde{\epsilon}_{V}$ introduced in Eq. (F13). Observe that the area under the potential $\bar{V}_{0}\left(x+x_{0}\right)$ (i.e., its integral) between $x=x_{v r}-2 \pi$ and $x=x_{v l}$ is $\tilde{\epsilon}_{V}$ due to symmetry. The quantities $(2 \pi / d) y-x_{0}$ and $\frac{2 \pi}{d} y+$ $t \frac{2 \pi}{T_{0}}-x_{0}$ are the lower and upper integration limits in Eq. (F101).

for all $y \in \mathcal{I}_{\gamma}$. Or equivalently, if

$$
\begin{gathered}
x_{v r}+\max _{y \in \mathcal{I}_{\gamma}}\{y\} \frac{2 \pi}{d} \leq x_{0}, \\
x_{v r}+\frac{2 \pi}{d} \max _{y \in \mathcal{I}_{\gamma}}\{-y\}+x_{0} \leq t \frac{2 \pi}{T_{0}},
\end{gathered}
$$

from which it follows

$$
\begin{gathered}
x_{v r}+\frac{2 \pi}{d}[\bar{k}(t)-1]+\pi \gamma \leq x_{0}, \\
x_{v r}-\frac{2 \pi}{d}[\bar{k}(t)+1]+\pi \gamma+x_{0} \leq t \frac{2 \pi}{T_{0}} .
\end{gathered}
$$

Thus recalling that $\bar{k}(t) \in[0,1]$, sufficient conditions on $x_{0}$ and $t$ for Eq. (F111) to be satisfied are

$$
\begin{aligned}
x_{v r}+\pi \gamma & \leq x_{0}, \\
x_{v r}+\pi \gamma+x_{0} & \leq t \frac{2 \pi}{T_{0}} .
\end{aligned}
$$

Thus from Eqs. (F94), (F109), (F110), and (F118), and recalling $\bar{V}_{0} \geq 0$, we conclude that if $x_{0}$ satisfies

$$
x_{v r}+\pi \gamma \leq x_{0} \leq 2 \pi-x_{v r}-\pi \gamma,
$$

then

$$
\begin{aligned}
e^{-2 \delta \tilde{\epsilon}_{V}} & \leq \Delta_{C}^{(-)}(t) \leq \Delta_{C}^{(+)}(t) \leq 1 \quad \text { if } \quad 0 \leq t \frac{2 \pi}{T_{0}} \\
& \leq x_{0}-x_{v r}-\pi \gamma
\end{aligned}
$$

\section{Calculating $\Delta(0)$ and $\Delta(1)$ and an explicit lower bound on $R_{1}$}

Define

$$
\begin{aligned}
t_{1} & :=\left(x_{0}-x_{v r}-\pi \gamma\right) \frac{T_{0}}{2 \pi}, \quad t_{2}:=\left(x_{0}+x_{v r}+\pi \gamma\right) \frac{T_{0}}{2 \pi}, \\
\Delta t & :=t_{2}-t_{1}=\left(x_{v r}+\pi \gamma\right) \frac{T_{0}}{\pi} .
\end{aligned}
$$

Furthermore, we want $t_{1}$ to be in the center of the range $\left[0, T_{0}\right]$. As such, we set $t_{1}=T_{0} / 2$, which taking into account the definition of $t_{1}$ in Eq. (F123) implies

$$
x_{0}=\pi+x_{v r}+\pi \gamma .
$$

Physically, Eq. (F124) means that the potential is peaked near the mid point $\pi$ and thus that the continuous measurements will occur approximately at a time $T_{0} / 2$. Note that Eq. (F124) is consistent with Eq. (F120) as long as $x_{v r}+\pi \gamma \leq \pi / 2$. As we see later, both $\gamma$ and $x_{v r}$ will be parametrized such that $\gamma, x_{v r} \rightarrow 0$ as $d \rightarrow \infty$ [56]. As such, this constraint will always be satisfied for sufficiently large $d[57]$. 
Lemma 23: $R_{1}$ satisfies the bound

$$
R_{1} \geq \frac{-\left[\left(2 T_{0} \epsilon_{0}\left(T_{0}\right)+\int_{0}^{T_{0}} d t\left|\epsilon_{C}(t)\right|\right)\left(t_{1} e^{-2 \delta \tilde{\epsilon}_{V}}+\Delta t\right)+\Delta t^{2}\right] / T_{0}^{2}+e^{-4 \delta \tilde{\epsilon}_{V}} / 4}{\epsilon_{4}+\epsilon_{3}+\frac{3}{4} e^{-\delta\left(1-\tilde{\epsilon}_{V}\right)}+\left(1-e^{-4 \delta \tilde{\epsilon}_{V}}\right) / 4+\frac{2}{T_{0}^{2}}\left(t_{2}-t_{1} e^{-2 \delta \tilde{\epsilon}_{V}}\right) \Delta t+\left(\frac{\Delta t}{T_{0}}\right)^{2}},
$$

where we define

$$
\begin{aligned}
& \epsilon_{3}:=\frac{2}{T_{0}^{2}} \int_{0}^{T_{0}} d t t\left[\Delta_{L}(t)+\Delta_{R}(t)\right] \\
& \epsilon_{4}:=\epsilon_{0}\left(T_{0}\right)+\frac{1}{T_{0}^{2}}\left(2 T_{0} \epsilon_{0}\left(T_{0}\right)+\int_{0}^{T_{0}} d t \epsilon_{C}(t)\right)\left(t_{1} e^{-2 \delta \tilde{\epsilon}_{V}}+\Delta t\right)+\epsilon_{1} / T_{0}^{2} .
\end{aligned}
$$

Proof. Using Lemma 21, followed by Lemma 22,

$$
\begin{aligned}
\int_{0}^{T_{0}} d t \operatorname{tr}[\rho(t)] & \geq \int_{0}^{T_{0}} d t\left[\Delta_{L}(t)+\Delta_{C}(t)+\Delta_{R}(t)-\epsilon_{0}\right] \\
& \geq-T_{0} \epsilon_{0}\left(T_{0}\right)-\int_{0}^{T_{0}} d t \epsilon_{\mathrm{C}}(t)+\int_{0}^{t_{1}} d t \Delta_{C}^{(-)}(t)+\int_{t_{1}}^{t_{2}} d t \Delta_{C}(t)+\int_{t_{2}}^{T_{0}} d t \Delta_{C}(t) \\
& \geq-T_{0} \epsilon_{0}\left(T_{0}\right)-\int_{0}^{T_{0}} d t \epsilon_{\mathrm{C}}(t)+t_{1} e^{-2 \delta \tilde{\epsilon}_{V}}+\int_{t_{1}}^{t_{2}} d t \Delta_{C}(t) .
\end{aligned}
$$

Thus,

$$
\begin{aligned}
& \left(\int_{0}^{T_{0}} d t \operatorname{tr}[\rho(t)]\right)^{2} \geq\left(-T_{0} \epsilon_{0}\left(T_{0}\right)-\int_{0}^{T_{0}} d t \epsilon_{\mathrm{C}}(t)+t_{1} e^{-2 \delta \tilde{\epsilon}_{V}}+\int_{t_{1}}^{t_{2}} d t \Delta_{C}(t)\right)^{2} \\
& \geq\left(-T_{0} \epsilon_{0}\left(T_{0}\right)-\int_{0}^{T_{0}} d t \epsilon_{\mathrm{C}}(t)\right)^{2}-\left(2 T_{0} \epsilon_{0}\left(T_{0}\right)+\int_{0}^{T_{0}} d t \epsilon_{\mathrm{C}}\right)\left(t_{1} e^{-2 \delta \tilde{\epsilon}_{V}}+\int_{t_{1}}^{t_{2}} d t \Delta_{C}(t)\right) \\
& +\left(t_{1} e^{-2 \delta \tilde{\epsilon}_{V}}+\int_{t_{1}}^{t_{2}} d t \Delta_{C}(t)\right)^{2} \\
& \geq-\left(2 T_{0} \epsilon_{0}\left(T_{0}\right)+\int_{0}^{T_{0}} d t \epsilon_{\mathrm{C}}(t)\right)\left(t_{1} e^{-2 \delta \tilde{\epsilon}_{V}}+\Delta t\right)+t_{1}^{2} e^{-4 \delta \tilde{\epsilon}_{V}}+2 e^{-2 \delta \tilde{\epsilon}_{V}} t_{1} \int_{t_{1}}^{t_{2}} d t \Delta_{C}(t) \\
& +\left(\int_{t_{1}}^{t_{2}} d t \Delta_{C}(t)\right)^{2} \\
& \geq-\left(2 T_{0} \epsilon_{0}\left(T_{0}\right)+\int_{0}^{T_{0}} d t \epsilon_{\mathrm{C}}(t)\right)\left(t_{1} e^{-2 \delta \tilde{\epsilon}_{V}}+\Delta t\right)+t_{1}^{2} e^{-4 \delta \tilde{\epsilon}_{V}}+2 e^{-2 \delta \tilde{\epsilon}_{V}} t_{1} \int_{t_{1}}^{t_{2}} d t \Delta_{C}(t)-\Delta t^{2} \\
& =\Delta^{2}(0) \text {, }
\end{aligned}
$$


where we use Definition (F123) and Lemma 22. Similarly,

$$
\begin{aligned}
\int_{0}^{T_{0}} d t t \operatorname{tr}[\rho(t)] & \leq \int_{0}^{T_{0}} d t\left[\Delta_{L}(t)+\Delta_{C}(t)+\Delta_{R}(t)+\epsilon_{0}\right] \\
& \leq \frac{T_{0}^{2}}{2}\left[\epsilon_{0}\left(T_{0}\right)+\epsilon_{3}\right]+\int_{0}^{t_{1}} d t t \Delta_{C}(t)+\int_{t_{1}}^{t_{2}} d t t \Delta_{C}(t)+\int_{t_{2}}^{T_{0}} d t t \Delta_{C}(t) \\
& \leq \frac{T_{0}^{2}}{2}\left[\epsilon_{0}\left(T_{0}\right)+\epsilon_{3}\right]+\frac{t_{1}^{2}}{2}+\int_{t_{1}}^{t_{2}} d t t \Delta_{C}(t)+e^{-\delta\left(1-\tilde{\epsilon}_{V}\right)} \frac{T_{0}^{2}-t_{2}^{2}}{2} \\
& =\Delta(1)
\end{aligned}
$$

where $\epsilon_{3}$ is defined in Eq. (F126). Setting $t_{1}=T_{0} / 2$ and simplifying Eq. (F43), we achieve

$$
\begin{aligned}
R_{1} \geq & \frac{\Delta^{2}(0)+\epsilon_{2}}{2 \Delta(1)-\Delta^{2}(0)+\epsilon_{1}} \\
= & \frac{\left[-\left(2 T_{0} \epsilon_{0}\left(T_{0}\right)+\int_{0}^{T_{0}} d t \epsilon_{\mathrm{C}}(t)\right)\left(t_{1} e^{-2 \delta \tilde{\epsilon}_{V}}+\Delta t\right)+T_{0}^{2} e^{-4 \delta \tilde{\epsilon}_{V}} / 4-\Delta t^{2}\right] / T_{0}^{2}+\left(2 e^{-2 \delta \tilde{\epsilon}_{V}} t_{1} \int_{t_{1}}^{t_{2}} d t \Delta \Delta_{C}(t)+\epsilon_{2}\right) / T_{0}^{2}}{\epsilon_{4}+\epsilon_{3}+\frac{3}{4} e^{-\delta\left(1-\tilde{\epsilon}_{V}\right)}+\left(1-e^{-4 \delta \tilde{\epsilon}_{V}}\right) / 4+\frac{2}{T_{0}^{2}} \int_{t_{1}}^{t_{2}} d t\left(t-t_{1} e^{-2 \delta \tilde{\epsilon}_{V}}\right) \Delta_{C}(t)+\left(\frac{\Delta t}{T_{0}}\right)^{2}} \\
\geq & \frac{-\left[\left(2 T_{0} \epsilon_{0}\left(T_{0}\right)+\int_{0}^{T_{0}} d t \epsilon_{\mathrm{C}}(t)\right)\left(t_{1} e^{-2 \delta \tilde{\epsilon}_{V}}+\Delta t\right)+\Delta t^{2}\right] / T_{0}^{2}+e^{-4 \delta \tilde{\epsilon}_{V} / 4}}{\epsilon_{4}+\epsilon_{3}+\frac{3}{4} e^{-\delta\left(1-\tilde{\epsilon}_{V}\right)}+\left(1-e^{-4 \delta \tilde{\epsilon}_{V}}\right) / 4+\frac{2}{T_{0}^{2}}\left(t_{2}-t_{1} e^{-2 \delta \tilde{\epsilon}_{V}}\right) \int_{t_{1}}^{t_{2}} d t \Delta \Delta_{C}(t)+\left(\frac{\Delta t}{T_{0}}\right)^{2}} \\
& \frac{-\left[\left(2 T_{0} \epsilon_{0}\left(T_{0}\right)+\int_{0}^{T_{0}} d t\left|\epsilon_{\mathrm{C}}(t)\right|\right)\left(t_{1} e^{-2 \delta \tilde{\epsilon}_{V}}+\Delta t\right)+\Delta t^{2}\right] / T_{0}^{2}+e^{-4 \delta \tilde{\epsilon}_{V} / 4}}{\epsilon_{4}+\epsilon_{3}+\frac{3}{4} e^{-\delta\left(1-\tilde{\epsilon}_{V}\right)}+\left(1-e^{-4 \delta \tilde{\epsilon}_{V}}\right) / 4+\frac{2}{T_{0}^{2}}\left(t_{2}-t_{1} e^{-2 \delta \tilde{\epsilon}_{V}}\right) \Delta t+\left(\frac{\Delta t}{T_{0}}\right)^{2}}
\end{aligned}
$$

where $\epsilon_{4}$ is defined in Eq. (F126).

\section{6. $R_{1}$ to leading order}

Lemma 24: Assume $\tilde{\epsilon}_{V} \rightarrow 0, \epsilon\left(T_{0}, d\right) \rightarrow 0, \delta \rightarrow \infty, a \rightarrow \infty$, and $\delta \tilde{\epsilon}_{V} \rightarrow 0$, in the limit $d \rightarrow \infty$, then using Big-O notation, $\mathcal{O}$, to leading order in $e^{-\delta}, \Delta t$, and $\delta \tilde{\epsilon}_{V} ; R_{1}$ is lower bounded by

$$
R_{1} \geq \frac{1 / 4+\mathcal{O}\left[\epsilon\left(T_{0}, d\right) d\right]+\mathcal{O}\left(\Delta t^{2}\right)}{\mathcal{O}\left[A^{2}\left(\frac{\sigma}{d^{\eta / 2}}+1\right) e^{-\frac{\pi}{2} d^{\eta}}\right]+\mathcal{O}\left[a^{2} d \epsilon\left(T_{0}, d\right)\right]+\mathcal{O}\left(a^{2} e^{-\delta}\right)+\mathcal{O}\left(\delta \tilde{\epsilon}_{V}\right)+3\left(\frac{\Delta t}{T_{0}}\right)^{2}}
$$

for all fixed constants $\eta>0$ and $\sigma, d$ satisfying $4 / \sigma<d^{\eta / 2} \leq d / \sigma$.

Proof. We start with a technical definition, which allows us to upper and lower bound $\gamma$ [defined in terms of the integer $m$ in Eq. (F79)] in terms of $\sigma$ and a power of $d^{\eta / 2}$, which will be crucial for the rest of the proof.

For $\eta>0$ with $4 / \sigma<d^{\eta / 2} \leq d / \sigma$, parametrize $m$ by

$$
m= \begin{cases}2\lfloor\bar{m}\rfloor & \text { if } d=2,4,6, \ldots \\ 2\lfloor\bar{m}\rfloor+1 & \text { if } d=3,5,7, \ldots\end{cases}
$$

where

$$
\bar{m}:= \begin{cases}\frac{d^{\eta / 2}}{2} \sigma+1 \in(3, d / 2+1] & \text { if } d=2,4,6, \ldots \\ \frac{d^{\eta / 2}}{2} \sigma+\frac{1}{2} \in(5 / 2,(d+1) / 2] & \text { if } d=3,5,7, \ldots\end{cases}
$$


Note the consistency of the domains of $\bar{m}$ and $m$ : the domain of $\bar{m}$ (which follows from the constraint $4 / \sigma<d^{\eta / 2} \leq$ $d / \sigma$ ) in Eq. (F148) implies [via Eq. (F147)] a range for $m$ of $m \in\{6,8,10, \ldots, d+2\}$ for $d=2,4,6, \ldots$, and $m \in$ $\{5,7,9, \ldots, d+2\}$ for $d=3,5,7 \ldots$, which is within the domain of $m$ defined in Eq. (F79).

From the bound $x-1 \leq\lfloor x\rfloor \leq x, x \in \mathbb{R}$, it follows $2 \bar{m}-2 \leq m \leq 2 \bar{m}$ and thus from Eqs. (F79), (F147), and (F148) it follows

$$
\gamma=\frac{m-2}{d} \leq \frac{2 \bar{m}-2}{d}=d^{\eta / 2} \frac{\sigma}{d} \text { for } d=2,4,6, \ldots
$$

and

$$
\gamma=\frac{m-2}{d} \geq \frac{2 \bar{m}-4}{d}=d^{\eta / 2} \frac{\sigma}{d}-\frac{2}{d} \quad \text { for } d=2,4,6, \ldots
$$

Similarly, we find that

$$
d^{\eta / 2} \frac{\sigma}{d}-\frac{1}{d} \leq \gamma \leq d^{\eta / 2} \frac{\sigma}{d} \text { for } d=1,3,5
$$

We now bound $\epsilon_{3}$ using Eqs. (F150) and (F151),

$$
\begin{gathered}
\epsilon_{3}=\frac{2}{T_{0}^{2}} \int_{0}^{T_{0}} d t t\left[\Delta_{L}(t)+\Delta_{R}(t)\right] \\
\leq\left(\frac{2 A}{T_{0}}\right)^{2} \int_{0}^{T_{0}} d t \frac{t e^{-\frac{2 \pi}{\sigma^{2}}[\gamma d / 2-\bar{k}(t)]^{2}}}{1-e^{-4 \pi|\gamma d / 2-\bar{k}(t)| / \sigma^{2}}} \leq\left(\frac{2 A}{T_{0}}\right)^{2} \int_{0}^{T_{0}} d t \frac{t e^{-\frac{2 \pi}{\sigma^{2}}(\gamma d / 2-1)^{2}}}{1-e^{-4 \pi(\gamma d / 2-1) / \sigma^{2}}}( \\
=2 A^{2} \frac{e^{-\frac{2 \pi}{\sigma^{2}}(\gamma d / 2-1)^{2}}}{1-e^{-4 \pi(\gamma d / 2-1) / \sigma^{2}}} \leq 2 A^{2} \frac{e^{-\frac{\pi}{2}\left(d^{\eta / 2}-4 / \sigma\right)^{2}}}{1-e^{-2 \pi\left(d^{\eta / 2}-4 / \sigma\right) / \sigma}}
\end{gathered}
$$

where the denominator of Eq. (F153) is finite since $d^{\eta / 2}-4 / \sigma>0$ and we use the observation that $1 /\left(1-e^{-x}\right)$ is monotonic decreasing in $x$ for $x>0$. In the $d \rightarrow \infty$ limit, there are a number of possibilities, depending on whether $d^{\eta / 2} / \sigma$ tends to infinity, zero, or a positive constant. By calculating these cases separately.

(1) If $\lim _{d \rightarrow \infty} d^{\eta / 2} / \sigma=\infty$ we find that

$$
\epsilon_{3}=\mathcal{O}\left(A^{2} \frac{\sigma}{d^{\eta / 2}} e^{-\frac{\pi}{2} d^{\eta}}\right)
$$

(2) If $\lim _{d \rightarrow \infty} d^{\eta / 2} / \sigma$ converges to a constant, we find that

$$
\epsilon_{3}=\mathcal{O}\left(A^{2} e^{-\frac{\pi}{2} d^{\eta}}\right)
$$

Thus recalling that Big-O notation captures the worse-case scenario (i.e., it is an upper bound in the limit), combining the conclusions from (1) and (2) above we find, for arbitrary $d^{\eta / 2} / \sigma$,

$$
\epsilon_{3}=\mathcal{O}\left[A^{2}\left(\frac{\sigma}{d^{\eta / 2}}+1\right) e^{-\frac{\pi}{2} d^{\eta}}\right]
$$

Similarly, we find that $\epsilon_{\mathrm{C}}$ defined in Eq. (F81) is of order

$$
\epsilon_{\mathrm{C}}=\mathcal{O}\left[A^{2}\left(\frac{\sigma}{d^{\eta / 2}}+1\right) e^{-\frac{\pi}{2} d^{\eta}}\right] .
$$

Recalling the definition of $\epsilon_{0}, \epsilon_{1}, \epsilon_{2}$, and $\epsilon_{4}$, we find that

$$
\epsilon_{0}=\mathcal{O}\left[d \epsilon\left(T_{0}, d\right)\right], \quad \epsilon_{1}=\mathcal{O}\left(a^{2} e^{-\delta}\right)+\mathcal{O}\left[a^{2} \epsilon\left(T_{0}, d\right)\right], \quad \epsilon_{4}=\mathcal{O}\left[d \epsilon\left(T_{0}, d\right)\right]+\mathcal{O}\left(e^{-\delta}\right)+\mathcal{O}\left(\epsilon_{\mathrm{C}}\right),
$$

thus concluding the proof. 
Corollary 9: Recall that $\Delta t / T_{0}=x_{v r} / \pi+\gamma$. Let the conditions in Lemma 24 be satisfied. In addition, if $x_{v r}$ is of higher order than $\gamma,\left[x_{v r}=o(\gamma)\right]$; and the order terms in Eq. (F146) are of higher order than $\gamma^{2},\left[i . e ., A^{2}\left(\sigma / d^{\eta / 2}+\right.\right.$ 1) $\left.e^{-(\pi / 2) d^{\eta}}=o(\gamma), a^{2} d \epsilon\left(T_{0}, d\right)=o(\gamma), a^{2} e^{-\delta}=o(\gamma), \delta \tilde{\epsilon}_{V}=o(\gamma)\right]$ it follows from Eqs. (F149) and (F151), that

$$
R_{1} \geq \frac{1}{12} \gamma^{-2}+o\left(\gamma^{-2}\right) \geq \frac{1}{12} \frac{d^{2-\eta}}{\sigma^{2}}+o\left(\frac{d^{2-\eta}}{\sigma^{2}}\right)
$$

where o is little-o notation.

Proof. Follows directly from Lemma 24 by keeping leading-order terms only.

\section{Showing that the limit requirements of Corollary 9 can be met}

The difficulty in proving the conditions given in Lemma 24 can be satisfied, and that $x_{v r}$ can be of higher order than $\gamma$, consists in finding a potential $\bar{V}_{0}$, which can be parametrized in terms of $d$ adequately. In this section, we demonstrate via a particular $\bar{V}_{0}$ that this can indeed be achieved. Recall that the potential $\bar{V}_{0}$, which we introduce here, is summarized in $\mathrm{Sec}$. F $2 \mathrm{~b}$ along with the particular parametrizations, which we pick in this section, as need be.

\section{a. Generic definition of $\bar{V}_{\mathbf{0}}$}

We start by defining $\bar{V}_{0}: \mathbb{R} \rightarrow \mathbb{R}$ appropriately. Let [58]

$$
\bar{V}_{0}(x):=\frac{1}{\delta d^{2}}+n A_{0} \sum_{p=-\infty}^{\infty} V_{B}\left[n\left(x-x_{0}-2 \pi p\right)\right],
$$

where $n \geq 1$ is a free parameter, $A_{0}$ a normalization constant such that

$$
\int_{0}^{2 \pi} \bar{V}_{0}(x) d x=1
$$

and

$$
V_{B}(x):=\operatorname{sinc}^{2 N}(x):=\left(\frac{\sin (\pi x)}{\pi x}\right)^{2 N},
$$

where $V_{B}(0)=1$ (defined by continuity in $x$ ) and $N \in \mathbb{N}_{>0}$ is another free parameter. In the following if statements about $\bar{V}_{0}$ or $V_{B}$ are made without mentioning either $n$ or $N$, it is to be understood that these properties hold for all $n \geq 1$ and $N \in \mathbb{N}_{>0}$.

We now show some useful properties of $\bar{V}_{0}$. Later we parameterize $n$ in terms of $d$, while $N$ will be a constant dependent on the small ( $d$-independent) parameter $\eta$ introduced in Lemma 24. Such parametrizations will eventually lead to the explicitly form of the potential summarized in Sec. F 2 b.

\section{b. Finding a value for $a$}

It will now become apparent why we included the term $1 /\left(\delta d^{2}\right)$ in the definition of the potential $\bar{V}_{0}$.

Corollary 10: The constant a defined in Eq. (F59) can be set equal to the following:

$$
a=\frac{T_{0}}{4 \pi} d^{2} .
$$

Proof. Since all the terms in the definition of $\bar{V}_{0}$ are non-negative, it follows $\min _{x \in[0,2 \pi]} \bar{V}_{0}(x) \geq 1 /\left(\delta d^{2}\right)$. Hence

$$
\frac{T_{0}}{4 \pi \delta} \frac{1}{\min _{x \in[0,2 \pi]} \bar{V}_{0}(x)} \leq \frac{T_{0}}{4 \pi} d^{2} .
$$

Equation (F163) is thus a direct consequence of Eq. (F59). 


\section{c. Properties of $\bar{V}_{\mathbf{0}}$}

First note that $\bar{V}_{0}$ is periodic with period $2 \pi$, and is infinitely differentiable. The first of these properties is clear from Eq. (F160) while the second will be demonstrated in Lemma 28. These two properties are required by definition in Ref. [8]. We now show some additional properties, specific to this particular choice of potential function $\bar{V}_{0}$.

Lemma 25: (Normalization.) $A_{0}$ is $n$ independent. In particular, it satisfies $A_{0}<\underline{A}_{0}$, where $\underline{A}_{0}$ is only a function of $N$.

Proof. Since for all $x \in[0,2 \pi]$,

$$
\sum_{p=-\infty}^{\infty}\left|V_{B}\left[n\left(x-x_{0}-2 \pi p\right)\right]\right| \leq 1+\sum_{p \in \mathbb{Z} \backslash\{0\}} \frac{1}{p^{2 N}}<\infty
$$

it follows from the Weierstrass M test (see Theorem 7.10 in Ref. [52]), that the sum in Eq. (F160) converges uniformly for all $x \in[0,2 \pi]$. We use this in the following to exchange summation and integration limits over a finite interval. Using Eq. (F161), we have

$$
\begin{aligned}
1 & =\int_{0}^{2 \pi} d x \bar{V}_{0}(x)=\int_{0}^{2 \pi} d x \bar{V}_{0}\left(x+x_{0}\right)=A_{0} n \int_{0}^{2 \pi} d x \sum_{p=-\infty}^{\infty} V_{B}[n(x-2 \pi p)]+\int_{0}^{2 \pi} \frac{d x}{\delta d^{2}} \\
& =A_{0} n \sum_{p=-\infty}^{\infty} \int_{0}^{2 \pi} d x V_{B}[n(x-2 \pi p)]+\frac{2 \pi}{\delta d^{2}}=A_{0} n \sum_{p=-\infty}^{\infty} \int_{-2 \pi p}^{2 \pi(1-p)} d x V_{B}(n x)+\frac{2 \pi}{\delta d^{2}}=A_{0} n \int_{-\infty}^{\infty} d x V_{B}(n x)+\frac{2 \pi}{\delta d^{2}} \\
& =A_{0} \int_{-\infty}^{\infty} d x V_{B}(x)+\frac{2 \pi}{\delta d^{2}}
\end{aligned}
$$

Hence,

$$
A_{0}=\frac{1-2 \pi /\left(\delta d^{2}\right)}{\int_{-\infty}^{\infty} d x V_{B}(x)}<\frac{1}{\int_{-\infty}^{\infty} d x V_{B}(x)}=: \underline{A}_{0},
$$

which is $n$ independent, since $V_{B}$ is, and well defined since $V_{B} \in L^{1}$ with a nonzero integral.

Lemma 26: (Technical lemma needed for Lemma 28.)

$$
\frac{d^{k}}{d x^{k}} \sum_{p=-\infty}^{\infty} V_{B}(x n-2 \pi n p)=\sum_{p=-\infty}^{\infty} \frac{d^{k}}{d x^{k}} V_{B}(x n-2 \pi n p),
$$

for all $k \in \mathbb{N}_{>0}$ and $x \in[0,2 \pi]$.

Proof. For $p= \pm 2, \pm 3, \pm 4, \ldots, x \in[0,2 \pi]$ and for all $k \in \mathbb{N}_{>0}$,

$$
\begin{aligned}
& \leq\left|\frac{d^{k}}{d x^{k}} V_{B}(x n-2 \pi n p)\right| \\
& \leq\left|\frac{n^{k}}{[\pi n(x-2 \pi p)]^{2 N}} \sum_{q=0}^{k}\left(\begin{array}{l}
k \\
q
\end{array}\right) \frac{(-2 N)(-2 N-1) \ldots(-2 N-q+1)}{n^{q}(x-2 \pi p)^{q}}\left[\frac{d^{k-q}}{d z^{k-q}} \sin ^{2 N}(\pi z)\right]_{z=n(x-2 \pi p)}\right|
\end{aligned}
$$




$$
\leq \frac{n^{k}}{\left(\pi^{2} n|p|\right)^{2 N}} \sum_{q=0}^{k}\left(\begin{array}{l}
k \\
q
\end{array}\right) \frac{|(-2 N)(-2 N-1) \ldots(-2 N-q+1)|}{n^{q}(2 \pi)^{q}} D_{k-q}=: M_{p}^{k},
$$

where we define $D_{k}:=\sup _{z \in \mathbb{R}}\left|d^{k} / d z^{k} \sin ^{2 N}(\pi z)\right|<\infty$ for $k \in \mathbb{N}_{\geq 0}$. Hence, since

$$
\sum_{p \in \mathbb{Z},|p| \geq 2} M_{p}^{k}<\infty
$$

from the Weierstrass M test (see Theorem 7.10 in Ref. [52]), uniform convergence of $\sum_{p=-\infty}^{\infty} d^{k} / d x^{k} V_{B}(x n-2 \pi n p)$ follows for all $k \in \mathbb{N}_{>0}$.

If $f_{n}: \mathbb{R} \rightarrow \mathbb{R}$ is a sequence of differentiable functions with domain $[a, b], a, b \in \mathbb{R}$ with derivatives $f_{n}^{\prime}$, and $\sum_{n=0}^{\infty} f_{n}^{\prime}$ converges uniformly on $[a, b]$, then if we define $f:=\sum_{n=0}^{\infty} f_{n}$ and $|f|<\infty$, it follows that $f^{\prime}=\sum_{n=0}^{\infty} f_{n}^{\prime}$, where $f^{\prime}$ is the derivative of $f$. This is a well-known theorem and can be found in, e.g., Theorem 7.17 [52]. Thus from Eq. (F174) for $k=1$, Eq. (F170) follows immediately for $k=1$. Proceeding inductively, we prove Eq. (F170) for all $k \in \mathbb{N}_{>0}$.

Lemma 27: (Technical lemma needed for Lemma 28.) Let $f$ denote the triangle function, namely

$$
f(x):= \begin{cases}0 & \text { if }|x| \geq 1 \\ 1-|x| & \text { otherwise }\end{cases}
$$

and let $\star$ denote Convolution. Then,

$$
f^{\star N}:=\underbrace{f \star f \star \ldots \star f}_{N \text {-times convolution product }} \in L^{1}
$$

is a continuous function, which is infinitely differentiable on the intervals $(-N,-N+1) \cup(-N+1,-N+2) \cup \ldots \cup$ $(N-1, N)$ and zero on the intervals $(-\infty,-N) \cup(N,+\infty)$ for all $N \in \mathbb{N}_{>0}$.

Proof. The proof is by induction. Let us start by defining the continuous functions $g_{n}: \mathbb{R} \rightarrow \mathbb{R}$ for $n \in \mathbb{N}_{>0}$,

$$
g_{n}(x):=P_{n, m}(x) \quad \text { if } m \leq x \leq m+1 \text { for } m \in \mathbb{Z},
$$

where

$$
\left.P_{n, m}(x)=0 \quad \text { if } m \in(-\infty,-n-1)\right] \cup[n,+\infty),
$$

and $\left\{P_{n, m}(x)\right\}$ are a set of real polynomials in $x$. Continuity of $g$ implies

$$
P_{n, m}(m+1)=P_{n, m+1}(m+1) \quad \text { for all } m \in \mathbb{Z}
$$

We denote the set of such functions $g_{n}$ by $\mathcal{S}_{n}$. Note that $f$ in Eq. (F175) belongs to $\mathcal{S}_{1}$. We start by showing that $\left(g_{1} \star\right.$ $\left.g_{n}\right)(y) \in \mathcal{S}_{n+1}$ for all $n \in \mathbb{N}_{>0}, g_{n} \in \mathcal{S}_{n}$ and $g_{1} \in \mathcal{S}_{1}$. For this we calculate $\left(g_{1} \star g_{n}\right)(y)$ for $y \in[q, q+1]$; for $q \in \mathbb{Z}$ and 
define $\delta_{y}:=y-q, 0 \leq \delta_{y} \leq 1$. We find that

$$
\begin{aligned}
\left(g_{1} \star g_{n}\right)(y) & =\int_{-\infty}^{\infty} d x g_{1}(x) g_{n}(x-y)=\sum_{m=-1,0} \int_{m}^{m+1} d x P_{1, m}(x) g_{n}(x-y) \\
& =\sum_{m=-1,0}\left(\int_{m}^{m+\delta_{y}} d x P_{1, m}(x) g_{n}\left(x-q-\delta_{y}\right)+\int_{m+\delta_{y}}^{m+1} d x P_{1, m}(x) g_{n}\left(x-q-\delta_{y}\right)\right) \\
& =\sum_{m=-1,0}\left(\int_{m}^{m-q+y} d x P_{1, m}(x) P_{n, m-q-1}(x-y)+\int_{m-q+y}^{m+1} d x P_{1, m}(x) P_{n, m-q}(x-y)\right) \\
& =: P^{\prime}(n, q ; y),
\end{aligned}
$$

where $P^{\prime}(n, q ; y)$ is a polynomial in $y$ with coefficient depending on $n$ and $q$. This follows by noting: (1) the integral of $P_{1, m}(x) P_{n, m-q-1}(x-y)$ and $P_{1, m}(x) P_{n, m-q}(x-y)$ with respect to $x$ are polynomials in both $x$ and $y$ (this is trivial to see by a formal power-law expansion). (2) The $x$ variable is then evaluated at a linear function in $y$, which leaves us with a polynomial in $y$. (3) These polynomials are then summed over the coefficients in $m$, which again is another polynomial in $y .\left(g_{1} \star g_{n}\right)(y)$ is continuous in $y \in \mathbb{R}$, since both $g_{1}$ and $g_{n}$ are continuous and the convolution of two continuous functions is continuous.

All that is left to show, to prove that $\left(g_{1} \star g_{n}\right)(y) \in \mathcal{S}_{n+1}$, is to show that $P^{\prime}(n, q ; y)=P_{n+1, q}(y)$ for some $P_{n+1, q}(y)$ obeying Eqs. (F177) and (F178). That $P^{\prime}(n, q ; y)$ obeys Eq. (F177) follows from the relationship between $y$ and $q$, namely $y \in[q, q+1]$. Equation (F178) is true for $P^{\prime}(n, q ; y)$ if

$$
P^{\prime}(n, q ; y)=0 \quad \text { if } q \in(-\infty,-n-2] \cup[n+1, \infty) .
$$

We now verify that Eq. (F184) is satisfied. $q \leq-n-2$ implies $m-q-1 \geq m+n+1 \geq n$ for $m=-1,0$. Thus it follows using Eq. (F178) that the term $P_{n, m-q-1}$ in line (F182) is zero for $q \leq-n-2$. Furthermore $q \leq-n-2$ implies $m-q \geq m+n+2 \geq n+1$ for $m=-1,0$. Thus it follows using Eq. (F178) that the term $P_{n, m-q}$ in Eq. (F182) is zero for $q \leq-n-2$. Hence $P^{\prime}(n, q ; y)=0$ for $q \leq-n-2$. Similarly we can verify that $P^{\prime}(n, q ; y)=0$ for $q \geq n+1$. This concludes the proof that $\left(g_{1} \star g_{n}\right)(y) \in \mathcal{S}_{n+1}$ for all $n \in \mathbb{N}_{>0}, g_{n} \in \mathcal{S}_{n}$, and $g_{1} \in \mathcal{S}_{1}$.

Now work inductively to conclude that $g_{1}^{\star N} \in \mathcal{S}_{N}$. Thus recalling that $f \in \mathcal{S}_{1}$ and noting that all functions in $\mathcal{S}_{N}$ satisfy the conditions on $f^{\star N}$ of the lemma, we conclude the proof.

Lemma 28: There exists $C_{0}=C_{0}(N)>0$, which is only a function of $N$, i.e., independent of $n, d$, and $k$, such that

$$
\max _{x \in[0,2 \pi]}\left|\frac{d^{k}}{d x^{k}} \bar{V}_{0}(x)\right| \leq n^{k+1} C_{0}^{k+1}, \quad \forall k \in \mathbb{N}_{\geq 0}, \quad \forall n \geq 1 .
$$

Proof. The proof will consist in writing the $k$ th derivative in Fourier space and using properties of the Fourier transform to calculate and upper bound the resultant expression.

We start by simplifying the expression for the derivative.

We start by noting that the Fourier transform of $V_{B} \in L^{1}$ can be computed via the convolution theorem,

$$
\mathcal{F}\left(V_{B}\right)(y)=\mathcal{F}\left[\left(\operatorname{sinc}^{2}\right)^{N}\right](y)=\underbrace{\left[\mathcal{F}\left(\operatorname{sinc}^{2}\right) \star \mathcal{F}\left(\operatorname{sinc}^{2}\right) \star \ldots \star \mathcal{F}\left(\operatorname{sinc}^{2}\right)\right]}_{N \text {-times convolution product }}(y),
$$

which is well defined, since the convolution theorem maps two $L^{1}$ functions to an $L^{1}$ function, and $\operatorname{sinc}^{2} \in L^{1}$. By direct calculation, we have that

$$
\mathcal{F}\left(\operatorname{sinc}^{2}\right)(y)= \begin{cases}0 & \text { if }|y| \geq 1, \\ 1-|y| & \text { otherwise }\end{cases}
$$

Thus, since the convolution of two finite support functions has finite support, we conclude by induction from Eq. (F186) that $\mathcal{F}\left(V_{B}\right)(y)$ has finite support. We denote the finite interval containing the support of $\mathcal{F}\left(V_{B}\right)(y)$ by $\left[y_{\min }, y_{\max }\right]$. Furthermore, note that $\mathcal{F}\left(\operatorname{sinc}^{2}\right)$ has a discontinuous derivative at three points on its domain $\left(0, y_{\min }, y_{\max }\right)$. When $\mathcal{F}\left(\operatorname{sinc}^{2}\right)$ 
is convoluted with itself, the resultant function might also have a finite set of points in its support interval at which it is not differentiable (see Lemma 27) [59]. Similarly due to Eq. (F186), $\mathcal{F}\left(V_{B}\right)(y)$ may have a finite set of points contained in $\left[y_{\min }, y_{\max }\right]$ at which the function is not differentiable [59]. From Lemma 27, we conclude that a set containing all such points is $\left\{y_{l}=-N-1+l\right\}_{l=1}^{2 N+1}$, where $y_{1}=y_{\min }, y_{2 N+1}=y_{\max }$. Finally, the last property of $\mathcal{F}\left(V_{B}\right)(y)$, which we need, is that since $\mathcal{F}\left(\operatorname{sinc}^{2}\right)(y)$ has finite right and left first and second derivatives in the interval $y \in\left[y_{\min }, y_{\max }\right]$, it follows that $\mathcal{F}\left(V_{B}\right)(y)$ also has finite left and right first and second derivatives in the interval $y \in\left[y_{\min }, y_{\max }\right]$ (see Lemma 27).

Using Lemma 26 and the change of variable $z=n x-2 \pi p$, we have for $k \in \mathbb{N}_{\geq 0}$

$$
\begin{aligned}
\max _{x \in[0,2 \pi]}\left|\frac{d^{k}}{d x^{k}} \bar{V}_{0}(x)\right| & =\max _{x \in[0,2 \pi]}\left|\frac{d^{k}}{d x^{k}} \bar{V}_{0}\left(x+x_{0}\right)\right| \\
& =n A_{0} \max _{x \in[0,2 \pi]}\left|\frac{d^{k}}{d x^{k}} \sum_{p=-\infty}^{\infty} V_{B}(x n-2 \pi n p)\right| \\
& =n A_{0} \max _{x \in[0,2 \pi]}\left|\sum_{p=-\infty}^{\infty} \frac{d^{k}}{d x^{k}} V_{B}(x n-2 \pi n p)\right| \\
& =n^{k+1} A_{0} \max _{x \in[0,2 \pi]}\left|\sum_{p=-\infty}^{\infty}\left[\frac{d^{k}}{d z^{k}} V_{B}(z)\right]_{z=n x-2 \pi n p}\right| \\
& =n^{k+1} A_{0} \max _{x \in[0,2 \pi]}\left|\sum_{p=-\infty}^{\infty}\left[\mathcal{F}^{-1}\left((2 \pi i y)^{k} \mathcal{F}\left(V_{B}\right)\right)(z)\right]_{z=n x-2 \pi n p}\right| \\
& =n^{k+1} A_{0} \max _{x \in[0,2 \pi]}\left|\sum_{p=-\infty}^{\infty} \int_{-\infty}^{\infty} d y(2 \pi i y)^{k} \mathcal{F}\left(V_{B}\right)(y) e^{-2 \pi i(n x-2 \pi n p) y}\right| \\
& \leq 2 n^{k+1} A_{0} \max _{x \in[0,2 \pi]} \sum_{p=0}^{\infty}\left|\int_{y_{\min }}^{y_{\max }} d y(2 \pi i y)^{k} \cos \left(4 \pi^{2} n p y\right) \mathcal{F}\left(V_{B}\right)(y) e^{-2 \pi i n x y}\right| .
\end{aligned}
$$

Note that the inverse Fourier transform is well defined on the domain $L^{2}$. Thus line (F192) is well justified since $(2 \pi i y)^{k} \mathcal{F}\left(V_{B}\right) \in L^{2} \forall k \in \mathbb{N}_{\geq 0}$, because $\mathcal{F}\left(V_{B}\right)$ has finite support.

For the term $p=0$ we have

$$
\begin{gathered}
\left|\int_{y_{\min }}^{y_{\max }} d y(2 \pi i y)^{k} \mathcal{F}\left(V_{B}\right)(y) e^{-2 \pi i n x y}\right| \leq\left(y_{\max }-y_{\min }\right)\left|2 \pi y_{\max }\right|^{k} \max _{x \in\left[y_{\max }, y_{\min }\right]}\left|\mathcal{F}\left(V_{B}\right)(x)\right| \\
\leq y_{\mathrm{M}}\left(2 \pi y_{\mathrm{M}}\right)^{k} \mathcal{F}_{\max }^{(0)}\left(V_{B}\right),
\end{gathered}
$$

where we introduce the notation $\max \left\{\left|y_{\min }\right|,\left|y_{\max }\right|, 1\right\}=N=: y_{\mathrm{M}}, \quad d^{k} / d y^{k} \mathcal{F}\left(V_{B}\right)(y)=: \mathcal{F}^{(k)}\left(V_{B}\right)(y), \quad$ and $\sup _{y \in\left(y_{1}, y_{2}\right) \cup\left(y_{2}, y_{3}\right) \cup \ldots \cup\left(y_{2 N}, y_{2 N+1}\right)}\left|\mathcal{F}^{(k)}\left(V_{B}\right)(y)\right|=: \mathcal{F}_{\max }^{(k)}\left(V_{B}\right)$. We now bound the other terms $p \neq 0$. In the following we denote, $\lim _{\varepsilon \rightarrow 0^{+}} \mathcal{F}^{(k)}\left(V_{B}\right)\left(y_{0} \pm \varepsilon\right)=: \mathcal{F}_{ \pm}^{(k)}\left(V_{B}\right)\left(y_{0}\right)$, and use primes to denote first derivatives with respect to $y$. We proceed by integration by parts twice, after splitting the integral up into sections in which $\mathcal{F}\left(V_{B}\right)(y)$ has continuous derivatives. We find that

$$
\begin{gathered}
\left|\int_{y_{\min }}^{y_{\max }} d y(2 \pi i y)^{k} \cos \left(4 \pi^{2} n p y\right) \mathcal{F}\left(V_{B}\right)(y) e^{-2 \pi i n x y}\right|=\left|\sum_{r=1, \ldots, 2 N} \int_{y_{r}}^{y_{r+1}} d y(2 \pi i y)^{k} \cos \left(4 \pi^{2} n p y\right) \mathcal{F}\left(V_{B}\right)(y) e^{-2 \pi i n x y}\right| \\
=\mid \underbrace{\mid \sum_{r=1, \ldots, 2 N}\left[(2 \pi i y)^{k} \frac{\sin \left(4 \pi^{2} n p y\right)}{4 \pi^{2} n p} \mathcal{F}\left(V_{B}\right)(y) e^{-2 \pi i n x y}\right]_{y_{r}}^{y_{r+1}}}_{=0 \text { since } \mathcal{F}\left(V_{B}\right)(y) \text { is continuos }}
\end{gathered}
$$




$$
\begin{aligned}
& -\sum_{r=1, \ldots, 2 N} \int_{y_{r}}^{y_{r+1}} d y \frac{\sin \left(4 \pi^{2} n p y\right)}{4 \pi^{2} n p}(2 \pi i y)^{k-1} e^{-2 \pi i n x y} 2 \pi i\left((k-2 \pi i y n x) \mathcal{F}\left(V_{B}\right)(y)+\mathcal{F}\left(V_{B}\right)(y)+y \mathcal{F}^{(1)}\left(V_{B}\right)(y)\right) \mid \\
& =\mid \lim _{\varepsilon \rightarrow 0^{+}} \sum_{r=1, \ldots, 2 N}\left[\frac{-\cos \left(4 \pi^{2} n p y\right)}{16 \pi^{4} n^{2} p^{2}}(2 \pi i y)^{k-1} e^{-2 \pi i n x y} 2 \pi i\left((k-2 \pi i y n x) \mathcal{F}\left(V_{B}\right)(y)+\mathcal{F}\left(V_{B}\right)(y)+y \mathcal{F}^{(1)}\left(V_{B}\right)(y)\right)\right]_{y_{r}+\varepsilon}^{y_{r+1}-\varepsilon} \\
& \text { May not be zero since } \mathcal{F}^{(1)}\left(V_{B}\right)(y) \text { may be discontinuos at points } y_{1}, y_{2} \ldots, y_{2 N+1} \\
& -\sum_{r=1, \ldots, 2 N} \int_{y_{r}}^{y_{r+1}} d y \frac{-\cos \left(4 \pi^{2} n p y\right)}{16 \pi^{4} n^{2} p^{2}}\left((2 \pi i y)^{k-1} e^{-2 \pi i n x y} 2 \pi i\left[(k-2 \pi i y n x) \mathcal{F}\left(V_{B}\right)(y)+\mathcal{F}\left(V_{B}\right)(y)+y \mathcal{F}^{(1)}\left(V_{B}\right)(y)\right]\right)^{\prime} \mid \\
& \leq \sum_{r=1, \ldots, 2 N+1}\left|\frac{-\cos \left(4 \pi^{2} n p y_{r}\right)}{16 \pi^{4} n^{2} p^{2}}\left(2 \pi i y_{r}\right)^{k-1} e^{-2 \pi i n x y_{r}} 2 \pi i y_{r}\left(\mathcal{F}_{-}^{(1)}\left(V_{B}\right)\left(y_{r}\right)-\mathcal{F}_{+}^{(1)}\left(V_{B}\right)\left(y_{r}\right)\right)\right| \\
& +\sum_{r=1, \ldots, 2 N} \frac{y_{r+1}-y_{r}}{16 \pi^{4} n^{2} p^{2}} \sup _{y \in\left(y_{r}, y_{r+1}\right)}\left|\left((2 \pi i y)^{k-1} e^{-2 \pi i n x y} 2 \pi i\left[(k-2 \pi i y n x) \mathcal{F}\left(V_{B}\right)(y)+\mathcal{F}\left(V_{B}\right)(y)+y \mathcal{F}^{(1)}\left(V_{B}\right)(y)\right]\right)^{\prime}\right| \\
& \leq \frac{1}{16 \pi^{4} n^{2} p^{2}}\left(\left(2 \pi y_{\mathrm{M}}\right)^{k-1} y_{\mathrm{M}} 4 \pi(2 N+1) \mathcal{F}_{\max }^{(1)}\left(V_{B}\right)\right. \\
& +\left(y_{\max }-y_{\min }\right) 2 \pi\left(2 \pi y_{\mathrm{M}}\right)^{k-1}\left[\left[(k-1)\left(2 \pi y_{\mathrm{M}}\right)^{-1}+n x\right]\left[\left(k+2 \pi y_{\mathrm{M}} n x+1\right) \mathcal{F}_{\max }\left(V_{B}\right)+y_{\mathrm{M}} \mathcal{F}_{\max }^{(1)}\left(V_{B}\right)\right] 2 \pi\right. \\
& \left.\left.+2 \pi n x \mathcal{F}_{\max }\left(V_{B}\right)+(k+2 \pi n x+2) \mathcal{F}_{\max }^{(1)}\left(V_{B}\right)+y_{\mathrm{M}} \mathcal{F}_{\max }^{(2)}\left(V_{B}\right)\right]\right) \text {. }
\end{aligned}
$$

Note the dependency on $n$ of the expression on the right-hand side of the inequality (F204). It is of the form ( $c_{00}+n c_{01}+$ $\left.n^{2} c_{02}\right) / n^{2}$, where $c_{00} \geq 0, c_{01} \geq 0, c_{02} \geq 0$ are $n$ independent. Thus since $n \in[1, \infty)$, the right-hand side of Eq. (F204) is upper bounded uniformly in $n$ by setting $n=1$. Similarly, the coefficients $c_{01} \geq 0, c_{02} \geq 0$ are upper bounded in $x \in[0,2 \pi]$, by setting $x=2 \pi$. We thus have for $|p| \in \mathbb{N}_{>0}$,

$$
\begin{aligned}
& \left|\int_{y_{\min }}^{y_{\max }} d y(2 \pi i y)^{k} \cos \left(4 \pi^{2} n p y\right) \mathcal{F}\left(V_{B}\right)(y) e^{-2 \pi i n x y}\right| \\
& \leq \frac{(2 \pi N)^{k-1}}{16 \pi^{4} p^{2}}\left(N 4 \pi(2 N+1) \mathcal{F}_{\max }^{(1)}\left(V_{B}\right)\right. \\
& +N 2 \pi\left[\left[(k+1)(2 \pi N)^{-1}+2 \pi\right]\left\{\left[k+(2 \pi)^{2} N+1\right] \mathcal{F}_{\max }\left(V_{B}\right)+N \mathcal{F}_{\max }^{(1)}\left(V_{B}\right)\right\} 2 \pi\right. \\
& \left.\left.\quad+(2 \pi)^{2} \mathcal{F}_{\max }\left(V_{B}\right)+\left[k+(2 \pi)^{2}+2\right] \mathcal{F}_{\max }^{(1)}\left(V_{B}\right)+N \mathcal{F}_{\max }^{(2)}\left(V_{B}\right)\right]\right),
\end{aligned}
$$


where we use $y_{\mathrm{M}}=N$. The right-hand side of the inequality (F205) can be written as

$$
\frac{(2 \pi N)^{k}}{16 \pi^{4} p^{2}}\left(c_{03}+c_{04} k+c_{05} k^{2}\right)
$$

with the coefficients $c_{03} \geq 0, c_{04} \geq 0, c_{05} \geq 0$ are $k, x$ and $p$ independent. Thus from Eqs. (F188), (F195), and (F205),

$$
\begin{gathered}
\max _{x \in[0,2 \pi]}\left|\frac{d^{k}}{d x^{k}} \bar{V}_{0}(x)\right|=\max _{x \in[0,2 \pi]}\left|\frac{d^{k}}{d x^{k}} \bar{V}_{0}\left(x+x_{0}\right)\right| \\
\leq 2 n^{k+1} A_{0}\left(N(2 \pi N)^{k} \mathcal{F}_{\max }^{(0)}\left(V_{B}\right)+\sum_{p \in \mathbb{Z} \backslash\{0\}} \frac{(2 \pi N)^{k}}{16 \pi^{4} p^{2}}\left(c_{03}+c_{04} k+c_{05} k^{2}\right)\right. \\
=2 n^{k+1} A_{0}(2 \pi N)^{k}\left[N \mathcal{F}_{\max }^{(0)}\left(V_{B}\right)+\frac{1}{16 \pi^{4}} \frac{2 \pi^{2}}{6}\left(c_{03}+c_{04} e^{\ln k}+c_{05} e^{2 \ln k}\right)\right] \\
\leq 2 n^{k+1} A_{0}(2 \pi N)^{k}\left[N \mathcal{F}_{\max }^{(0)}\left(V_{B}\right)+\frac{1}{48 \pi^{2}}\left(c_{03}+c_{04} e^{k}+c_{05} e^{2 k}\right)\right],
\end{gathered}
$$

for $k \in \mathbb{N}_{\geq 0}$. Thus recalling from Lemma 25 that $A_{0}$ is upper bounded by $\underline{A}_{0}$, which is only a function of $N$, from Eq. (F212), it follows that there exists a coefficient $C_{0}$, which depends only on $N$ such that Eq. (F185) holds.

\section{d. Determining a parametrization of $\boldsymbol{n}$ in terms of $d$ such that $\epsilon\left(T_{0}, d\right) \rightarrow 0$ quicker than any polynomial in $d$.}

Let us start by introducing the constraint

$$
\frac{d}{\bar{v} \sigma}=d^{\epsilon 5}, \quad \text { for some fixed constant } 0<\epsilon_{5} .
$$

It will also be useful to introduce the variable $\epsilon_{6}$, which is a function of $d$ and $\sigma$ via

$$
\sigma=d^{\epsilon_{6}}
$$

where $\epsilon_{6}$ uniformly bounded to the interval [60] $\epsilon_{5}<\epsilon_{6}<1$. We can rewrite Eq. (F32) as

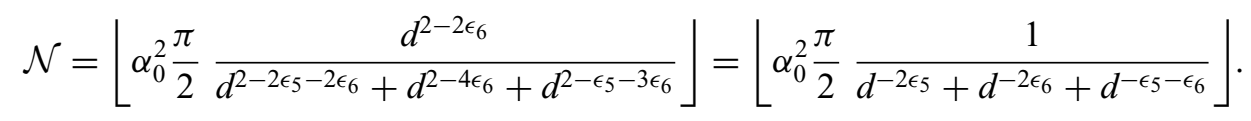

Thus recalling that $\alpha_{0} \in(0,1]$ is a fixed constant, it follows that $\mathcal{N}=\infty$ and $\bar{v} \geq 0$ in $d \rightarrow \infty$ limit. Hence Eq. (F37) are satisfied for sufficiently large $d$ and Eq. (F38) holds for the parameterizations considered in this proof in the limit $d \rightarrow \infty$. We can now rewrite Eq. (F38) as

$$
\begin{aligned}
\epsilon(t, d)= & |t| \frac{d}{T_{0}}\left[\mathcal{O}\left(\frac{\sigma^{3}}{\sigma d^{-\epsilon_{5}}+1}\right)^{1 / 2}+\mathcal{O}\left(\frac{d^{2}}{\sigma^{2}}+b\right)\right] \exp \left(-\frac{\pi}{4} \frac{\alpha_{0}^{2}}{\left(1+d^{\epsilon_{5}} / \sigma\right)^{2}} d^{2 \epsilon_{5}}\right) \\
& +\mathcal{O}\left(|t| \frac{d^{2}}{\sigma^{2}}+1\right) e^{-\frac{\pi}{4} \frac{d^{2}}{\sigma^{2}}}+\mathcal{O}\left(e^{-\frac{\pi}{2} \sigma^{2}}\right)
\end{aligned}
$$

thus since (as we soon show), $b$ grows at most polynomially in $d, \epsilon(t, d)$ decays quicker than any polynomial in $d$ in the limit $d \rightarrow \infty$. We now workout the implications of Eq. (F213). From Eq. (F31) and the relation $-i \delta \bar{V}_{0}(x)=V_{0}(x)$ stated 
in Eq. (F12), we have that $b$ is any non-negative number satisfying

$$
b \geq \sup _{k \in \mathbb{N}>0}\left(2 \max _{x \in[0,2 \pi]}\left|\delta \bar{V}_{0}^{(k-1)}(x)\right|\right)^{1 / k} .
$$

Thus from Lemma 28, it follows that

$$
\sup _{k \in \mathbb{N}_{>0}}\left(2 \max _{x \in[0,2 \pi]}\left|\delta \bar{V}_{0}^{(k-1)}(x)\right|\right)^{1 / k} \leq \sup _{k \in \mathbb{N}_{>0}}\left(2 \delta n^{k} C_{0}^{k}\right)^{1 / k}=2 \delta n C_{0},
$$

and hence we set $b=2 \delta n C_{0}$. Using the definition of $\bar{v}$ [Eq. (F33)], we have

$$
d^{\epsilon_{5}}=\frac{d}{\bar{v} \sigma}=\frac{d}{\sigma} \frac{\ln \left(\pi \alpha_{0} \sigma^{2}\right)}{\pi \alpha_{0} \kappa} \frac{1}{b}=\frac{d}{\sigma} \frac{\ln \left(\pi \alpha_{0} \sigma^{2}\right)}{2 \pi C_{0} \alpha_{0} \kappa} \frac{1}{\delta n},
$$

with $\kappa=0.792$ from which we find the constraint on $n$,

$$
n=\frac{\ln \left(\pi \alpha_{0} \sigma^{2}\right)}{2 \pi C_{0} \alpha_{0} \kappa} \frac{d^{1-\epsilon_{5}}}{\delta \sigma} .
$$

\section{e. Determining the constant $N$ and parametrization of $\delta$ in terms of $d$ such that conditions of Corollary 9 are satisfied}

From Eq. (F13) and using elementary properties of the potential $\bar{V}_{0}$ in Eq. (F160) [namely, integrates to unity over one period and is symmetric with respect to $x_{0}$, i.e., that $\bar{V}_{0}\left(x+x_{0}\right)=\bar{V}_{0}\left(-x+x_{0}\right)$ we find that

$$
\begin{aligned}
\tilde{\epsilon}_{V} & =1-\int_{-x_{v r}}^{x_{v r}} d x \bar{V}_{0}\left(x+x_{0}\right) \\
& =1-\left(\int_{-\pi}^{\pi} d x \bar{V}_{0}\left(x+x_{0}\right)-\int_{-\pi}^{-x_{v r}} d x \bar{V}_{0}\left(x+x_{0}\right)-\int_{x_{v r}}^{\pi} d x \bar{V}_{0}\left(x+x_{0}\right)\right) \\
& =1-\left(1-\int_{x_{v r}}^{\pi} d x \bar{V}_{0}\left(-x+x_{0}\right)-\int_{x_{v r}}^{\pi} d x \bar{V}_{0}\left(x+x_{0}\right)\right) \\
& =2 \int_{x_{v r}}^{\pi} d x \bar{V}_{0}\left(x+x_{0}\right) \\
& \leq 2\left(\pi-x_{v r}\right) \max _{x \in\left[x_{v r}, \pi\right]}\left\{\bar{V}_{0}\left(x+x_{0}\right)\right\} \\
& \leq 2 A_{0} n\left(\pi-x_{v r}\right) \sum_{p=-\infty}^{\infty} \max _{x \in\left[x_{v r}, \pi\right]}\left\{\left|V_{B}(n x-2 \pi p n)\right|\right\}+2 \frac{\left(\pi-x_{v r}\right)}{\delta d^{2}} \\
& \leq 2 \underline{A}_{0} n\left(\pi-x_{v r}\right) \sum_{p=-\infty}^{\infty} \max _{x \in\left[x_{v r}, \pi\right]}\left\{|\pi(n x-2 \pi p n)|^{-2 N}\right\}+2 \frac{\left(\pi-x_{v r}\right)}{\delta d^{2}} \\
& \leq 2 \underline{A}_{0} n\left(\pi-x_{v r}\right)\left[\left(n \pi x_{v r}\right)^{-2 N}+\left(2 \pi^{2} n\right)^{-2 N}\left(\sum_{p \in \mathbb{Z} \backslash\{0\}} p^{-2 N}\right)\right]+2 \frac{\left(\pi-x_{v r}\right)}{\delta d^{2}} \\
& \leq 2 \underline{A}_{0} \pi\left(\frac{n}{\left(n \pi x_{v r}\right)^{2 N}}+\frac{2 n \zeta(2 N)}{\left(2 \pi^{2} n\right)^{2 N}}\right)+\frac{2 \pi}{\delta d^{2}} \\
& \leq 2 \underline{A}_{0} \pi[1+\zeta(2 N)] \frac{n}{\left(n \pi x_{v r}\right)^{2 N}}+\frac{2 \pi}{\delta d^{2}} .
\end{aligned}
$$

Now recall Corollary 9. One of the conditions needed for this corollary to be satisfied is $x_{v r}=o(\gamma)$. Recalling the bound

$$
d^{\eta / 2} \frac{\sigma}{d}-\frac{2}{d} \leq \gamma \leq d^{\eta / 2} \frac{\sigma}{d}
$$


which is derived in Eqs. (F149) and (F151), we find that the following parametrization of $x_{v r}$ and $\delta$ allows us to achieve this scaling and the other conditions introduced in Corollary 9. Let

$$
\pi x_{v r}=d^{\epsilon 7} \frac{\sigma}{d}, \quad \delta=d^{\epsilon 8},
$$

for some fixed constants $0<\epsilon_{7}<\eta / 2,0<\epsilon_{8}$. We now show that indeed Eq. (F221) permits the existence of constants $N, \epsilon_{7}, \epsilon_{8}$ such that Eq. (F232) are satisfied.

Substituting expressions for $\pi x_{v r}$ and $n$ from Eqs. (F232), and (F220) into (F221), we find that

$$
\begin{aligned}
\delta \tilde{\epsilon}_{V} & \leq 2 \underline{A}_{0} \pi[1+\zeta(2 N)] \frac{\delta n}{\left(n \pi x_{v r}\right)^{2 N}}+\frac{2 \pi}{d^{2}} \\
& =2 \underline{A}_{0} \pi[1+\zeta(2 N)] \delta \frac{\ln \left(\pi \alpha_{0} \sigma^{2}\right)}{2 \pi C_{0} \alpha_{0} \kappa} \frac{d^{1-\epsilon_{5}}}{\delta \sigma}\left(\frac{2 \pi C_{0} \alpha_{0} \kappa}{\ln \left(\pi \sigma^{2}\right)} \frac{\delta \sigma}{d^{1-\epsilon_{5}}} \frac{1}{\epsilon^{\epsilon}} \frac{d}{\sigma}\right)^{2 N}+\frac{2 \pi}{d^{2}} \\
& =2 \underline{A}_{0} \pi[1+\zeta(2 N)]\left(\frac{2 \pi C_{0} \alpha_{0} \kappa}{\ln \left(\pi \alpha_{0} \sigma^{2}\right)}\right)^{2 N-1} \frac{d^{1-\epsilon_{5}+\left(\epsilon_{8}+\epsilon_{5}-\epsilon_{7}\right) 2 N}}{\sigma}+\frac{2 \pi}{d^{2}} .
\end{aligned}
$$

Now define a constant $\epsilon_{9}$ such that $0<\epsilon_{9}<\eta$, and impose the constraints $\epsilon_{7}>\epsilon_{5}+\epsilon_{8}$ and

$$
\frac{d^{1-\epsilon_{5}+\left(\epsilon_{8}+\epsilon_{5}-\epsilon_{7}\right) 2 N}}{\sigma} \leq d^{\epsilon_{9}}\left(\frac{\sigma}{d}\right)^{2} .
$$

Recalling $\sigma=d^{\epsilon_{6}}$, it follows from Eq. (F236) that

$$
\begin{aligned}
-3 \epsilon_{6}-\epsilon_{9}+2 & \leq-1+\epsilon_{5}+\underbrace{\left(\epsilon_{7}-\epsilon_{8}-\epsilon_{5}\right)}_{>0} 2 N \\
& \Longrightarrow N \geq \frac{-3 \epsilon_{6}-\epsilon_{9}-\epsilon_{5}+3}{2\left(\epsilon_{7}-\epsilon_{8}-\epsilon_{5}\right)} .
\end{aligned}
$$

Therefore, since $-\epsilon_{5}>-\epsilon_{6}$, we set the constant $N$ to be

$$
N:=\left\lceil\frac{3-4 \epsilon_{5}-\epsilon_{9}}{2\left(\epsilon_{7}-\epsilon_{8}-\epsilon_{5}\right)}\right\rceil>\frac{3-\epsilon_{5}-\epsilon_{9}-3 \epsilon_{6}}{2\left(\epsilon_{7}-\epsilon_{8}-\epsilon_{5}\right)},
$$

with $3-4 \epsilon_{5}-\epsilon_{9}>0$ and $\lceil\cdot\rceil$ denoting the ceiling function. Thus, from Eqs. (F221) and (F236), it follows that

$$
\delta \tilde{\epsilon}_{V} \leq 2 \underline{A}_{0} \pi[1+\zeta(2 N)]\left(\frac{2 \pi C_{0} \alpha_{0} \kappa}{\ln \left(\pi \alpha_{0} \sigma^{2}\right)}\right)^{2 N-1} d^{\epsilon_{9}}\left(\frac{\sigma}{d}\right)^{2}+\frac{2 \pi}{d^{2}} .
$$

Thus all the conditions for Corollary 9 are satisfied as long as all the constraints on the epsilons we introduce can simultaneously be satisfied. We show this in the proof of Theorem 6.

\section{Final theorem}

Theorem 6: Consider the setup described in Sec. F 1 for quasi-ideal clock and potential functions $\bar{V}_{0}$ introduced in Sec. $F 2$ with fixed constant $\alpha_{0} \in(0,1]$, and $k_{0}=0$. For all fixed constants $0<\eta \leq 1$, and $\sigma$ satisfying

$$
d^{\eta / 2} \leq \sigma<d,
$$

the clock accuracy $R_{1}$ is lower bounded by

$$
R_{1} \geq \frac{d^{2-\eta}}{\sigma^{2}}+o\left(\frac{d^{2-\eta}}{\sigma^{2}}\right)
$$

in the large d limit. 
Proof. The bound

$$
R_{1} \geq \frac{1}{12} \frac{d^{2-\eta}}{\sigma^{2}}+o\left(\frac{d^{2-\eta}}{\sigma^{2}}\right)
$$

follows directly from Corollary 9 and the results from Sec. 7, so long as the constraints on the epsilon terms can all be simultaneously satisfied. We check this here. The constraints introduced are as follows:

$$
\begin{gathered}
0<\epsilon_{5}<\epsilon_{6}<1, \quad 0<\epsilon_{7}<\frac{\eta}{2}, \quad 0<\epsilon_{8}, \quad \epsilon_{5} \\
+\epsilon_{8}<\epsilon_{7}, \quad 0<\epsilon_{9}<\eta, \quad 0<3-4 \epsilon_{5}-\epsilon_{9},
\end{gathered}
$$

and

$$
\frac{4}{\sigma}<d^{\eta / 2} \leq \frac{d}{\sigma}
$$

Let $\epsilon_{7}=\eta / 4, \epsilon_{5}=\epsilon_{8}=\eta / 16$, and $\epsilon_{9}=\eta / 2$. As long as

$$
\frac{\eta}{16}<\epsilon_{6}<1-\frac{\eta}{6}, \quad 0<\eta<4
$$

are satisfied, all constraints are met in Eq. (F244). Similarly, Eq. (F245) is satisfied if

$$
\frac{\ln 4}{\ln d}-\frac{\eta}{2}<\epsilon_{6} \leq 1-\frac{\eta}{2} .
$$

Hence Eqs. (F244) and (F246) are satisfied for sufficiently large $d$ if

$$
\eta<\epsilon_{6} \leq 1-\frac{\eta}{2}
$$

for $\eta>0$. Now make the substitution $\eta=\eta^{\prime} / 2$. Equation (F243) becomes

$R_{1} \geq \frac{d^{3 \eta^{\prime} / 4}}{12}\left[\frac{d^{2-\eta^{\prime}}}{\sigma^{2}}+o\left(\frac{d^{2-\eta^{\prime}}}{\sigma^{2}}\right)\right] \geq \frac{d^{2-\eta^{\prime}}}{\sigma^{2}}+o\left(\frac{d^{2-\eta^{\prime}}}{\sigma^{2}}\right)$,

with constraint

$$
d^{\frac{\eta^{\prime}}{2}}<\sigma \leq d^{1-\frac{\eta^{\prime}}{4}}, \quad \eta^{\prime}>0,
$$

where we use Eq. (F248) and recall $\sigma=d^{\epsilon_{6}}$. Finally, note that, without loss of generality, we can replace the upper bound on $\sigma$ in Eq. (F250) by $<d$. This is because of two observations: (1) By choosing $\eta^{\prime}$ arbitrarily close to zero, $\sigma$ is upper bounded by a number arbitrarily close to $d$. (2) The lower bound on $R_{1}$ in Eq. (F249) is monotonically decreasing with respect to $\eta^{\prime}$. To finalize the proof, we simply rename $\eta^{\prime}$ as $\eta$.
[1] Andrew D. Ludlow, Martin M. Boyd, Jun Ye, E. Peik, and P. O. Schmidt, Optical atomic clocks, Rev. Mod. Phys. 87, 637 (2015).

[2] Jonathan P. Dowling and Gerard J. Milburn, Quantum technology: The second quantum revolution, Philos. Trans. Royal Soc. A 361, 1655 (2003).

[3] Wolfgang Pauli, Handbuch der physik, Springer, Berlin 24, 83 (1933).

[4] Wolfgang Pauli, Encyclopedia of physics, Springer, Berlin 1, 60 (1958).

[5] Wolfgang Pauli, General Principles of Quantum Mechanics (Springer Science \& Business Media, Berlin, Germany, 2012).

[6] Sandra Ranković, Yeong-Cherng Liang, and Renato Renner, Quantum clocks and their synchronisation - the Alternate Ticks Game. ArXiv:1506.01373, 2015.

[7] Christopher A. Fuchs and Asher Peres, Quantum-state disturbance versus information gain: Uncertainty relations for quantum information, Phys. Rev. A 53, 2038 (1996).

[8] Mischa P. Woods, Ralph Silva, and Jonathan Oppenheim, Autonomous Quantum Machines and Finite-Sized Clocks. Annales Henri Poincaré, Oct (2018).

[9] Paul Erker, Mark T. Mitchison, Ralph Silva, Mischa P. Woods, Nicolas Brunner, and Marcus Huber, Autonomous Quantum Clocks: Does Thermodynamics Limit our Ability to Measure Time?, Phys. Rev. X 7, 031022 (2017).

[10] Helmut Salecker and Eugene P. Wigner, Quantum limitations of the measurement of space-time distances, Phys. Rev. 109, 571 (1958).

[11] Vladimir Bužek, Radoslav Derka, and Serge Massar, Optimal Quantum Clocks, Phys. Rev. Lett. 82, 2207 (1999).

[12] The word "clock" derives from the Medieval Latin "clocca," which means "bell." The hourly ringing of the bells may be regarded as an autonomous process.

[13] Sandra Stupar, Christian Klumpp, Nicolas Gisin, and Renato Renner, Performance of Stochastic Clocks in the Alternate Ticks Game. (2018). ArXiv:1806.08812.

[14] Gonzalo Muga, Rafael Sala Mayato, Ínigo Egusquiza, editors.Time in Quantum Mechanics Vol 1. Lecture Notes in Physics. Springer Berlin Heidelberg, (2007).

[15] Gonzalo Muga, Andreas Ruschhaupt, Adolfo del Campo, editors.Time in Quantum Mechanics Vol 2. Lecture Notes in Physics. Springer Berlin Heidelberg, (2010).

[16] We set $\hbar=1$, so that $i / \hbar[H, T]=i[H, T]=\mathbb{1}$.

[17] Thomas Pashby, Time and the foundations of quantum mechanics. PhD thesis, University of Pittsburgh, (2014).

[18] We note that this conclusion has been challenged and it has been argued that the relation $i[H, T]=\mathbb{1}$ can be satisfied for Hamiltonians $H$ with a semibounded spectrum if one considers operators with restricted domains of definition (see Ref. [17] for a discussion). Such restrictions, however, still correspond to unphysical assumptions, such as infinite potentials to keep a particle in a confined region.

[19] Asher Peres, Measurement of time by quantum clocks, Am. J. Phys. 48, 552 (1980).

[20] David Gross, Vincent Nesme, Holger Vogts, and Reinhard F. Werner, Index theory of one dimensional quantum walks and cellular automata, Commun. Math. Phys. 310, 419 (2012).

[21] At intermediate time intervals, the variance of the state with respect to the basis states $\left|\theta_{0}\right\rangle$ is as much as $\sqrt{d}$. 
[22] Mischa P. Woods, Autonomous ticking clocks from axiomatic principles. In preparation, (2020).

[23] More precisely, we could express such a clock via the convex combination of two maps, one that does have a zero tick probability for $\delta \rightarrow 0$ and one that does not. The second one would provide no time information, and thus would only worsen the performance of the clock.

[24] Paul Erker, The Quantum Hourglass. (2014). ETH Zürich.

[25] Göran Lindblad, On the generators of quantum dynamical semigroups, Commun. Math. Phys. 48, 119 (1976).

[26] John Preskill, Chapter 3. Foundations of Quantum Theory II: Measurement and Evolution. July 2015. Lecture notes available.

[27] While Eq. (12) does not define a dynamical semigroup $C T \rightarrow C T$, it is possible to do so, see Appendix 2 .

[28] More generally, the tick generators induce a mapping to some fixed state, but there is very little loss of generality setting the initial state to be the same, since only the first tick of the clock is affected, and every subsequent tick behaves as if the initial state is the fixed state.

[29] Maximilian A. Schlosshauer, Decoherence: and the Quantum-To-Classical Transition. The Frontiers Collection (Springer, Berlin Heidelberg, 2007).

[30] Igor Pikovski, Magdalena Zych, Fabio Costa, and Časlav Brukner, Universal decoherence due to gravitational time dilation, Nat. Phys. 11, 668 (2015).

[31] Wojciech Zurek, Quantum Darwinism. Nature Physics, 5, (2009).

[32] Equation (26) will be relaxed in the Appendix by replacing the "=" sign with " $\leq$." By doing so, we prove that our results for classical clocks hold under more general circumstances. The example of the maximally accurate classical clock in Sec. 1 satisfies Eq. (26).

[33] Paul Busch, The Time-Energy Uncertainty Relation (Springer Berlin Heidelberg, Berlin, Heidelberg, 2008).73

[34] Yakir Aharonov, Serge Massar, and Sandu Popescu, Measuring energy, estimating hamiltonians, and the timeenergy uncertainty relation, Phys. Rev. A 66, 052107 (2002).

[35] The saturation of the bound $\Delta E \Delta t=1 / 2$ by the quasi-ideal clock is up to an additive correction term, which decays faster than any polynomial in $d$.

[36] When the population $\left\langle\theta_{k}\left|\rho_{C}(t)\right| \theta_{k}\right\rangle$ reaches the position $d-$ 1 , rather than subsequently appearing at position 0 , it will be assigned to position $d$, and subsequently to position $d+1$, etc.

[37] Vittorio Giovannetti, Seth Lloyd, and Lorenzo Maccone, Advances in quantum metrology, Nat. Photonics 5, 222 (2011).

[38] Sebastian Deffner and Steve Campbell, Quantum speed limits: From heisenberg's uncertainty principle to optimal quantum control, J. Phys. A 50, 453001 (2017).

[39] Jacob D. Bekenstein, Generalized second law of thermodynamics in black-hole physics, Phys. Rev. D 9, 3292 (1974).

[40] Jacob D. Bekenstein and Marcelo Schiffer, Quantum limitations on the storage and transmission of information, Int. J. Mod. Phys. C 01, 355 (1990).

[41] E. Oelker, R. B. Hutson, C. J. Kennedy, L. Sonderhouse, T. Bothwell, A. Goban, D. Kedar, C. Sanner, J. M. Robinson, G. E. Marti, D. G. Matei, T. Legero, M. Giunta, R. Holzwarth, F. Riehle, U. Sterr, and J. Ye, Demonstration of $4.8 \times 10^{-} 17$ stability at $1 \mathrm{~s}$ for two independent optical clocks, Nat. Photonics 13, 714 (2019).

[42] As noted earlier, we require a state highly coherent over all $d$ degrees distinguishable states of the clock. In this sense, $d$ should be understood as an effective dimension, characterizing the space over which one has full control (corresponding to the logical qubits of a quantum computer).

[43] Note that current quantum computers are able to manipulate a larger number (50-100) of physical qubits, while the number of logical qubits is necessarily much smaller.

[44] Shishir Khandelwal, Maximilian P. E. Lock, and Mischa P. Woods, Universal quantum modifications to general relativistic time dilation in delocalised clocks, (2019).

[45] Charis Anastopoulos and Bei Lok Hu, Equivalence principle for quantum systems: Dephasing and phase shift of freefalling particles, Classical Quantum Gravity 35, 035011 (2018).

[46] Magdalena Zych, Fabio Costa, Igor Pikovski, and Časlav Brukner, Quantum interferometric visibility as a witness of general relativistic proper time, Nat. Commun. 2, 505 (2011).

[47] C. Budroni, G. Vitagliano, and Mischa P. Woods, Tickingclock performance enhanced by nonclassical temporal correlations, Phys. Rev. Research 3, 033051 (2021).

[48] For the axiomatic definitions of a dynamical semigroup see Definition 2 in Ref. [61]. In Ref. [25], Lindblad states these conditions on the adjoint map, which is equivalent to the conditions stated here since the clock is finite dimensional.

[49] Gilles Pütz, et al., In preparation, (2019).

[50] Pathological in the sense that such a function cannot be generated by the dynamics of finite-dimensional systems of bounded energy.

[51] Athanasios Papoulis and S. Unnikrishna Pillai, Probability, Random Variables, and Stochastic Processes (McGrawHill, Boston, 2002).

[52] Walter Rudin, Principles of Mathematical Analysis. International series in pure and applied mathematics. McGrawHill, 1976.

[53] Andrey Kolmogoroff, Über die analytischen methoden in der wahrscheinlichkeitsrechnung, Mathematische Annalen 104, 415 (1931).

[54] Note that $k$ will belong to a set of only $d$ consecutive integers so that $\left\{\left|\theta_{k}\right\rangle\right\}$ form a complete orthonormal basis without repetition.

[55] Rajendra Bhatia, Matrix analysis. Graduate Texts in Mathematics, 169. Springer-Verlag, New York, (1997).

[56] See Eqs. (F149), (F151), and (F232) for explicit parametrization.

[57] It is expected that one could actually weaken the constraint imposed on $x_{v r}$ and $\gamma$ if one choose a slightly different parametrization of $t_{1}$, say $t_{1}=T_{0} / 2-\left(x_{v r}+\pi \gamma\right) T_{0} / \pi$. But for simplicity, we do not do this here.

[58] One could also exchange the term $1 /\left(\delta d^{2}\right)$ with $1 /\left(\delta d^{K}\right)$ for some constant $K>0$ and the main result, Theorem 6 , would still go through.

[59] In fact, it is not differentiable at these points, but we do not need to prove this for our purposes.

[60] By "uniformly bounded," it is meant that $\epsilon_{5}$ $<\lim _{d \rightarrow \infty} \epsilon_{6}<1$.

[61] Andrzej Kossakowski, On quantum statistical mechanics of non-Hamiltonian systems, Rep. Math. Phys. 3, 247 (1972). 